\title{
Even Cycles in Graphs
}

Joseph G. Conlon

DEPARTMENT OF MATHEMATICS

UNIVERSITY OF MICHIGAN

ANN ARBOR, MICHIGAN 48109-1109

E-mail:conlon@umich.edu

Revised July 11, 2003

\begin{abstract}
Let $G$ be a 3-connected simple graph of minimum degree 4 on at least six vertices. The author proves the existence of an even cycle $C$ in $G$ such that $G-V(C)$ is connected and $G-E(C)$ is 2-connected. The result is related to previous results of Jackson, and Thomassen and Toft. Thomassen and Toft proved that $G$ contains an induced cycle $C$ such that both $G-V(C)$ and $G-E(C)$ is 2-connected. $G$ does not in general contain an even cycle such that $G-V(C)$ is 2-connected. (๑) 2004 Wiley Periodicals, Inc. J Graph Theory 45: 163-223, 2004
\end{abstract}

Keywords: even cycle; 3-connected; removable

\section{INTRODUCTION}

In this paper we shall be concerned with establishing the existence of an even cycle $C$ in a simple graph $G$. We want the cycle $C$ to have the property that if we remove the vertices $V(C)$ of $C$ or the edges $E(C)$ of $C$ from $G$, then the remaining graph is maximally connected. We prove the following theorem in this direction.

Theorem 1.1. Let $G$ be a connected simple graph of minimum degree 4. Then $G$ contains an even cycle $C$ such that $G-V(C)$ is non-empty and connected.

The graph with the least number of vertices which satisfies the conditions of Theorem 1.1 is the complete graph on five vertices, $K_{5}$, which is also

(c) 2004 Wiley Periodicals, Inc. 
3-connected. If we remove the vertices $V(C)$ of a cycle $C$ of order 4 from $K_{5}$, then the remaining graph is just the vertex graph and hence is connected. If we remove the edges $E(C)$ of $C$ then it is clear that $K_{5}-E(C)$ is connected but not 2-connected. In contrast to this example, we have the following.

Theorem 1.2. Let $G \neq K_{5}$ be a 3-connected simple graph of minimum degree 4 . Then $G$ contains an even cycle $C$ such that $G-V(C)$ is non-empty and connect$e d$, and $G-E(C)$ is 2-connected. Further, there is no triangle in $G$ consisting entirely of chords of $C$.

Consider the graph $G$ in Figure 1 below. Now $G$ has 11 vertices and its edges are denoted by both solid and dashed lines. It is clear that $G$ is 3-connected of minimum degree 4 . The even cycles in $G$ have orders 4,6 , and 8 . If a cycle $C$ has order 8, then $G-V(C)$ is disconnected. If $C$ has order 6 or 4 , then $G-V(C)$ is connected but not 2-connected. For any even cycle, $G-E(C)$ has a vertex of degree 2 , whence $G-E(C)$ is not 3-connected. The dashed edges in Figure 1 give a cycle of order 6 satisfying the conclusions of Theorem 1.2. Theorem 1.2 is proved using an induction argument. It appears to be necessary to include as part of the induction hypothesis that one cannot form a triangle from chords of $C$. See Lemma 3.6.

Theorem 1.2 should be compared to the following theorems of Thomassen and Toft [8].

Theorem 1.3. Let $G$ be a 3-connected simple graph of minimum degree 4. Then $G$ contains an induced cycle $C$ such that $G-V(C)$ and $G-E(C)$ are 2connected.

Theorem 1.4. Let $G$ be a 2-connected simple graph of minimum degree 4. Then $G$ contains an induced cycle $C$ such that $G-V(C)$ is connected and $G-E(C)$ is 2-connected.

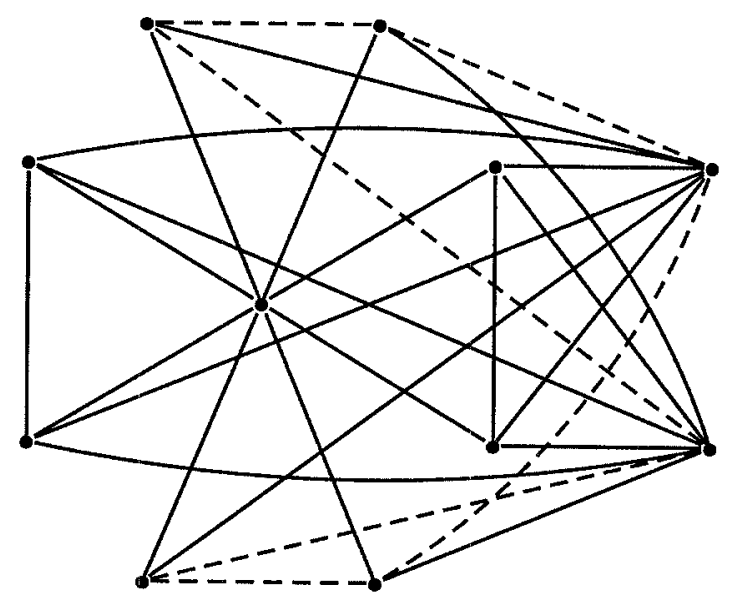

FIGURE 1. 
Theorem 1.4 answers in the affirmative a conjecture of Hobbs that if $G$ is a 2-connected simple graph of minimum degree 4, then $G$ contains a cycle $C$ such that $G-E(C)$ is 2-connected. Hobb's conjecture was first proved by Mader [7]. Later a stronger version of Mader's result was proved independently by Jackson [5]. More recently extensions of these results to matroids have been discussed by Lemos and Oxley [6].

In this paper we shall follow a similar strategy to that of [8] in order to prove Theorems 1.1 and 1.2. Thus in the proof of Theorem 1.1, we shall obtain the even cycle $C$ as the even cycle which maximises the number of vertices of the largest connected component of $G-V(C)$. For Theorem 1.2 we shall also need to maximise the number of vertices of the largest block of $G-V(C)$.

The problem of the existence of even cycles in graphs does not seem to have been studied much. Theorems 1.8 and 4.13 of [2], as well as [3], give some information on the existence of even cycles, but do not address the question of connectivity when the cycle is removed from the graph. The article of Frank [4] discusses the issue of connectivity when cycles are removed from graphs, but does not relate that to whether the cycle is even or odd. A classic text which discusses graph connectivity is the book of Tutte [9]. For existence of cycles in graphs, the book of Voss [10] gives a comprehensive survey of the literature until 1990. Throughout the paper we shall use the terminology of the recent book of Bollobas [1].

In general, in the paper, an arc of a cycle or a path in a graph is called even (odd) if it has an even (odd) number of vertices. Hence an even path has an odd number of edges.

\section{PROOF OF THEOREM 1.1}

We first identify the block decomposition of a graph which contains no even cycles.

Lemma 2.1. Suppose $G$ is a connected graph which contains no even cycles. Then in the block decomposition of $G$ each block is either a $K_{2}$ or an odd cycle.

Proof. If a block $B$ is not a $K_{2}$ then it must contain a cycle $C$ which is necessarily odd. Suppose $V(B) \neq V(C)$ and $v \in V(B)-V(C)$ is adjacent to a vertex $w \in V(C)$. Let $w^{\prime \prime} \neq w$ be another vertex of $C$. By Menger's theorem, there is a path in $B$ from $w^{\prime \prime}$ to $v$, which does not contain the vertex $w$. Let $w^{\prime}$ be the vertex of $C$ on this path closest to $v$ and $P\left(w^{\prime}, v\right)$ the corresponding path from $w^{\prime}$ to $v$. Since $C$ is odd it is clear, we can form an even cycle from one of the arcs $w^{\prime} w$ of $C$ joining $w^{\prime}$ to $w$, the edge $w v$ and the path $P\left(w^{\prime}, v\right)$. We have a contradiction, whence $V(B)=V(C)$. If $C$ has a chord then we can also form an even cycle with the chord and one of the arcs of $C$. We conclude that $B$ is precisely an odd cycle.

Corollary 2.1. A graph $G$ with minimum degree 3 contains an even cycle. 
Proof. Let $B$ be an endblock of the block decomposition of a connected component of $G$. If $B=K_{2}$ then $G$ has a vertex of degree 1. If $B$ is an odd cycle then $G$ has a vertex of degree 2 .

We begin the proof of Theorem 1.1. By Corollary 2.1, there is an even cycle $C^{\prime}$ such that $G-V\left(C^{\prime}\right)$ is non-empty. Let $C$ be an even cycle with the property that the largest connected component of $G-V(C)$ has the maximum number of vertices. Let $H$ be this maximal component. We wish to show that $H=$ $G-V(C)$. We shall prove this by contradiction, by assuming that $G-V(C)$ has a second connected component $H^{\prime}$.

Lemma 2.2. The component $H^{\prime}$ is a tree graph.

Proof. Now $H^{\prime}$ cannot contain an even cycle. Otherwise the graph on the vertices $V(H) \cup V(C)$ is connected and is disjoint from an even cycle in $G$. Suppose then that $H^{\prime}$ contains no even cycles and that $H$ attaches to a vertex $w \in V(C)$. Consider the endblocks in the block decomposition of $H^{\prime}$. If an endblock is an odd cycle $C^{\prime}$, then $C^{\prime}$ contains two vertices $a, b$, which are not cutvertices of $H^{\prime}$. Each of these vertices has degree at least 4 in $G$, whence $a$ attaches to a vertex $a^{\prime} \in V(C)$ with $a^{\prime} \neq w$, and similarly $b$ attaches to $b^{\prime} \in V(C)$ with $b^{\prime} \neq w$. It is possible for $a^{\prime}=b^{\prime}$. Let $a^{\prime} b^{\prime}$ be the arc of $C$, which does not contain $w$. Then one can form an even cycle with $a^{\prime} b^{\prime}$ and one of the arcs of $C^{\prime}$ joining $a$ to $b$. This lies outside the connected graph on the vertices $V(H) \cup\{w\}$, contradicting the definition of $H$.

Then we can assume that all endblocks in the block decomposition of $H^{\prime}$ are $K_{2}$ 's. Suppose that one of the blocks of $H^{\prime}$ is a cycle $C^{\prime}$. Then, two of the vertices of $C^{\prime}$, say $a$ and $b$, are cutvertices of $H^{\prime}$. There exist vertices $a^{\prime}, b^{\prime}$ of $H^{\prime}$ with the properties:

(a) $a^{\prime}, b^{\prime}$ have degree 1 in $H^{\prime}$;

(b) there is a path $P\left(a^{\prime}, a\right)$ in $H^{\prime}$ from $a^{\prime}$ to $a$ and similarly a path $P\left(b^{\prime}, b\right)$ from $b^{\prime}$ to $b$;

(c) the paths $P\left(a^{\prime}, a\right)$ and $P\left(b^{\prime}, b\right)$ do not intersect.

Evidently $a^{\prime}$ attaches to a vertex $a^{\prime \prime} \in V(C), a^{\prime \prime} \neq w$, and $b^{\prime}$ to $b^{\prime \prime} \in V(C)$, $b^{\prime \prime} \neq w$. Let $a^{\prime \prime} b^{\prime \prime}$ be the arc of $C$ joining $a^{\prime \prime}$ to $b^{\prime \prime}$, which does not contain $w$. Then one can form an even cycle from $a^{\prime \prime} b^{\prime \prime}, P\left(a^{\prime}, a\right), P\left(b^{\prime}, b\right)$ and one of the arcs of $C^{\prime}$ joining $a$ to $b$. This cycle lies outside the graph on the vertices $V(H) \cup\{w\}$, again contradicting the definition of $H$.

Lemma 2.3. Suppose $H^{\prime}$ is a tree graph and $w \in V(C)$. Then $G\left[V\left(H^{\prime}\right) \cup\right.$ $V(C)-\{w\}]$ contains an even cycle.

Proof. Assume first that $H^{\prime}$ is a single vertex. Then $H^{\prime}$ attaches to three vertices $a, b, c \in V(C)$ different from $w$. We can assume wlog that arc $a c$ of $C$ joining $a$ to $c$, which does not contain $w$, does include the vertex $b$. Let $a b$ and 
$b c$ be the arcs of $C$ joining $a$ to $b$ and $b$ to $c$, respectively, which are contained in $a c$. Consider now the three cycles: $C_{1}$ consisting of the arc $a c$ and $H^{\prime}, C_{2}$ consisting of the arc $a b$ and $H^{\prime}, C_{3}$ consisting of the arc $b c$ and $H^{\prime}$. It is easy to see that one of $C_{1}, C_{2}, C_{3}$ is even.

Hence we may assume $H^{\prime}$ contains two vertices $a, b$ of degree 1 in $H^{\prime}$. Let $a b$ be the path in $H^{\prime}$ joining $a$ to $b$. Then $a$ attaches to two vertices $a^{\prime}, a^{\prime \prime} \in V(C)$ different from $w$ and similarly $b$ to vertices $b^{\prime}, b^{\prime \prime} \in V(C)$ different from $w$. Let $a^{\prime} a^{\prime \prime}$ be the arc in $C$ joining $a^{\prime}$ to $a^{\prime \prime}$, which does not include $w$. We can assume $a^{\prime} a^{\prime \prime}$ has an even number of vertices. Otherwise the cycle consisting of $a^{\prime} a^{\prime \prime}$ and $a$ is even. We assume a similar situation with $b^{\prime}, b^{\prime \prime}$. Consider now the arc $a^{\prime} b^{\prime}$ in $C$ joining $a^{\prime}$ to $b^{\prime}$, which does not include $w$. If $a^{\prime} b^{\prime}$ is even then the arc $a^{\prime \prime} b^{\prime}$ joining $a^{\prime \prime}$ to $b^{\prime}$ and not including $w$ is odd. Conversely, if $a^{\prime} b^{\prime}$ is odd then $a^{\prime \prime} b^{\prime}$ is even. Hence we can form an even cycle with $a b$ and one of the $\operatorname{arcs} a^{\prime} b^{\prime}$ or $a^{\prime \prime} b^{\prime}$.

Proof of Theorem 1.1. This follows since Lemma 2.3 gives a contradiction to the definition of $H$.

\section{PROOF OF THEOREM 1.2-SETUP}

We begin by applying the maximization technique introduced in Section 2 to 2-connectedness.

Lemma 3.1. Let $G$ be a 3-connected graph and $C$ be an even cycle in $G$ with the property that $G-V(C)$ contains the largest block outside an even cycle. If $B^{*}$ is this largest block then every other block of $G-V(C)$ is either a $K_{2}$ or an odd cycle.

Proof. Assuming $G-V(C)$ is not necessarily connected, let $G^{*}$ denote the connected component of $G-V(C)$ containing $B^{*}$. The graph on the vertices $V\left(G^{*}\right) \cup V(C)$ is 2-connected. Hence if there is a block $B$ not in $G^{*}$, it must by Lemma 2.1 be either a $K_{2}$ or an odd cycle. If $B \subset G^{*}$, we can make a similar argument. In fact, there is a cutvertex $z$ such that $G^{*}=G_{1}^{*} \cup G_{2}^{*}$ with $V\left(G_{1}^{*}\right) \cap$ $V\left(G_{2}^{*}\right)=\{z\}$ and $B^{*} \subset G_{1}^{*}, B \subset G_{2}^{*}$. Since the graph on the vertices $V(C) \cup$ $V\left(G_{1}^{*}\right)-\{z\}$ is 2-connected, it follows again from Lemma 2.1 that $B$ is either a $K_{2}$ or an odd cycle.

We now do a further maximization of the cycle $C$ of Lemma 3.1.

Lemma 3.2. Suppose $G$ is a 3-connected graph of minimum degree 4. Let $C$ be an even cycle with the properties:

(a) $G-V(C)$ contains a largest block $B^{*}$ outside an even cycle.

(b) The connected component of $G-V(C)$ containing $B^{*}$ is the largest connected component containing $B^{*}$ which lies outside an even cycle.

Then $G-V(C)$ is connected. 
Proof. Suppose $H^{\prime}$ is a second connected component of $G-V(C)$. Then from the arguments of Lemmas 2.2 and 2.3, we obtain a contradiction.

Remark 1. Note that the graph $G-V(C)$ of Lemma 3.2 could be a vertex graph.

Proposition 3.1. Let $N>5$ be an integer and assume Theorem 1.2 holds for all graphs $G^{\prime}$ with $\left|V\left(G^{\prime}\right)\right|=N-2$. Let $G$ be a graph satisfying the conditions of Theorem 1.2 with $|V(G)|=N$. Suppose $C$ is an even cycle in $G$ satisfying (a) and (b) of Lemma 3.2. Then $G-V(C)$ is not a vertex graph. If $G-V(C)$ is 2connected, then there is an even cycle $C^{\prime}$ in $G$ such that $G-V\left(C^{\prime}\right)$ is non-empty and connected, and $G-E\left(C^{\prime}\right)$ is 2-connected. Further, a triangle cannot be formed entirely from chords of $C^{\prime}$.

We prove Proposition 3.1 in a series of lemmas.

Lemma 3.3. Let $C$ be the even cycle of Lemma 3.2 and suppose $C$ has order larger than 4. Then $C$ has at most one chord.

Proof. Let $x, y$ be two vertices of $C$, which are non-adjacent on the cycle $C$ but for which the edge $x y$ exists. Let $\operatorname{arc}(x, y)$ denote a path from $x$ to $y$ on $C$. Since $C$ is connected to $G-V(C)$ by at least three vertices, one can form a cycle $C^{\prime}$ from the edge $x y$ and one of the $\operatorname{arcs}, \operatorname{arc}(x, y)$, such that $G-V\left(C^{\prime}\right)$ is connected. If $\operatorname{arc}(x, y)$ is even we have a contradiction to (b) of Lemma 3.2. We conclude that $\operatorname{arc}(x, y)$ is odd.

Suppose now there is a third vertex $z$ of $C$, non-adjacent to $x$ on $C$, for which the edge $x z$ also exists. Then an $\operatorname{arc}, \operatorname{arc}(x, z)$, must be odd. Let $\operatorname{arc}(y, z)$ be the path from $y$ to $z$ on $C$, which does not include $x$. We may then choose $\operatorname{arc}(x, y)$ and $\operatorname{arc}(x, z)$ so that $C$ is the union of $\operatorname{arc}(x, y), \operatorname{arc}(y, z)$ and $\operatorname{arc}(x, z)$. Evidently the cycle $C^{\prime}$ consisting of $\operatorname{arc}(y, z)$ and the edges $x y$ and $x z$ is even. It follows again from (b) of Lemma 3.2 that no vertex of $V(C)-V\left(C^{\prime}\right)$ attaches to $G-V(C)$. Hence at least three vertices of $C^{\prime}$ attach to $G-V(C)$.

Let $w$ be an internal vertex of $\operatorname{arc}(x, y)$, i.e., $w \neq x, y$, and $v$ be a vertex of $\operatorname{arc}(x, z)$, non-adjacent to $w$ on $C$, such that the edge $w v$ exists. As before, $\operatorname{arc}(w, v)$ must be odd. Suppose that $v \neq z$ and let $C^{\prime \prime}$ be the cycle consisting of $\operatorname{arc}(w, y) \subset \operatorname{arc}(x, y), \operatorname{arc}(x, v) \subset \operatorname{arc}(x, z)$, together with the edges $x y$ and $w v$. Then $C^{\prime \prime}$ is even and $V\left(C^{\prime \prime}\right) \cap V\left(C^{\prime}\right)=\{x, y\}$. Since three vertices of $C^{\prime}$ attach to $G-V(C)$, we obtain a contradiction to (b) of Lemma 3.2. We get a similar contradiction if $v \neq x$. We conclude that $w$ does not attach to any vertex of $\operatorname{arc}(x, z)$.

Suppose next that $v$ is a vertex of $\operatorname{arc}(y, z)$, non-adjacent to $w$ on $C$, such that the edge $w v$ exists. Let $C_{1}^{\prime \prime}$ be the cycle consisting of $\operatorname{arc}(w, x) \subset \operatorname{arc}(x, y)$, $\operatorname{arc}(y, v) \subset \operatorname{arc}(y, z)$, together with the edges $x y$ and $w v$. Let $C_{2}^{\prime \prime}$ be the cycle consisting of $\operatorname{arc}(w, x) \subset \operatorname{arc}(x, y), \operatorname{arc}(v, z) \subset \operatorname{arc}(y, z)$, together with the edges $x z$ and $w v$. It is easy to see as before that $C_{1}^{\prime \prime}$ and $C_{2}^{\prime \prime}$ are even cycles. Further, one has $V\left(C^{\prime}\right) \cap V\left(C_{1}^{\prime \prime}\right) \cap V\left(C_{2}^{\prime \prime}\right)=\{x, v\}$. Hence for either $i=1$ or $2, V\left(C^{\prime}\right)-V\left(C_{i}^{\prime \prime}\right)$ has a vertex which attaches to $G-V(C)$. Again we obtain a contradiction. 
Suppose finally that $v$ is a vertex of $\operatorname{arc}(x, y)$ such that $v$ is non-adjacent on $C$ to $w$ and the edge $w v$ exists. Assume $v$ is the nearest of $w$ and $v$ to $y$ on $C$. Let $C^{\prime \prime}$ be the cycle consisting of $\operatorname{arc}(x, w) \subset \operatorname{arc}(x, y), \operatorname{arc}(v, y) \subset \operatorname{arc}(x, y)$, together with the edges $x y$ and $w v$. Then $C^{\prime \prime}$ is even and $V\left(C^{\prime \prime}\right) \cap V\left(C^{\prime}\right)=\{x, y\}$. We have a contradiction as previously.

We conclude then that $w$ has degree 2 in the graph on $V(C)$. Since we have already seen that $w$ cannot attach to $G-V(C)$, we have a contradiction. Hence every vertex of $C$ has at most one chord, whence every vertex of $C$ attaches to $G-V(C)$.

Suppose now that there are four distinct vertices of $C, x, y, w, v$ such that $x$ and $y$ are non-adjacent on $C$ but the edge $x y$ exists and similarly $w$ and $v$ are non-adjacent but the edge $w v$ exists. Suppose first that $w$ and $v$ are on different $\operatorname{arcs}, \operatorname{arc}(x, y)$. Since $C$ has order larger than 4 , we can assume wlog that $w$ is not adjacent on $C$ to $y$. Let $C^{\prime}$ be the cycle consisting of $\operatorname{arc}(x, w) \subset \operatorname{arc}(x, y)$, $\operatorname{arc}(v, y) \subset \operatorname{arc}(x, y)$, together with the edges $x y$ and $w v$. Since $\operatorname{arc}(x, y)$ and $\operatorname{arc}(w, v)$ are odd, it follows that $C^{\prime}$ is an even cycle. Since $w$ is not adjacent on $C$ to $y$, one also has that $V(C)-V\left(C^{\prime}\right)$ is non-empty. We have already observed that every vertex of $C$ attaches to $G-V(C)$, whence we obtain a contradiction. Next assume $w, v$ are on the same $\operatorname{arc}, \operatorname{arc}(x, y)$, with $w$ closest to $x$ on $C$. Let $C^{\prime}$ be the cycle consisting of $\operatorname{arc}(x, w) \subset \operatorname{arc}(x, y), \operatorname{arc}(v, y) \subset \operatorname{arc}(x, y)$, together with the edges $x y$ and $v w$. Then $C^{\prime}$ is an even cycle and $V(C)-V\left(C^{\prime}\right)$ is non-empty. We obtain a contradiction as before.

Corollary 3.1. Let $C$ be the even cycle of Lemma 3.2 and suppose $C$ has order larger than 4. Then $G-V(C)$ has at least two vertices.

Proof. By Lemma 3.3, there is a vertex of $C$, which has degree 2 in the graph on $V(C)$. Since this vertex has minimum degree $4, G-V(C)$ has at least two vertices.

Lemma 3.4. Let $C$ be the even cycle of Lemma 3.2 and suppose $C$ has order larger than 4. If $G-V(C)$ is 2-connected, then there is an even cycle $C^{\prime}$ in $G$ such that $G-V\left(C^{\prime}\right)$ is non-empty and connected, and $G-E\left(C^{\prime}\right)$ is 2-connected. Further, one cannot form a triangle entirely from chords of $C^{\prime}$.

Proof. Suppose first that $C$ has no chords. Then every vertex of $C$ attaches to two vertices of $G-V(C)$. Since $G-V(C)$ is 2-connected, it follows that $G-E(C)$ is 2-connected. Assume next that $C$ has exactly one chord with vertices $x, y \in V(C)$. We may also assume as before that $\operatorname{arc}(x, y)$ is odd. Now $x$ attaches to a vertex $x^{\prime} \in G-V(C)$ and $y$ to a vertex $y^{\prime} \in G-V(C)$. If $x^{\prime} \neq y^{\prime}$ then it is clear that $G-E(C)$ is 2-connected. Suppose now $x^{\prime}=y^{\prime}$ and let $C^{\prime}$ be the cycle consisting of the smaller of the $\operatorname{arcs}, \operatorname{arc}(x, y)$, and the vertex $x^{\prime}$. Evidently $C^{\prime}$ is even and $G-V\left(C^{\prime}\right)$ is connected. Consider now $G-E\left(C^{\prime}\right)$. We need to show that there are two disjoint paths in $G-E\left(C^{\prime}\right)$ linking $x$ to $G-V(C)$, and 
similarly with $y$. Since $C$ has at least six vertices we have $|V(C)-V(\operatorname{arc}(x, y))|$ $\geq 3$. Let $w$ be the vertex on $C-\operatorname{arc}(x, y)$ adjacent to $x$ and $v$ be the vertex adjacent to $y$, whence $w \neq v$. Then $w$ attaches to a vertex $w^{\prime}$ of $G-V(C)$ and $v$ to a vertex $v^{\prime}$ of $G-V(C)$ with $v^{\prime} \neq w^{\prime}$. Hence $x$ can be connected to $G-V(C)$ by the disjoint paths $x w w^{\prime}$ and $x y v v^{\prime}$ in $G-E\left(C^{\prime}\right)$. Since $C$ has just one chord, it follows that one cannot form a triangle from chords of $C^{\prime}$.

Lemma 3.5. Let $C$ be the even cycle of Lemma 3.2 and suppose $C$ has order 4 with either no chords or 2 chords. If $|V(G)| \geq 6$ and $G-V(C)$ is 2-connected, then there is a cycle $C^{\prime}$ of order 4 in $G$ such that $G-V\left(C^{\prime}\right)$ is non-empty and connected, and $G-E\left(C^{\prime}\right)$ is 2-connected.

Proof. If $C$ has no chords we can argue as in Lemma 3.4 to conclude $G-E(C)$ is 2-connected. Suppose now $C$ has two chords in which case the graph on $V(C)$ is $K_{4}$. Consider first the case when $G-V(C)=K_{2}$ with vertices $A$ and $B$. Now $A$ attaches to at least three vertices of $C$ so let us denote by $a$ the vertex of $C$ to which $A$ may not attach. Similarly denote by $b$ the vertex of $C$ to which $B$ may not attach. Observe that both $a$ and $b$ must attach to one of the vertices $A$ or $B$. It follows easily that $G-E(C)$ is 2-connected.

Next consider the case when $G-V(C)$ has at least three vertices. Since $G$ is 3-connected and the graph on $V(C)$ is $K_{4}$, we can assume wlog that the vertices of $C$ are $a, b, d, f$ in order on $C$, and that $a$ attaches to $A \in G-V(C), b$ to $B \in G-V(C)$, and $d$ to $D \in G-V(C)$ with $A, B, D$ distinct. Now $f$ must attach also to a vertex $F \in G-V(C)$. If $F \neq B$, then $G-E(C)$ is 2-connected. Suppose $F=B$ and let $C^{\prime}$ be the cycle with edges $a d, d f, f b, b a$. Then $G-E\left(C^{\prime}\right)$ is 2-connected.

Lemma 3.6. Let $N>5$ be an integer and assume Theorem 1.2 holds for all graphs $G^{\prime}$ with $\left|V\left(G^{\prime}\right)\right|=N-2$. Let $G$ be a 3-connected graph of minimum degree 4 with $|V(G)|=N$. Let $C$ be the even cycle of Lemma 3.2 and suppose $C$ has order 4 with one chord. If $G-V(C)$ is 2-connected, then there is an even cycle $C^{\prime}$ in $G$ such that $G-V\left(C^{\prime}\right)$ is non-empty and connected, and $G-E\left(C^{\prime}\right)$ is 2-connected. Further, one cannot form a triangle in $G$ entirely from chords of $C^{\prime}$.

Proof. As in Lemma 3.5, let the vertices of $C$ be $a, b, d, f$, in order on $C$, with the edge $a d$ being the unique chord of $C$. Consider first the case when $G-V(C)=K_{2}$. Then both vertices of the $K_{2}$ attach to $b$ and $f$. One vertex of the $K_{2}$ attaches to $a$ and the other to $d$. We conclude $G-E(C)$ is 2-connected. Next consider the case when $G-V(C)$ is 2-connected with at least three vertices. Suppose $a$ attaches to $A \in G-V(C)$ and $d$ to $D \in G-V(C)$. If $D \neq A$ then it is clear $G-E(C)$ is 2-connected so we shall assume $D=A$.

We consider the possible vertices of attachment of $b$ and $f$ in $G-V(C)$. Since $G$ is 3-connected, $b$ attaches to $B \in G-V(C)$ and $f$ to $F \in G-V(C)$ with 
$A, B, F$ distinct. Suppose now one of $b$ or $f$, say $b$, also attach to $A$. Then we may form a cycle $C^{\prime}$ of order 4 consisting of the edges $A a, a d, d b, b A$. Now $G-V\left(C^{\prime}\right)$ is connected and $G-E\left(C^{\prime}\right)$ is 2-connected. Hence we can assume that neither $b$ nor $f$ attaches to $A$.

Let us next suppose $G-V(C)=K_{3}$, whence the vertices are $A, B, F$ as above, with $A$ attaching to $a$ and $d$ in $C$ while $B$ and $F$ both attach to $b$ and $f$ in $C$. Let $C^{\prime}$ be the cycle of order 4 consisting of the edges $A a, a b, b B, B A$. Then $G-V\left(C^{\prime}\right)$ is connected and $G-E\left(C^{\prime}\right)$ is 2-connected.

Then, we can assume that $G-V(C)$ has at least four vertices and that there are distinct vertices $A, B, F$ of $G-V(C)$ with the properties:

(1) $a$ and $d$ attach to $A, b$ attaches to $B$, and $f$ to $F$;

(2) $A$ is the only vertex of attachment of $a$ and $d$ in $G-V(C)$;

(3) $b$ and $f$ attach to at least two vertices of $G-V(C)$ but do not attach to $A$.

Let $G^{\prime \prime}$ be the graph obtained from $G$ by contracting $f$ to $a$ and $d$ to $b$. One sees that $G^{\prime \prime} \neq K_{5}$ is 3-connected of minimum degree 4, and $\left|V\left(G^{\prime \prime}\right)\right|=N-2$. Hence by our assumption in the statement of the Lemma, there is an even cycle $C^{\prime \prime}$ in $G^{\prime \prime}$ with the property that $G^{\prime \prime}-V\left(C^{\prime \prime}\right)$ is non-empty and connected, and $G^{\prime \prime}-E\left(C^{\prime \prime}\right)$ is 2-connected. Further, one cannot form a triangle in $G^{\prime \prime}$ entirely from chords of $C^{\prime \prime}$. Let $a^{\prime \prime}$ be the vertex of $G^{\prime \prime}$ corresponding to the contraction of the edge $a f$ in $G$, and similarly let $b^{\prime \prime}$ be the vertex of $G^{\prime \prime}$ corresponding to the contraction of $b d$. If $V\left(C^{\prime \prime}\right)$ does not contain $a^{\prime \prime}$ or $b^{\prime \prime}$, then let $C^{\prime}$ be the cycle in $G$ identical to $C^{\prime \prime}$ in $G^{\prime \prime}$. It is clear that $G-V\left(C^{\prime}\right)$ is non-empty and connected, and $G-E\left(C^{\prime}\right)$ is 2-connected.Since there is a one-one correspondence between chords of $C^{\prime}$ and chords of $C^{\prime \prime}$, it follows that one cannot form a triangle in $G$ entirely from chords of $C^{\prime}$.

Next suppose $V\left(C^{\prime \prime}\right)$ contains $a^{\prime \prime}$ but not $b^{\prime \prime}$. Consider the two vertices of $C^{\prime \prime}$, which are adjacent to $a^{\prime \prime}$. If neither of these vertices is $A$ then we define the cycle $C^{\prime}$ in $G$ as the cycle identical to $C^{\prime \prime}$ in $G^{\prime \prime}$ but with the vertex $a^{\prime \prime} \in G^{\prime \prime}$ replaced by $f \in G$. Then $G-V\left(C^{\prime}\right)$ is non-empty and connected, and $G-E\left(C^{\prime}\right)$ is 2-connected. Observe that every chord of $C^{\prime}$ corresponds to a chord of $C^{\prime \prime}$. If $A \notin V\left(C^{\prime \prime}\right)$ then there is a one-one correspondence between chords of $C^{\prime}$ and chords of $C^{\prime \prime}$. If $A \in V\left(C^{\prime \prime}\right)$ the chord $a^{\prime \prime} A$ belongs to $C^{\prime \prime}$ but not to $C^{\prime}$. We conclude a triangle cannot be formed from the chords of $C^{\prime}$.

Suppose now $A$ is adjacent to $a^{\prime \prime}$ on $C^{\prime \prime}$ and $b^{\prime \prime} \notin V\left(C^{\prime \prime}\right)$. We can assume wlog that the other vertex adjacent to $a^{\prime \prime}$ on $C^{\prime \prime}$ is $F$. We define the cycle $C^{\prime}$ in $G$ as identical to $C^{\prime \prime}$ outside the $\operatorname{arc} F a^{\prime \prime} A$, but with the $\operatorname{arc} F a^{\prime \prime} A$ replaced by the arc $F f d a A$ in $G$. Note that $C^{\prime}$ has two more vertices than $C^{\prime \prime}$ and hence is even. We see again that $G-V\left(C^{\prime}\right)$ is non-empty and connected, and $G-E\left(C^{\prime}\right)$ is 2-connected. Observe that $C^{\prime}$ contains the chords $f a$ and $d A$, neither of which correspond to a chord of $C^{\prime \prime}$. Now $f a$ is the only chord of $C^{\prime}$ with vertex $a$, and $d A$ is the only chord of $C^{\prime}$ with vertex $d$. Hence a triangle consisting of chords of $C^{\prime}$ does not 
contain either of the chords $f a$ or $d A$. It follows that a triangle cannot be formed from chords of $C^{\prime}$.

Suppose now $V\left(C^{\prime \prime}\right)$ contains both $a^{\prime \prime}$ and $b^{\prime \prime}$ and $a^{\prime \prime}, b^{\prime \prime}$ are adjacent as vertices of $C^{\prime \prime}$. Assume $A$ is the other adjacent vertex of either $a^{\prime \prime}$ or $b^{\prime \prime}$, say $a^{\prime \prime}$. We define the cycle $C^{\prime}$ in $G$ as the cycle identical to $C^{\prime \prime}$ in $G^{\prime \prime}$, but with the arc $A a^{\prime \prime} b^{\prime \prime}$ replaced by the arc $A a f d b$. It is easy to see that both $G-V\left(C^{\prime}\right)$ and $G-E\left(C^{\prime}\right)$ are connected. The graph $G-E\left(C^{\prime}\right)$ is also 2-connected. To see that $G-E\left(C^{\prime}\right)-\{a\}$ is connected, note that we use the fact that $G^{\prime \prime}-E\left(C^{\prime \prime}\right)-\left\{b^{\prime \prime}\right\}$ is connected. Observe that $C^{\prime}$ contains the chords $d A, a b$, and $a d$. Arguing as in the previous paragraph, we see that a triangle in $G$, consisting of chords of $C^{\prime}$, does not contain any of the chords $d A, a b$, or $a d$. Hence a triangle cannot be formed from the chords of $C^{\prime}$.

Suppose next that $a^{\prime \prime}, b^{\prime \prime}$ are adjacent vertices of $C^{\prime \prime}$ but neither is adjacent to $A$. We may assume therefore that $F$ is the other vertex adjacent to $a^{\prime \prime}$ on $C^{\prime \prime}$ and $B$ the other vertex adjacent to $b^{\prime \prime}$. To obtain $C^{\prime}$ from $C^{\prime \prime}$, we replace the $\operatorname{arc} F a^{\prime \prime} b^{\prime \prime} B$ on $C^{\prime \prime}$ by the arc $F f d a b B$ on $C^{\prime}$. It is easy to see that $G-V\left(C^{\prime}\right)$ is non-empty and connected while $G-E\left(C^{\prime}\right)$ is 2-connected. Now $C^{\prime}$ contains the chords $f a$ and $d b$, which do not correspond to chords of $C^{\prime \prime}$. Again we see that a triangle in $G$ consisting of chords of $C^{\prime}$ does not contain either of the chords $f a$ or $d b$. We conclude a triangle cannot be formed from chords of $C^{\prime}$.

Finally, we suppose $V\left(C^{\prime \prime}\right)$ contains both $a^{\prime \prime}$ and $b^{\prime \prime}$ but $a^{\prime \prime}, b^{\prime \prime}$ are not adjacent as vertices of $C^{\prime \prime}$. Suppose $A \notin V\left(C^{\prime \prime}\right)$. We obtain $C^{\prime}$ from $C^{\prime \prime}$ by replacing $a^{\prime \prime}$ by $f$ and $b^{\prime \prime}$ by $b$. Since $A \notin V\left(C^{\prime \prime}\right)$ it follows that $G-V\left(C^{\prime}\right)$ is non-empty and connected. It is easy to see that $G-E\left(C^{\prime}\right)$ is 2-connected. Since there is a oneone correspondence between chords of $C^{\prime}$ and chords of $C^{\prime \prime}$, apart from the chord $a^{\prime \prime} b^{\prime \prime}$, it is not possible to form a triangle from chords of $C^{\prime}$.

Suppose $A \in V\left(C^{\prime \prime}\right)$. Then $A$ must be adjacent on $C^{\prime \prime}$ to either $a^{\prime \prime}$ or $b^{\prime \prime}$. Otherwise the triangle with edges $A a^{\prime \prime}, a^{\prime \prime} b^{\prime \prime}, b^{\prime \prime} A$ consists of chords of $C^{\prime \prime}$. Suppose $A$ is adjacent on $C^{\prime \prime}$ to both $a^{\prime \prime}$ and $b^{\prime \prime}$. Then we obtain $C^{\prime}$ from $C^{\prime \prime}$ by replacing the arc $a^{\prime \prime} A b^{\prime \prime}$ by the arc $f a A d b$. It is clear that $G-V\left(C^{\prime}\right)$ is non-empty and connected while $G-E\left(C^{\prime}\right)$ is 2-connected. The chords of $C^{\prime}$, excepting the chords $a d$ and $f d$, are in one-one correspondence to the chords of $C^{\prime \prime}$. Hence it is not possible to form a triangle from chords of $C^{\prime}$.

The last case we need to consider is where $a^{\prime \prime}, b^{\prime \prime}, A \in V\left(C^{\prime \prime}\right)$ and $A$ is adjacent on $C^{\prime \prime}$ to $a^{\prime \prime}$ but not to $b^{\prime \prime}$. Then we obtain $C^{\prime}$ from $C^{\prime \prime}$ by replacing $b^{\prime \prime}$ by $b$ and, in the case of $a^{\prime \prime}$, acting as when $V\left(C^{\prime \prime}\right)$ was assumed to contain $a^{\prime \prime}$ and not $b^{\prime \prime}$. Thus, we replace the arc $F a^{\prime \prime} A$ in $C^{\prime \prime}$ by the arc $F f d a A$ in $C^{\prime}$. We conclude as before that $G-V\left(C^{\prime}\right)$ is non-empty and connected, and $G-E\left(C^{\prime}\right)$ is 2-connected. It is also clear that one cannot form a triangle in $G$ from chords of $C^{\prime}$.

Proof of Proposition 3.1. If $C$ has order larger than 4, then the result follows from Lemmas 3.3, 3.4 and Corollary 3.1. If $C$ has order 4 then $G-V(C)$ is not a vertex graph since $|V(G)| \geq 6$. In that case, the result follows from Lemmas 3.5, and 3.6. 


\section{4. $G-V(C)$ SEPARABLE IMPLIES $C$ OF ORDER 4 OR 6}

Our goal in this section is the prove the following.

Proposition 4.1. Suppose $G$ is a 3-connected graph of minimum degree 4 and $C$ is an even cycle satisfying (a) and (b) of Lemma 3.2. If $G-V(C)$ is separable then $|V(C)|=4$ or 6 .

To prove Proposition 4.1 we consider the block decomposition of $G-V(C)$. Now $G-V(C)$ has at least two endblocks, one of which may be the largest block $B^{*}$ of Lemma 3.2. We consider an endblock other than $B^{*}$. By Lemma 3.1, this block is either a $K_{2}$ or an odd cycle. Let $a_{1}$ be a vertex of this block, which is not a cutvertex of $G-V(C)$. Since $a_{1}$ has degree 4 in $G, a_{1}$ must have at least two vertices of attachment $a_{2}, a_{3}$ in $C$.

Lemma 4.1. Suppose the arc joining $a_{2}$ to $a_{3}$ in $C$ is odd. Then $|V(C)|=4$.

Proof. Observe that there are two vertices of $C$, which attach to vertices of $G-V(C)$ other than the vertex $a_{1}$. This follows from the 3-connectedness of $G$. Denote these vertices by $d, f$. Suppose $d$ does not coincide with either $a_{2}$ or $a_{3}$ and let $\operatorname{arc}\left(a_{2}, a_{3}\right)$ be the arc of $C$ joining $a_{2}$ to $a_{3}$, which does not contain $d$. Let $C^{\prime}$ be the cycle consisting of $a_{1}$ and $\operatorname{arc}\left(a_{2}, a_{3}\right)$. Since $C^{\prime}$ is even, it follows from (b) of Lemma 3.2 that $V(C)-V\left(\operatorname{arc}\left(a_{2}, a_{3}\right)\right)=\{d\}$. We conclude that if neither $d$ nor $f$ coincides with $a_{2}$ or $a_{3}$ then $|V(C)|=4$.

Assume now that $d=a_{2}, f \neq a_{3}$, and $|V(C)|>4$. We have then from the above argument that if $\operatorname{arc}\left(a_{2}, a_{3}\right)$ is the $\operatorname{arc}$ of $C$ joining $a_{2}$ to $a_{3}$, which does not contain $f$ then $V(C)-V\left(\operatorname{arc}\left(a_{2}, a_{3}\right)\right)=\{f\}$. Suppose first that no internal vertex of $\operatorname{arc}\left(a_{2}\right.$, $\left.a_{3}\right)$ attaches to a vertex of $G-V(C)$. Since $|V(C)|>4$, the $\operatorname{arc}, \operatorname{arc}\left(a_{2}, a_{3}\right)$, has at least five vertices. Consider the graph $H$ on the vertices of $\operatorname{arc}\left(a_{2}, a_{3}\right)$. Then $H$ is connected, $|V(H)| \geq 5$, and all but two vertices of $H$ have degree at least 3 (since they could attach to $f$ ). It follows from Lemma 2.1 that $H$ contains an even cycle. Since $f$ attaches to $G-V(C)$ we obtain a contradiction to (b) of Lemma 3.2. Suppose next that an internal vertex of $\operatorname{arc}\left(a_{2}, a_{3}\right)$ does attach to a vertex of $G-V(C)$. From previous argument, we can assume this vertex must be $a_{1}$. Moreover, we can similarly argue that there is precisely one internal vertex of $\operatorname{arc}\left(a_{2}, a_{3}\right)$, which attaches to $a_{1}$, say the vertex $a_{4}$, and the $\operatorname{arc}, \operatorname{arc}\left(a_{2}, a_{4}\right) \subset$ $\operatorname{arc}\left(a_{2}, a_{3}\right)$ is even. Let $H$ be the graph on the vertices of $\operatorname{arc}\left(a_{2}, a_{3}\right)$. Then $H$ is connected with $|V(H)| \geq 5$. The vertices $a_{2}, a_{3}$ have degree at least 1 in $H$ and $a_{4}$ has degree at least 2 . All other vertices have degree at least 3. Observe also that $H$ contains two paths, from $a_{4}$ to $a_{2}$ and from $a_{4}$ to $a_{3}$, which contain an even number of vertices and are disjoint except for the common vertex $a_{4}$. It follows by Lemma 2.1 that $H$ contains an even cycle. Note that the evenness of the paths $a_{4}$ to $a_{2}$ and $a_{4}$ to $a_{3}$ is required to rule out the configuration in Figure 2. Again, we have a contradiction to (b) of Lemma 3.2 since $f$ attaches to $G-V(C)$.

We can assume now that $d=a_{2}, f=a_{3},|V(C)|>4$. Since $G$ is 3-connected, there is a third vertex $a_{4}$ of $C$, which attaches to $a_{1}$. Let $\operatorname{arc}\left(a_{2}, a_{3}\right)$ be the $\operatorname{arc}$ of $C$ 


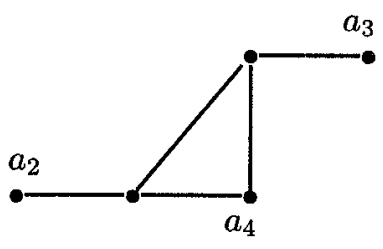

FIGURE 2.

joining $a_{2}$ to $a_{3}$, which contains $a_{4}$. Thus we must have that $\operatorname{arc}\left(a_{2}, a_{4}\right) \subset$ $\operatorname{arc}\left(a_{2}, a_{3}\right)$ is even, and that $a_{4}$ is the only internal vertex of $\operatorname{arc}\left(a_{2}, a_{3}\right)$, which attaches to $G-V(C)$. Suppose now there is a vertex $a_{5}$ of $V(C)-V\left(\operatorname{arc}\left(a_{2}, a_{3}\right)\right)$, which attaches to $a_{1}$. Then the arc joining $a_{2}$ to $a_{5}$ must be even, in order not to contradict (b) of Lemma 3.2. In that case, the arc joining $a_{4}$ to $a_{5}$ is odd. Since $d, f \neq a_{4}, a_{5}$ we conclude $C$ has order 4. Suppose finally that no vertex of $V(C)-V\left(\operatorname{arc}\left(a_{2}, a_{3}\right)\right)$ attaches to $G-V(C)$. Let $H$ be the graph on the vertices $V(C)-\left\{a_{2}\right\}$. Then $H$ is connected, $|V(H)| \geq 5$, and all but two vertices of $H$ have degree at least 3. It follows from Lemma 2.1 that $H$ contains an even cycle. Since $a_{2}$ attaches to $G-V(C)$, we have a contradiction to (b) of Lemma 3.2.

Corollary 4.1. Suppose $G-V(C)$ is separable and has an endblock which is a $K_{2}$. Then $C$ has order 4.

Proof. The endblock, which is a $K_{2}$, has a vertex $a_{1}$ of degree 1 in $G-V(C)$. Hence $a_{1}$ has three vertices of attachment in $C$. Of these, we can choose two of them, $a_{2}, a_{3}$ such that the arc of $C$ joining $a_{2}$ to $a_{3}$ is odd.

Lemma 4.2. Suppose $G-V(C)$ is separable and has at least two endblocks different from the largest block $B^{*}$ of Lemma 3.2. Then $C$ has order 4.

Proof. By Corollary 4.1 we may assume that two of the endblocks are odd cycles. We consider one of these odd cycles, and let $a_{1}, a_{1}^{\prime}$ be adjacent vertices of the cycle, neither of which is a cutvertex of $G-V(C)$. It follows that both $a_{1}, a_{1}^{\prime}$ have degree 2 in $G-V(C)$, whence they have each two vertices of attachment in $C$. By Lemma 4.1 we can assume there are vertices $a_{2}, a_{3}$ of $C$ such that the arc of $C$ joining $a_{2}$ to $a_{3}$ is even and $a_{1}$ attaches to $a_{2}$ while $a_{1}^{\prime}$ attaches to $a_{3}$. It is clear that the cycle consisting of $\operatorname{arc}\left(a_{2}, a_{3}\right)$ and the edge $a_{1} a_{1}^{\prime}$ is even. We can make similar assumptions about the second endblock, which is an odd cycle. Hence this cycle has adjacent vertices $b_{1}, b_{1}^{\prime}$, which are not cutvertices of $G-V(C)$. The vertex $b_{1}$ attaches to the vertex $b_{2}$ of $C$, while $b_{1}^{\prime}$ attaches to $b_{3}$ in $C$ where the arc of $C$ joining $b_{2}$ to $b_{3}$ is even.

We consider the situation where neither $b_{2}$ nor $b_{3}$ coincides with $a_{2}$ or $a_{3}$. Suppose first that $b_{2}$ and $b_{3}$ are on the same arc of $C$ joining $a_{2}$ to $a_{3}$. Let 
$\operatorname{arc}\left(b_{2}, b_{3}\right)$ be the arc joining $b_{2}$ to $b_{3}$, which does not include $a_{2}, a_{3}$. We can form the even cycle $C^{\prime}$ consisting of $b_{1}, b_{1}^{\prime}$ and $\operatorname{arc}\left(b_{2}, b_{3}\right)$, which yields a contradiction to (b) of Lemma 3.2 unless $V(C)-V\left(\operatorname{arc}\left(b_{2}, b_{3}\right)\right)=\left\{a_{2}, a_{3}\right\}$. Arguing similarly for $a_{1}, a_{1}^{\prime}, a_{2}, a_{3}$, we conclude $C$ has order 4 . Next suppose $b_{2}$ and $b_{3}$ are on different arcs of $C$ joining $a_{2}$ to $a_{3}$. Arguing as above, we conclude that $C$ has order 6. We may also assume that $b_{2}$ is adjacent to $a_{2}$ on $C$ and $b_{3}$ adjacent to $a_{3}$. Consider now the largest block $B^{*}$ of Lemma 3.2 and assume $B^{*}$ has at most two cutvertices in $G-V(C)$. Since $G$ is 3-connected there is a vertex $v$ of $C$, which attaches to a vertex of $B^{*}$ that is not a cutvertex of $G-V(C)$. Suppose now $v \neq a_{2}, a_{3}$ and is on the same arc of $C$ joining $a_{2}$ to $a_{3}$, which contains $b_{2}$. Let $C^{\prime}$ be the even cycle consisting of $a_{1}, a_{1}^{\prime}$ and the $\operatorname{arc}, \operatorname{arc}\left(a_{2}, a_{3}\right)$, which does not contain $b_{2}$. Let $b \in V\left(B^{*}\right)$ be the cutvertex of $G-V(C)$ whose removal disconnects $B^{*}$ from the vertex $b_{1}$. Then there is a path $P\left(b, b_{1}\right)$ in $G-V(C)$ from $b$ to $b_{1}$, which intersects $B^{*}$ only in the vertex $b$ and does not contain the vertices $a_{1}, a_{1}^{\prime}$. Let $B^{* *}$ be the graph on the vertices $V\left(B^{*}\right) \cup V\left(P\left(b, b_{1}\right)\right) \cup\left\{v, b_{2}\right\}$. Then $B^{* *}$ is 2-connected and is disjoint from the even cycle $C^{\prime}$. This contradicts (a) of Lemma 3.2. Let us assume now that $B^{*}$ has at least three cutvertices in $G-V(C)$. Let $b$ be as before and $a \in V\left(B^{*}\right)$ be the cutvertex of $G-V(C)$, whose removal disconnects $B^{*}$ from the vertex $a_{1}$. Then there is an endblock $B$ of $G-V(C)$, which remains connected to $B^{*}$ if either $a$ or $b$ is removed from $G-V(C)$. Since there is a vertex $v$ of $C$, which attaches to $B$ we can argue as above to obtain a contradiction to (a) of Lemma 3.2.

We consider next the situation where $b_{2}=a_{2}$ but $b_{3} \neq a_{3}$. Let $\operatorname{arc}\left(a_{2}, a_{3}\right)$ be the arc of $C$ joining $a_{2}$ to $a_{3}$, which does not contain $b_{3}$. We can form the even cycle $C^{\prime}$ consisting of $a_{1}, a_{1}^{\prime}$, and $\operatorname{arc}\left(a_{2}, a_{3}\right)$. Hence by Lemma 3.2(b), we must have $V(C)-V\left(\operatorname{arc}\left(a_{2}, a_{3}\right)\right)=\left\{b_{3}, v\right\}$, and $b_{3}$ is adjacent to $b_{2}$ (since the arc joining $b_{2}$ to $b_{3}$ is even). Now by forming the cycle $b_{1}, b_{2}, b_{3}, b_{1}^{\prime}$ we see that Lemma 3.2(b) implies $C$ has order 4.

Finally we consider the situation where $b_{2}=a_{2}, b_{3}=a_{3}$. We first show that we may assume the only vertices of attachment of $a_{1}, a_{1}^{\prime}, b_{1}, b_{1}^{\prime}$ in $C$ are $b_{2}=a_{2}, b_{3}=a_{3}$. To see this note that both $b_{1}$ and $b_{1}^{\prime}$ have two vertices of attachment in $C$. Since $b_{1}$ attaches to $b_{2}$, let us assume the other vertex of attachment is $b_{2}^{\prime}$ in $C$. By Lemma 4.1 we can assume the arc of $C$ joining $b_{2}$ to $b_{2}^{\prime}$ is even. Suppose that $b_{2}^{\prime} \neq b_{3}$. Now $b_{1}^{\prime}$ has a second vertex of attachment in $C$ other than $b_{3}$. We denote it by $b_{3}^{\prime}$ and assume the arc of $C$ joining $b_{3}$ to $b_{3}^{\prime}$ is even. It follows that the arc of $C$ joining $b_{2}^{\prime}$ to $b_{3}^{\prime}$ is even. Since $b_{2}^{\prime} \neq a_{2}, a_{3}$ this puts us back in the cases already considered. We conclude that $C$ has order 4 .

Let us assume now that all of the vertices $a_{1}, a_{1}^{\prime}, b_{1}, b_{1}^{\prime}$ attach to both $a_{2}$ and $a_{3}$ and to no other vertices of $C$. Let $H$ be the graph on $V(C)-\left\{a_{2}, a_{3}\right\}$. Since $G$ is 3-connected it follows that $H$ is connected to $G-V(C)$ and $a_{1}, a_{1}^{\prime}$ are not vertices of attachment of $H$ in $G-V(C)$. Let $C^{\prime}$ be the cycle of order 4 consisting of the edges $a_{1} a_{2}, a_{2} a_{1}^{\prime}, a_{1}^{\prime} a_{3}, a_{3} a_{1}$. Since the graph on $V(G) \cup V(H)-$ $\left\{a_{1}, a_{1}^{\prime}, a_{2}, a_{3}\right\}$ is connected, we conclude from (b) of Lemma 3.2 that $C$ has order 4 . 
Lemma 4.3. Suppose $G-V(C)$ is separable and has two endblocks, one of the endblocks being the largest block $B^{*}$ of Lemma 3.2 and the other endblock an odd cycle. Then $C$ has order 4 or 6.

Proof. Let $a_{1}, a_{1}^{\prime}$ be adjacent vertices of the endblock of $G-V(C)$, which is an odd cycle and assume neither $a_{1}$ nor $a_{1}^{\prime}$ is a cutvertex of $G-V(C)$. As in Lemma 4.2 we may assume that $a_{1}$ attaches to a vertex $a_{2}$ of $C$ and $a_{1}^{\prime}$ to a vertex $a_{3}$, such that the arc of $C$ joining $a_{2}$ to $a_{3}$ is even. Since $G$ is 3-connected, there are two vertices $d, f$ of $C$, which attach to two vertices of $B^{*}$ different from the cutvertex of $B^{*}$ in $G-V(C)$. Suppose first $d, f$ do not coincide with either $a_{2}$ or $a_{3}$. If $d$ and $f$ are on different arcs of $C$ joining $a_{2}$ to $a_{3}$ then it follows from (b) of Lemma 3.2 that $C$ has order 6 . If $d$ and $f$ are on the same arc of $C$ joining $a_{2}$ to $a_{3}$, let us denote by $\operatorname{arc}(d, f)$ the $\operatorname{arc}$ of $C$ joining $d$ to $f$, which does not include $a_{2}$ or $a_{3}$. Let $\operatorname{arc}\left(a_{2}, a_{3}\right)$ be the arc of $C$ joining $a_{2}$ to $a_{3}$, which does not include $d, f$. Then we may form the even cycle $C^{\prime}$ from $a_{1}, a_{1}^{\prime}$ and $\operatorname{arc}\left(a_{2}, a_{3}\right)$. Let $B^{* *}$ be the graph on $V\left(B^{*}\right) \cup V(\operatorname{arc}(d, f))$. Then $B^{* *}$ is 2-connected and lies outside the even cycle $C^{\prime}$. This contradicts (a) of Lemma 3.2.

We assume $d=a_{2}, f \neq a_{3}$. Let $\operatorname{arc}\left(a_{2}, a_{3}\right)$ be the arc of $C$ joining $a_{2}$ to $a_{3}$, which does not include $f$. It follows by (b) of Lemma 3.2 that $V(C)-$ $V\left(\operatorname{arc}\left(a_{2}, a_{3}\right)\right)=\{f, v\}$. Consider now an internal vertex $w$ of $\operatorname{arc}\left(a_{2}, a_{3}\right)$. If $w$ attaches to a vertex of $G-V(C)$ other than $a_{1}, a_{1}^{\prime}$ then by (b) of Lemma 3.2, we may conclude $C$ has order 6 . If $w$ attaches to $a_{1}$ then $\operatorname{arc}\left(a_{2}, w\right) \subset \operatorname{arc}\left(a_{2}, a_{3}\right)$ is even or else $C$ has order 4 . Let us assume now $C$ has order larger than 6 . By arguing as in Lemma 4.1, it follows that there is at most one internal vertex $a_{4}$ of $\operatorname{arc}\left(a_{2}, a_{3}\right)$, which attaches to a vertex of $G-V(C)$. Let $H$ be the graph on the vertices $V\left(\operatorname{arc}\left(a_{2}, a_{3}\right)\right) \cup\{v\}$. Thus $H$ is connected and has at least seven vertices. At most two vertices, $a_{2}$ or $a_{3}$, and $v$ have degree one in the graph, and all but the four vertices $a_{2}, a_{3}, a_{4}, v$ have degree at least three (since any vertex can attach to $f$ ). There is also a path in $H$, which goes through every vertex of $H$. This rules out the configuration in Figure 3. We conclude from Lemma 2.1 that $H$ must contain an even cycle. Since $f$ attaches to $G-V(C)$, this contradicts (b) of Lemma 3.2. We conclude $C$ has order 4 or 6 .

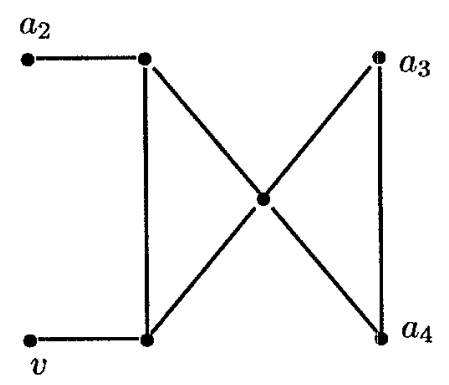

FIGURE 3. 
Finally we assume $d=a_{2}, f=a_{3}$. Suppose there is a vertex $f^{\prime} \in V(C)-$ $\left\{a_{2}, a_{3}\right\}$, which attaches to a vertex of $G-V(C)$ other than $a_{1}, a_{1}^{\prime}$. Then we may argue as in the previous paragraph to conclude $C$ has order 4 or 6 . Suppose next that there is a vertex $a_{4} \in V(C)-\left\{a_{2}, a_{3}\right\}$, which attaches to $a_{1}$. Then we may assume the arc of $C$ joining $a_{2}$ to $a_{4}$ is even. Consider the second vertex of attachment $a_{4}^{\prime}$ of $a_{1}^{\prime}$ in $C$. We may assume the arc of $C$ joining $a_{4}$ to $a_{4}^{\prime}$ is even. Since $f \neq a_{4}, a_{4}^{\prime}$, this puts us in the situation dealt with in the previous paragraph. We conclude that if $C$ has order larger than 6 , then $a_{2}, a_{3}$ are the vertices of attachment in $C$ of $a_{1}, a_{1}^{\prime}$. Further, no vertex of $V(C)-\left\{a_{2}, a_{3}\right\}$ attaches to $G-V(C)$. This contradicts the 3-connectedness of $G$.

Proof of Proposition 4.1. This follows from the previous lemmas. Note that if $C$ has order 6 then $G-V(C)$ has just two endblocks, one of which is $B^{*}$.

\section{5. $G-V(C)$ SEPARABLE, $C$ OF ORDER 6}

In this section we prove the following.

Proposition 5.1. Suppose $G$ is a 3-connected graph of minimum degree 4 and $C$ is a cycle of order 6 satisfying (a) and (b) of Lemma 3.2. If $G-V(C)$ is separable then $G-E(C)$ is 2-connected.

To prove Proposition 5.1 we first observe from Lemmas 4.2 and 4.3 that $G-V(C)$ has exactly two endblocks, one of which is the largest block $B^{*}$ of Lemma 3.2. By Corollary 4.1 the other endblock is an odd cycle. Let $a_{1}, a_{1}^{\prime}$ be adjacent vertices of the cycle, neither of which is a cutvertex of $G-V(C)$. Then both $a_{1}$ and $a_{1}^{\prime}$ have two vertices of attachment in $C$.

Lemma 5.1. Suppose $a_{1}$ attaches to a vertex $a_{2} \in V(C)$ and $a_{1}^{\prime}$ to $a_{3} \in V(C)$. Then $a_{2}$ and $a_{3}$ are not adjacent on $C$.

Proof. Let us assume $a_{2}$ and $a_{3}$ are adjacent on $C$. Arguing as in Lemma 4.3 we see that there are two vertices $d, f$ of $C$, which attach to two vertices of $B^{*}$ different from the cutvertex of $B^{*}$ in $G-V(C)$. If any vertex in $V(C)-\left\{a_{2}, a_{3}\right\}$ attaches to a vertex of $G-V(C)$ other than $a_{1}, a_{1}^{\prime}$ then we have a contradiction to (b) of Lemma 3.2, since we can form the cycle of order 4 with edges $a_{1} a_{2}, a_{2} a_{3}, a_{3} a_{1}^{\prime}, a_{1}^{\prime} a_{1}$. We conclude that $d=a_{2}, f=a_{3}$. Since $G$ is 3 -connected we may also conclude that there is at least one vertex of $V(C)-\left\{a_{2}, a_{3}\right\}$ which attaches to $a_{1}$ or $a_{1}^{\prime}$. Suppose the vertex is $a_{2}^{\prime}$ and that it attaches to $a_{1}$. In view of Lemma 4.1 the arc of $C$ joining $a_{2}$ to $a_{2}^{\prime}$ is even. Since the arc of $C$ joining $a_{3}$ to $a_{2}^{\prime}$ is odd, it follows again from Lemma 4.1 that the second vertex of attachment of $a_{1}^{\prime}$ in $C$ cannot be $a_{2}^{\prime}$. Suppose it is $a_{3}^{\prime}$ and let us assume $a_{3}^{\prime} \neq a_{2}$. Let $\operatorname{arc}\left(a_{2}^{\prime}, a_{3}^{\prime}\right)$ be the arc of $C$, which does not contain $a_{2}, a_{3}$. Then $\operatorname{arc}\left(a_{2}^{\prime}, a_{3}^{\prime}\right)$ is even, whence one can form an even cycle $C^{\prime}$ consisting of $a_{1}, a_{1}^{\prime}$ and $\operatorname{arc}\left(a_{2}^{\prime}, a_{3}^{\prime}\right)$. Since $C^{\prime}$ is disjoint from $d, f$, we may argue as in Lemma 4.3 to obtain a contradiction to (a) of Lemma 3.2. 
Assume now that $a_{3}^{\prime}=a_{2}$. If $a_{2}^{\prime}$ is adjacent to $a_{2}$, then by forming the cycle of order 4 consisting of the edges $a_{1} a_{2}^{\prime}, a_{2}^{\prime} a_{2}, a_{2} a_{1}^{\prime}, a_{1}^{\prime} a_{1}$, we obtain a contradiction to (b) of Lemma 3.2. We conclude that the only vertices of attachment of $a_{1}, a_{1}^{\prime}$ in $C$ are $a_{2}, a_{3}, a_{2}^{\prime}$. The vertex $a_{1}$ attaches to $a_{2}, a_{2}^{\prime}$ and the arc joining $a_{2}$ to $a_{2}^{\prime}$ has order 4. The vertex $a_{1}^{\prime}$ attaches to $a_{2}$ and $a_{3}$ with $a_{2}, a_{3}$ adjacent. We can denote the vertices of $C$ in order as $a_{2}, a_{3}, a_{4}, a_{2}^{\prime}, a_{5}, a_{6}$. We have already seen that $a_{4}, a_{5}, a_{6}$ have no vertices of attachment in $G-V(C)$. Suppose now the edge $a_{2} a_{4}$ exists. Let $C^{\prime}$ be the cycle of order 4 consisting of the edges $a_{1}^{\prime} a_{2}, a_{2} a_{4}, a_{4} a_{3}, a_{3} a_{1}^{\prime}$. Since $a_{2}^{\prime}$ attaches to $a_{1}$ it is clear that we have a contradiction to (b) of Lemma 3.2. Since the vertex $a_{4}$ has minimum degree 4 in $G$ we conclude that the edge $a_{4} a_{6}$ must exist. Hence we can form the cycle $C^{\prime}$ of order 4 consisting of the edges $a_{6} a_{5}, a_{5} a_{2}^{\prime}, a_{2}^{\prime} a_{4}, a_{4} a_{6}$. Since $C^{\prime}$ is disjoint from $d, f$ we can argue again as in Lemma 4.3 to obtain a contradiction to (a) of Lemma 3.2.

Lemma 5.2. The vertices $a_{1}, a_{1}^{\prime}$ have precisely 4 vertices of attachment in $C$.

Proof. Note that by Lemma 4.1 the vertices $a_{1}, a_{1}^{\prime}$ have at most four vertices of attachment in $C$. By Lemma 5.1 we can assume that $a_{1}$ attaches to a vertex $a_{2}$ of $C, a_{1}^{\prime}$ to a vertex $a_{3}$ of $C$ and the arc joining $a_{2}$ to $a_{3}$ has order 4. Suppose now $a_{1}$ also attaches to a vertex $a_{2}^{\prime} \neq a_{3}$ of $C$. Then by Lemma 4.1 the vertices $a_{2}, a_{2}^{\prime}$ are adjacent on $C$. If $a_{1}^{\prime}$ attaches to $a_{2}$ then we have a contradiction to Lemma 5.1. If $a_{1}^{\prime}$ attaches to $a_{2}^{\prime}$ then we have a contradiction to Lemma 4.1. We conclude that $a_{1}, a_{1}^{\prime}$ have either four vertices of attachment in $C$ or two vertices of attachment.

Assume now $a_{1}, a_{1}^{\prime}$ have two vertices of attachment in $C$, whence $a_{1}, a_{1}^{\prime}$ attach to both $a_{2}, a_{3}$. Let $C^{\prime}$ be the cycle of order 4 consisting of the edges $a_{1} a_{2}, a_{2} a_{1}^{\prime}$, $a_{1}^{\prime} a_{3}, a_{3} a_{1}$. Observe now that since $G$ is 3 -connected the graph on $V(G)-V\left(C^{\prime}\right)$ is connected. Hence we have a contradiction to (b) of Lemma 3.2.

From Lemmas 5.1 and 5.2, we see that $a_{1}$ attaches to two vertices $a_{2}, a_{2}^{\prime}$ of $C$ and $a_{1}^{\prime}$ to two different vertices $a_{3}, a_{3}^{\prime}$. The arc joining $a_{2}$ to $a_{3}$ has order $4, a_{2}^{\prime}$ is adjacent on $C$ to $a_{2}, a_{3}^{\prime}$ is adjacent on $C$ to $a_{3}$, and the arc joining $a_{2}^{\prime}$ to $a_{3}^{\prime}$ has order 4. We have also seen that there are vertices $d, f$ of $C$, which attach to two vertices of $B^{*}$ different from the cutvertex of $B^{*}$ in $G-V(C)$. We then have the following.

Lemma 5.3. Suppose the arc of $C$ joining $d$ to $f$ has order 4. Then $G-E(C)$ is 2-connected.

Proof. Let us assume that $d$ is not a vertex of attachment in $C$ of $a_{1}, a_{1}^{\prime}$. Then wlog, we may write the vertices of $C$ in order as $a_{2}, d, a_{3}^{\prime}, a_{3}, f, a_{2}^{\prime}$. Suppose now $d$ has a second vertex of attachment in $G-V(C)$. This vertex of attachment cannot be $a_{1}$ or $a_{1}^{\prime}$. Let $C^{\prime}$ be the cycle consisting of the edges $a_{1} a_{2}, a_{2} a_{2}^{\prime}, a_{2}^{\prime} f, f a_{3}$, $a_{3} a_{1}^{\prime}, a_{1}^{\prime} a_{1}$. By arguing as in Lemma 4.3 we see we can construct a block containing $B^{*}$ and $d$, which is disjoint from $C^{\prime}$. This contradicts (a) of Lemma 3.2. Suppose next that the edge $d a_{3}$ exists. Let $C^{\prime}$ be the cycle consisting of the edges $a_{1}^{\prime} a_{3}, a_{3} d, d a_{3}^{\prime}, a_{3}^{\prime} a_{1}^{\prime}$. Since $f$ attaches to a vertex of $G-V(C)$ other than $a_{1}^{\prime}$ we have a contradiction to (b) of Lemma 3.2. Suppose the edge $d a_{2}^{\prime}$ exists. Let $C^{\prime}$ be 
the cycle consisting of the edges $a_{1} a_{2}, a_{2} d, d a_{2}^{\prime}, a_{2}^{\prime} a_{1}$. Since $f$ attaches to a vertex of $G-V(C)$ other than $a_{1}$ we have again a contradiction to (b) of Lemma 3.2. Since $d$ has minimum degree 4 in $G$, we conclude that the edge $d f$ must exist.

Consider next the vertex $a_{2}$. Suppose the edge $a_{2} f$ exists. Let $C^{\prime}$ be the cycle consisting of the edges $a_{1} a_{2}, a_{2} f, f a_{2}^{\prime}, a_{2}^{\prime} a_{1}$. Since $d$ attaches to a vertex of $G-V(C)$ other than $a_{1}$, we have a contradiction to (b) of Lemma 3.2. Next suppose the edge $a_{2} a_{3}$ exists. Let $C^{\prime}$ be the cycle consisting of the edges $a_{1} a_{2}, a_{2} a_{3}, a_{3} a_{1}^{\prime}, a_{1}^{\prime} a_{1}$. Since $d$ and $f$ attach to vertices of $G-V(C)$ other than $a_{1}, a_{1}^{\prime}$, we obtain a contradiction to (b) of Lemma 3.2. A similar argument rules out the possibility of an edge $a_{2} a_{3}^{\prime}$. We conclude that, since $a_{2}$ has minimum degree 4 , it must attach to a vertex of $G-V(C)$ other than $a_{1}, a_{1}^{\prime}$. If this vertex of attachment is different from the vertex of attachment of $d$ in $B^{*}$ then we can construct a block containing $B^{*}$ and the vertices $a_{2}, d$ of $C$. This block is disjoint from the cycle $C^{\prime}$ consisting of the edges $a_{1} a_{2}^{\prime}, a_{2}^{\prime} f, f a_{3}, a_{3} a_{3}^{\prime}, a_{3}^{\prime} a_{1}^{\prime}, a_{1}^{\prime} a_{1}$. We have therefore a contradiction to (a) of Lemma 3.2. It follows that $a_{2}$ and $d$ have the same vertex of attachment in $G-V(C)$ and it is a non-cutvertex of $B^{*}$. A similar argument shows that $a_{3}^{\prime}$ has a vertex of attachment in $B^{*}$, which is the same as the vertex of attachment of $d$. By symmetry, we can make similar conclusions about the vertices $a_{2}^{\prime}, f, a_{3}$.

Next we wish to show that $G-V(C)$ has just two blocks. One of the blocks is the largest block $B^{*}$ and the other is an odd cycle, which we shall show must be a triangle. Suppose first that the odd cycle containing $a_{1}, a_{1}^{\prime}$ is not a triangle. Then we may assume wlog that there is a vertex $a_{1}^{\prime \prime}$ of the cycle adjacent to $a_{1}^{\prime}$, which is not a cutvertex of $G-V(C)$. Hence $a_{1}^{\prime \prime}$ has two vertices of attachment in $C$. It is easy to see that no matter which vertex of attachment $a_{1}^{\prime \prime}$ has in $C$ one can form a cycle of order 4 with either $a_{1}, a_{1}^{\prime}, a_{1}^{\prime \prime}$ and one vertex of $C$ or $a_{1}^{\prime}, a_{1}^{\prime \prime}$ and two adjacent vertices of $C$. Since this yields a contradiction to (b) of Lemma 3.2 we conclude the odd cycle containing $a_{1}, a_{1}^{\prime}$ is a triangle. Let $a_{1}^{\prime \prime}$ be the third vertex of this triangle and assume $a_{1}^{\prime \prime} \notin B^{*}$. If $a_{1}^{\prime \prime}$ has a vertex of attachment in $C$ then we can argue as previously to obtain a contradiction to (a) of Lemma 3.2. Hence $a_{1}^{\prime \prime}$ is the cutvertex of two odd cycles, one of which is the triangle containing $a_{1}, a_{1}^{\prime}$. Let $a_{1}^{\prime \prime \prime}$ be a vertex of the other odd cycle, adjacent to $a_{1}^{\prime \prime}$, which is not a cutvertex of $G-V(C)$. Since $a_{1}^{\prime \prime \prime}$ has a vertex of attachment in $C$ we can argue as before to obtain a contradiction to Lemma 3.2. If $a_{1}^{\prime \prime \prime}$ attaches to $d$ or $f$ then we obtain a contradiction to (a) of Lemma 3.2. If $a_{1}^{\prime \prime \prime}$ attaches to any other vertex of $C$ we obtain a contradiction to (b) of Lemma 3.2. We conclude that $a_{1}^{\prime \prime} \in B^{*}$. Hence the graph of $G$ must be as in Figure 4 . The cycle $C$ is denoted by the dashed lines. It is easy to see that $G-E(C)$ is 2-connected.

The other possibility for the positions of $d, f$ in $C$ is $d=a_{2}, f=a_{3}$. We denote the vertices of $C$ in order as $a_{2}, a_{2}^{\prime}, a_{4}, a_{3}, a_{3}^{\prime}, a_{5}$. We consider the vertex $a_{4}$. If the edge $a_{4} a_{2}$ exists we get a contradiction to (b) of Lemma 3.2. Similarly if the edge $a_{4} a_{3}^{\prime}$ exists we have a contradiction. Hence $a_{4}$ has a vertex of attachment in $G-V(C)$. By Lemma 4.1 it cannot be $a_{1}$ or $a_{1}^{\prime}$. Hence it must be the same vertex of attachment of $f$ in $B^{*}$. Otherwise we have a contradiction to (a) of Lemma 3.2. 


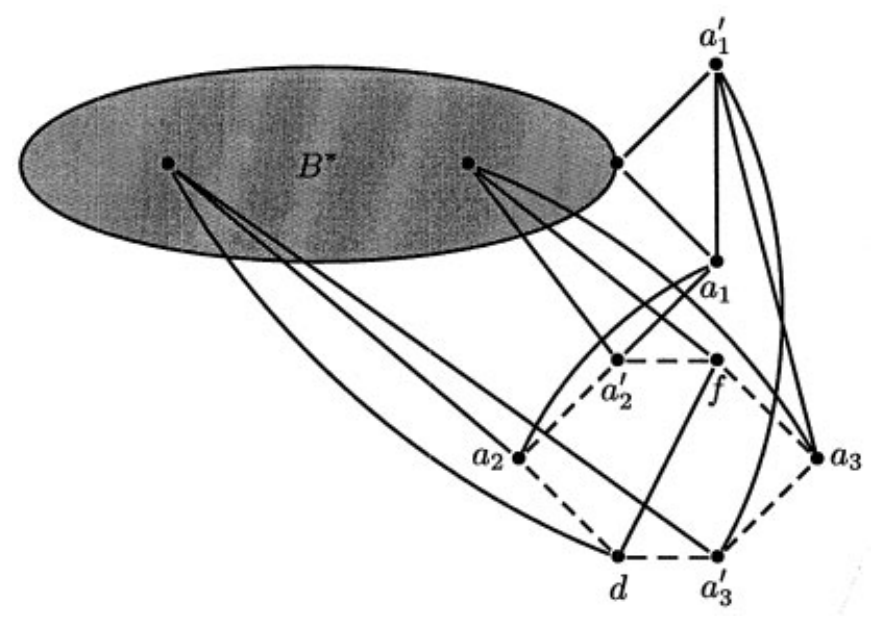

FIGURE 4.

Similarly $a_{5}$ has the same vertex of attachment in $B^{*}$ as $d$. This now puts us in the case we already considered.

Lemma 5.4. Suppose an arc of $C$ joining $d$ to $f$ has order 3 or 2. Then $G-E(C)$ is 2-connected.

Proof. We assume first that an arc of $C$ joining $d$ to $f$ has order 3 and that not both $d, f$ are vertices of attachment of $a_{1}, a_{1}^{\prime}$ in $C$. We can assume wlog that $f=a_{3}$ and the vertices of $C$ in order are $a_{2}, d, a_{3}^{\prime}, a_{3}, a_{4}, a_{2}^{\prime}$. Consider the vertex $a_{4}$. If either of the edges $a_{4} a_{2}$ or $a_{4} a_{3}^{\prime}$ exist then we can obtain a contradiction to (b) of Lemma 3.2. Hence $a_{4}$ has a vertex of attachment in $G-V(C)$ different from $a_{1}, a_{1}^{\prime}$. To avoid a contradiction to (a) of Lemma 3.2 we see this vertex of attachment must be the same as the vertex of attachment of $f$ in $B^{*}$. This puts us into the situation considered in Lemma 5.3.

Let us assume next that the arc of $C$ joining $d$ to $f$ has order 2. To avoid a contradiction to (a) of Lemma 3.2 we can assume that $d=a_{2}, f=a_{2}^{\prime}$. We write the vertices of $C$ in order as $a_{2}, a_{4}, a_{3}^{\prime}, a_{3}, a_{5}, a_{2}^{\prime}$. Consider now the vertex $a_{4}$. If the edge $a_{4} a_{3}$ exists we can form the cycle $C^{\prime}$ consisting of the edges $a_{1}^{\prime} a_{3}, a_{3} a_{4}, a_{4} a_{3}^{\prime}, a_{3}^{\prime} a_{1}^{\prime}$. This contradicts (b) of Lemma 3.2 since $a_{2}$ attaches to $a_{1} \in G-V\left(C^{\prime}\right)$. If the edge $a_{4} a_{2}^{\prime}$ exists we form the cycle $C^{\prime}$ consisting of the edges $a_{1} a_{2}^{\prime}, a_{2}^{\prime} a_{4}, a_{4} a_{2}, a_{2} a_{1}$. This again gives a contradiction to (b) of Lemma 3.2 since $a_{3}$ attaches to $a_{1}^{\prime} \in G-V\left(C^{\prime}\right)$. We conclude $a_{4}$ has a vertex of attachment in $G-V(C)$ different from $a_{1}, a_{1}^{\prime}$. In order to avoid a contradiction to (a) of Lemma 3.2, we see that this vertex of attachment must be the same as the vertex of attachment of $d$ in $B^{*}$. This puts us into the situation discussed in the previous paragraph.

Finally we assume that an arc of $C$ joining $d$ to $f$ has order 3 and that both $d, f$ are vertices of attachment of $a_{1}, a_{1}^{\prime}$ in $C$. Then we can assume wlog that 
$d=a_{2}^{\prime}, f=a_{3}$. We write the vertices of $C$ in order as $a_{2}, a_{4}, a_{3}^{\prime}, a_{3}, a_{5}, a_{2}^{\prime}$. Consider now the vertex $a_{5}$. If either of the edges $a_{5} a_{2}$ or $a_{5} a_{3}^{\prime}$ exist, we obtain a contradiction to (b) of Lemma 3.2 as in the previous paragraph. Hence $a_{5}$ has a vertex of attachment in $G-V(C)$, which is different from $a_{1}, a_{1}^{\prime}$. To avoid a contradiction to (a) of Lemma 3.2, $a_{5}$ can have only one vertex of attachment and it must be the same as the vertex of attachment of $d$ in $B^{*}$. Similarly it must be the same as the vertex of attachment of $f$ in $B^{*}$. Since the vertices of attachment of $d$ and $f$ in $B^{*}$ are different, we have a contradiction.

Proof of Proposition 5.1. This follows from the previous lemmas.

\section{6. $B *$ WITH ONE CUTVERTEX IN $G-V(C), C$ OF ORDER 4}

In this section we prove the following.

Proposition 6.1. Suppose $G$ is a 3-connected graph of minimum degree 4 and the cycle $C$ of Lemma 3.2 has order 4 . If $B^{*}$ has exactly one cutvertex in $G-V(C)$ then there is a cycle $C^{\prime}$ in $G$ of order 4 such that $G-V\left(C^{\prime}\right)$ is nonempty and connected, and $G-E\left(C^{\prime}\right)$ is 2-connected.

Let $a$ denote the cutvertex of $B^{*}$ in $G-V(C)$. Since $G$ is 3 -connected there are two vertices $d, f$ of $C$, which attach to distinct vertices of $B^{*}$. We may assume that the vertex of attachment for $d$ is different from $a$ and the vertex of attachment for $f$ is also different from $a$, unless $B^{*}$ happens to be a $K_{2}$.

Since $B^{*}$ has a cutvertex in $G-V(C)$, there are other endblocks of $G-V(C)$ apart from $B^{*}$. We have already seen in Lemma 3.1 that these endblocks must be either an odd cycle or $K_{2}$. We first assume that $G-V(C)$ has an endblock, which is a $K_{2}$. We denote by $a_{1}$ the vertex of the $K_{2}$, which has degree 1 in $G-V(C)$. Since $a_{1}$ has degree at least 4 in $G$, it has at least three vertices of attachment in $C$. We denote these vertices of attachment, in order on $C$, by $a_{2}, a_{3}, a_{4}$. There is a fourth vertex of $C$, which we denote by $\alpha$. The vertex $\alpha$ may or may not attach to $a_{1}$.

Lemma 6.1. Suppose $G-V(C)$ has just two blocks, $B^{*}$ and a $K_{2}$. Then there is a cycle $C^{\prime}$ in $G$ of order 4 such that $G-V\left(C^{\prime}\right)$ is non-empty and connected, and $G-E\left(C^{\prime}\right)$ is 2-connected.

Proof. Note that the second vertex of the $K_{2}$ is the cutvertex $a$ of $B^{*}$ in $G-V(C)$. We consider the situation where all four vertices of $C$ attach to $a_{1}$. Let us first assume that $C$ has no chords, whence every vertex of $C$ attaches to $B^{*}$. Suppose now $d=a_{2}$. Let $C^{\prime}$ be the cycle consisting of the edges $a_{1} a_{3}, a_{3} a_{2}$, $a_{2} \alpha, \alpha a_{1}$. Then $G-V\left(C^{\prime}\right)$ is the union of $B^{*}$ and the vertex $a_{4}$. Since $a_{4}$ attaches to $B^{*}$, we conclude $G-V\left(C^{\prime}\right)$ is connected. One can also easily see that $G-E\left(C^{\prime}\right)$ is 2-connected. Next we assume that $C$ has exactly one chord, which we can take to be the edge $a_{2} a_{4}$. Let $C^{\prime}$ be the cycle consisting of the edges $a_{1} a_{2}, a_{2} a_{4}, a_{4} \alpha, \alpha a_{1}$. Thus $G-V\left(C^{\prime}\right)$ is the union of $B^{*}$ and the vertex $a_{3}$. Since 
$a_{3}$ has degree at least 4 , it has an edge to $B^{*}$, whence $G-V\left(C^{\prime}\right)$ is connected. Suppose now $d=\alpha$. Then we can form the path consisting of the edges $a a_{1}, a_{1} a_{4}, a_{4} a_{3}, a_{3} a_{2}, a_{2} \alpha, \alpha d^{*}$, where $d^{*} \neq a$ is the vertex of attachment of $d$ in $B^{*}$. None of these edges are edges of the cycle $C^{\prime}$. We conclude that $G-E\left(C^{\prime}\right)$ is 2-connected. The alternative to $d=\alpha$ is that $\alpha$ attaches to the cutvertex $a$ of $B^{*}$. In that case, $d$ must be one of the vertices $a_{2}, a_{3}, a_{4}$. It is easy to see that no matter which vertex $d$ is, the graph $G-E\left(C^{\prime}\right)$ is 2-connected. Finally we assume that $C$ has two chords, whence the graph on the vertices of $C$ is $K_{4}$. Hence we may form a cycle $C^{\prime}$ on the vertices of $C$ such that $d$ and $f$ are adjacent on $C^{\prime}$. It is clear that $G-E\left(C^{\prime}\right)$ is 2-connected.

We consider the situation where the vertex $\alpha$ of $C$ does not attach to $a_{1}$. Suppose $\alpha$ has two edges to $B^{*}$ and let $C^{\prime}$ be the cycle consisting of the edges $a_{1} a_{2}, a_{2} a_{3}, a_{3} a_{4}, a_{4} a_{1}$. Then $G-V\left(C^{\prime}\right)$ is 2-connected and strictly larger than $B^{*}$, yielding a contradiction to (a) of Lemma 3.2. We conclude that the edge $\alpha a_{3}$ must exist. Suppose the edge $a_{2} a_{4}$ does not exist. Then the vertices $a_{2}, a_{4}, \alpha$ attach to $B^{*}$. Let $C^{\prime}$ be the cycle consisting of the edges $a_{1} a_{2}, a_{2} a_{3}, a_{3} a_{4}, a_{4} a_{1}$. Since $\alpha$ attaches to $B^{*}$, it follows that $G-V\left(C^{\prime}\right)$ is connected. One can also see that $G-E\left(C^{\prime}\right)$ is 2-connected by considering the possible positions of $d \in V(C)$. Finally we assume $C$ has two chords. If $d$ and $f$ are adjacent on $C$ then it is easy to see that $G-E(C)$ is 2-connected. Suppose then $d$ and $f$ are non-adjacent on $C$ with $d=\alpha$, whence $f=a_{3}$. Let $C^{\prime}$ be the cycle consisting of the edges $a_{1} a_{2}$, $a_{2} a_{4}, a_{4} a_{3}, a_{3} a_{1}$. Then $G-V\left(C^{\prime}\right)$ is connected and $G-E\left(C^{\prime}\right)$ is 2-connected. One similarly sees that if $d=a_{3}, f=\alpha$, then $G-V\left(C^{\prime}\right)$ is connected and $G-E\left(C^{\prime}\right)$ is 2-connected. The only alternative left is to suppose $d=a_{2}, f=a_{4}$ and $\alpha$ attaches to $a \in B^{*}$. Let $C^{\prime}$ be the cycle consisting of the edges $a_{1} a_{2}, a_{2} a_{3}$, $a_{3} a_{4}, a_{4} a_{1}$. Then $G-V\left(C^{\prime}\right)$ is connected and $G-E\left(C^{\prime}\right)$ is 2-connected.

Lemma 6.2. Suppose $G-V(C)$ has an endblock which is a $K_{2}$ and that $a_{1}$ is the vertex of the $K_{2}$ which has degree 1 in $G-V(C)$. Assume all four vertices of $C$ attach to $a_{1}$. Then there is a cycle $C^{\prime}$ in $G$ of order 4 such that $G-V\left(C^{\prime}\right)$ is non-empty and connected, and $G-E\left(C^{\prime}\right)$ is 2-connected.

Proof. Suppose first that $G-V(C)$ has a second endblock which is a $K_{2}$ and $a_{1}^{\prime}$ is its vertex of degree 1 in $G-V(C)$. Then $a_{1}^{\prime}$ attaches to three vertices of $C$. Suppose one of these vertices is $d$. Let $C^{\prime}$ be the cycle with vertices $a_{1}$ and the three vertices of $C$ different from $d$. Since $d$ attaches to a vertex of $B^{*}$ different from its cutvertex in $G-V(C)$, it follows that $G-V\left(C^{\prime}\right)$ has a block which strictly contains $B^{*}$, contradicting (a) of Lemma 3.2. If $d$ is not a vertex of attachment for $a_{1}^{\prime}$ in $C$ then we can form the cycle $C^{\prime \prime}$ consisting of $a_{1}^{\prime}$ and the three vertices of attachment of $a_{1}^{\prime}$ in $C$. Again we see that $G-V\left(C^{\prime \prime}\right)$ has a block strictly containing $B^{*}$. We conclude that $G-V(C)$ has only one endblock, which is a $K_{2}$.

Next suppose $G-V(C)$ has an endblock, which is an odd cycle. Let $a_{1}^{\prime}, a_{1}^{\prime \prime}$ be adjacent vertices of the cycle, which are not cutvertices of $G-V(C)$. Note in this 
case that $B^{*}$ cannot be a $K_{2}$, whence $d, f$ attach to distinct vertices of $B^{*}$ different from $a$. If $a_{1}^{\prime}$ or $a_{1}^{\prime \prime}$ attach to $d$ or $f$ then we can repeat the argument of the previous paragraph to obtain a contradiction. Hence both $a_{1}^{\prime}, a_{1}^{\prime \prime}$ attach to the vertices of $C$ different from $d, f$. In that case we can form a cycle $C^{\prime}$ of order 4 from the vertices $a_{1}^{\prime}, a_{1}^{\prime \prime}$ and the vertices of $C$ different from $d, f$. Since $a_{1}$ attaches to $d$, we again get a contradiction to (a) of Lemma 3.2. We conclude $G-V(C)$ does not have an endblock, which is an odd cycle.

We are reduced to the situation where $G-V(C)$ has just two endblocks, one of which is $B^{*}$ and the other the $K_{2}$ with $a_{1}$ as vertex of degree 1 in $G-V(C)$. Let $a_{1}^{\prime}$ be the second vertex of the $K_{2}$. If $a_{1}^{\prime}=a$ we are in the situation of Lemma 6.1 so we shall assume $a_{1}^{\prime} \neq a$. Now $a_{1}^{\prime}$ is a cutvertex in $G-V(C)$ of another block. We shall show this block is also a $K_{2}$ and its second vertex is $a \in B^{*}$. To see this, we proceed by contradiction. Let $a_{1}^{\prime \prime} \neq a$ be a vertex of the block adjacent to $a_{1}^{\prime}$. Then $a_{1}^{\prime \prime}$ has a vertex of attachment in $C$. Suppose $a_{1}^{\prime \prime}$ attaches to $d$ or $f$, and let $C^{\prime}$ be the cycle consisting of $a_{1}$, and the three vertices of $C$ different from the vertex of attachment of $a_{1}^{\prime \prime}$. Then $G-V\left(C^{\prime}\right)$ has a block strictly larger than $B^{*}$, contradicting (a) of Lemma 3.2. If $a_{1}^{\prime \prime}$ attaches to neither $d$ nor $f$, then let $C^{\prime}$ be the cycle consisting of $a_{1}, a_{1}^{\prime}, a_{1}^{\prime \prime}$ and the vertex of attachment of $a_{1}^{\prime \prime}$ in $C$. Again $G-V\left(C^{\prime}\right)$ has a block strictly larger than $B^{*}$, yielding a contradiction.

We are now in the situation where $G-V(C)$ consists of three blocks, namely $B^{*}$ and the two $K_{2}$ 's, $a a_{1}^{\prime}$ and $a_{1}^{\prime} a_{1}$. Evidently $a_{1}^{\prime}$ has two vertices of attachment in $C$. If either of these coincides with $d$ or $f$, we get a contradiction as in the previous paragraph. Hence they do not coincide with $d$ or $f$. If $d$ and $f$ are adjacent on $C$, let $C^{\prime}$ be the cycle consisting of $a_{1}, a_{1}^{\prime}$ and the two vertices of $C$ different from $d, f$. Then $G-V\left(C^{\prime}\right)$ contains a block larger than $B^{*}$, a contradiction. Hence $d$ and $f$ are non-adjacent on $C$, whence we get the situation in Figure 5 . It is clear that $G-E(C)$ is 2-connected.

Lemma 6.3. Suppose $G-V(C)$ has an endblock which is a $K_{2}$. If $C$ has two chords then $G-V(C)$ has just two blocks.

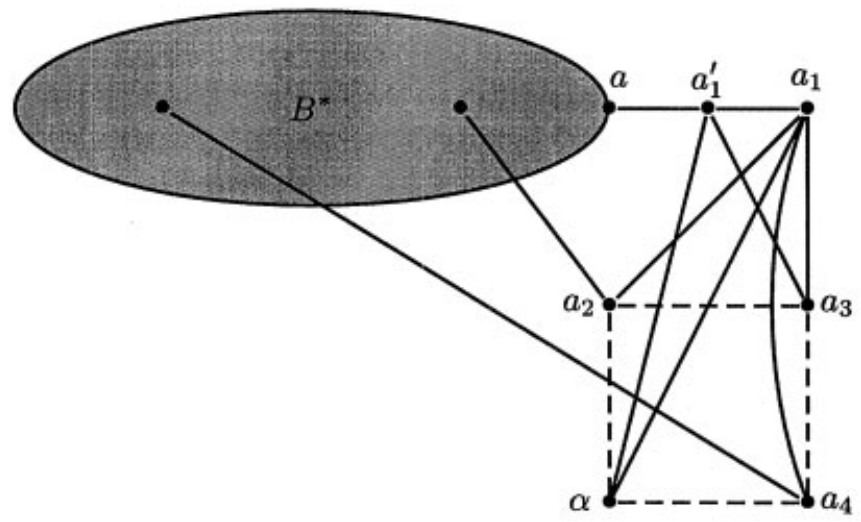

FIGURE 5. 
Proof. We first show that $G-V(C)$ has only two endblocks, $B^{*}$ and the $K_{2}$ with $a_{1}$ as vertex. To see this, suppose there is a third endblock which is a $K_{2}$ and let $a_{1}^{\prime}$ be its vertex of degree 1 in $G-V(C)$. Then $a_{1}^{\prime}$ has three vertices of attachment in $C$, whence it must attach to $d$ or $f$, say $d$. One can construct a cycle $C^{\prime}$ from $a_{1}$ and the three vertices of $C$ other than $d$. It is clear that $G-V\left(C^{\prime}\right)$ contains a block strictly larger than $B^{*}$, yielding a contradiction to (a) of Lemma 3.2. We conclude the third endblock cannot be a $K_{2}$. Suppose then that it is an odd cycle. Let $a_{1}^{\prime}, a_{1}^{\prime \prime}$ be adjacent vertices of the odd cycle, which are not cutvertices of $G-V(C)$. If $a_{1}^{\prime}$ or $a_{1}^{\prime \prime}$ attach to $d$ or $f$ then we can argue as above to obtain a contradiction to (a) of Lemma 3.2. Hence $a_{1}^{\prime}$ and $a_{1}^{\prime \prime}$ both attach to the two vertices of $C$ different from $d, f$. Let $C^{\prime}$ be the cycle with vertices $a_{1}^{\prime}, a_{1}^{\prime \prime}$ and the two vertices of $C$ other than $d, f$. It is clear that $G-V\left(C^{\prime}\right)$ contains a block strictly larger than $B^{*}$, again a contradiction. We conclude that $G-V(C)$ has only two endblocks.

Finally let us assume the second vertex of the $K_{2}$ which is an endblock of $G-V(C)$ is $a_{1}^{\prime} \neq a$. Now $a_{1}^{\prime}$ is a cutvertex for exactly two blocks. Suppose the other block is also a $K_{2}$. Then $a_{1}^{\prime}$ has two vertices of attachment in $C$. If $a_{1}^{\prime}$ attaches to $d$ or $f$, we can argue as in the previous paragraph to obtain a contradiction. Hence $a_{1}^{\prime}$ attaches to two vertices $v, w$ of $C$ different from $d, f$. One of these vertices, say $v$, must attach to $a_{1}$. Let $C^{\prime}$ be the cycle consisting of the edges $a_{1} v, v w, w a_{1}^{\prime}, a_{1}^{\prime} a_{1}$. Note that since the graph on $V(C)$ is $K_{4}$ the edges $v w$ and $d f$ exist. It is clear therefore that $G-V\left(C^{\prime}\right)$ contains a block strictly larger than $B^{*}$, again a contradiction. Suppose the other block with cutvertex $a_{1}^{\prime}$ is an odd cycle. Then there is a vertex $a_{1}^{\prime \prime}$ of this cycle, adjacent to $a_{1}^{\prime}$, which is not a cutvertex of $G-V(C)$. Hence $a_{1}^{\prime \prime}$ has two vertices of attachment in $C$. We can assume as above that neither of these vertices of attachment is $d$ or $f$. Hence $a_{1}^{\prime \prime}$ attaches to a vertex $v$ of $C$, different from $d$ and $f$, which is also a vertex of attachment for $a_{1}$. Let $C^{\prime}$ be the cycle consisting of the edges $a_{1} v, v a_{1}^{\prime \prime}, a_{1}^{\prime \prime} a_{1}^{\prime}, a_{1}^{\prime} a_{1}$. Then $G-V\left(C^{\prime}\right)$ contains a block strictly larger than $B^{*}$, whence we have a contradiction. We conclude the second vertex of the endblock $K_{2}$ is $a$.

Lemma 6.4. Suppose $G-V(C)$ has an endblock which is a $K_{2}$ and the vertex $a_{1}$ of degree 1 in $G-V(C)$ does not attach to a vertex $\alpha$ of $C$. Suppose further that $\alpha$ is not the vertex of a chord of $C$. Then $d$ and $f$ must coincide with $a_{2}$ and $a_{4}$.

Proof. We first observe that $d$ or $f$ cannot coincide with $\alpha$. To see this, let $C^{\prime}$ be the cycle consisting of the edges $a_{1} a_{2}, a_{2} a_{3}, a_{3} a_{4}, a_{4} a_{1}$. Now $\alpha$ has two edges to $G-V\left(C^{\prime}\right)$. If one of these attaches to a vertex of $B^{*}$ other than $a$, then there is a block of $G-V\left(C^{\prime}\right)$ strictly larger than $B^{*}$, contradicting (a) of Lemma 3.2.

Next we show that $d$ and $f$ cannot be adjacent vertices of $C$. We proceed by contradiction. Hence let us assume $d=a_{2}, f=a_{3}$. We first show that the only endblocks of $G-V(C)$ are $B^{*}$ and the $K_{2}$ with $a_{1}$ as vertex. To see this suppose there is a third endblock which is a $K_{2}$ and its vertex of degree 1 in $G-V(C)$ is 
$a_{1}^{\prime}$. Suppose $a_{1}^{\prime}$ attaches to $a_{3}$. Let $C^{\prime}$ be the cycle consisting of the edges $a_{1} a_{2}, a_{2} \alpha, \alpha a_{4}, a_{4} a_{1}$. Then $G-V\left(C^{\prime}\right)$ contains a block strictly larger than $B^{*}$, contradicting (a) of Lemma 3.2. Hence the vertices of attachment of $a_{1}^{\prime}$ are $a_{2}, \alpha, a_{4}$. We can now form the cycle $C^{\prime}$ consisting of the edges $a_{1}^{\prime} a_{2}, a_{2} \alpha, \alpha a_{4}$, $a_{4} a_{1}^{\prime}$. Again we see that $G-V\left(C^{\prime}\right)$ contains a block strictly larger than $B^{*}$, a contradiction. We conclude $G-V(C)$ does not have a third endblock, which is a $K_{2}$. Suppose there is a third endblock which is an odd cycle. Let $a_{1}^{\prime}, a_{1}^{\prime \prime}$ be adjacent vertices of the cycle, which are not cutvertices of $G-V(C)$. As in the previous argument we see that neither $a_{1}^{\prime}$ nor $a_{1}^{\prime \prime}$ can attach to $a_{3}$. Now one can see that it is possible to form an even cycle $C^{\prime}$ from four of the vertices $a_{1}^{\prime}, a_{1}^{\prime \prime}, a_{2}, \alpha, a_{4}$. In that case, $G-V\left(C^{\prime}\right)$ contains a block strictly larger than $B^{*}$, a contradiction.

We are now in the situation where the only endblocks of $G-V(C)$ are $B^{*}$ and the $K_{2}$ with $a_{1}$ as vertex. We have already observed that $\alpha$ has two edges to $G-V(C)$ and that the only vertex of $B^{*}$ it can attach to is the cutvertex $a$ of $B^{*}$ in $G-V(C)$. Hence $\alpha$ attaches to two vertices $a_{1}^{\prime}, a_{1}^{\prime \prime}$ of $G-V(C)$ with $a_{1}^{\prime} \notin B^{*}$ and $a_{1}^{\prime \prime}=a$ if $a_{1}^{\prime \prime} \in B^{*}$. Furthermore, there are paths $P\left(a_{1}^{\prime}, a_{1}\right)$ from $a_{1}^{\prime}$ to $a_{1}$ and $P\left(a_{1}^{\prime \prime}, a\right)$ from $a_{1}^{\prime \prime}$ to $a$ in $G-V(C)$, which do not intersect and only intersect $B^{*}$ in the vertex $a$. Suppose the path $P\left(a_{1}^{\prime}, a_{1}\right)$ has an even number of vertices, and let $C^{\prime}$ be the even cycle consisting of the edges $a_{4} \alpha, a_{4} a_{1}, \alpha a_{1}^{\prime}$ and the path $P\left(a_{1}^{\prime}, a_{1}\right)$. Then $G-V\left(C^{\prime}\right)$ contains a block strictly larger than $B^{*}$, a contradiction. Hence $P\left(a_{1}^{\prime}, a_{1}\right)$ has an odd number of vertices. We show that $\alpha$ is the only vertex of attachment of $a_{1}^{\prime}$ in $C$. To see this, first note that $a_{3}$ cannot be a vertex of attachment for $a_{1}^{\prime}$ by the argument of the previous paragraph. Suppose now $a_{2}$ is a vertex of attachment for $a_{1}^{\prime}$. Let $C^{\prime}$ be the cycle consisting of the edges $a_{2} a_{1}, a_{2} a_{1}^{\prime}$ and the path $P\left(a_{1}^{\prime}, a_{1}\right)$, whence $C^{\prime}$ is an even cycle. Let $f^{*} \neq a$ be the vertex of attachment of $f$ in $B^{*}$. Since the path consisting of the edges $f^{*} a_{3}, a_{3} a_{4}, a_{4} \alpha, \alpha a_{1}^{\prime \prime}$ and $P\left(a_{1}^{\prime \prime}, a\right)$ is disjoint from $C^{\prime}$, it follows that $G-V\left(C^{\prime}\right)$ contains a block strictly larger than $B^{*}$, again a contradiction. A similar argument yields a contradiction if $a_{1}^{\prime}$ attaches to $a_{4}$.

We are now in the situation where the path $P\left(a_{1}^{\prime}, a_{1}\right)$ has an odd number of vertices and the only vertex of attachment in $C$ for $a_{1}^{\prime}$ is $\alpha$. It follows that $a_{1}^{\prime}$ is a cutvertex of $G-V(C)$ and one of the two blocks with cutvertex $a_{1}^{\prime}$ is an odd cycle. Let $a_{1}^{\prime \prime \prime}$ be a vertex of this cycle adjacent to $a_{1}^{\prime}$, which is not a cutvertex of $G-V(C)$. Then $a_{1}^{\prime \prime \prime}$ has two vertices of attachment in $C$. If $a_{1}^{\prime \prime \prime}$ attaches to $a_{3}$ then we get a contradiction as in the previous paragraph. If $a_{1}^{\prime \prime \prime}$ attaches to $\alpha$ then we again get a contradiction since there is a path from $a_{1}^{\prime \prime \prime}$ to $a_{1}$, which has an even number of vertices. We conclude that $a_{1}^{\prime \prime \prime}$ attaches to both $a_{2}$ and $a_{4}$. Let $C^{\prime}$ be the cycle consisting of the edges $\alpha a_{4}, a_{4} a_{1}^{\prime \prime \prime}, a_{1}^{\prime \prime \prime} a_{1}^{\prime}, a_{1}^{\prime} \alpha$. Then $G-V\left(C^{\prime}\right)$ contains a block strictly larger than $B^{*}$, contradicting (a) of Lemma 3.2. We conclude that $d$ and $f$ are not adjacent vertices of $C$ and neither of them coincides with $\alpha$.

Lemma 6.5. Suppose $G-V(C)$ has an endblock which is a $K_{2}$ and the vertex $a_{1}$ of degree 1 in $G-V(C)$ does not attach to a vertex $\alpha$ of $C$. Suppose further that $\alpha$ is not the vertex of a chord of $C$. Then 
(1) There are two endblocks of $G-V(C)$ which are $K_{2}$ 's. Suppose $a_{1}^{\prime}$ is the vertex of degree 1 in $G-V(C)$ of the second $K_{2}$. Then the vertices of attachment of $a_{1}^{\prime}$ in $C$ are $a_{2}, \alpha, a_{4}$.

(2) Any endblock of $G-V(C)$ other than $B^{*}$ which is an odd cycle is a triangle. The vertices of attachment in $C$ of the non-cutvertices of the triangle are precisely $a_{2}, a_{4}$.

(3) $G-V(C)$ has at most two cutvertices. If a is the only cutvertex then $a_{3}, \alpha$ attach to $a$.

(4) If $G-V(C)$ has a second cutvertex $a_{1}^{\prime \prime}$, then $a_{1}^{\prime \prime}$ attaches to both $\alpha$ and $a_{3}$ in $C$. The only blocks of $G-V(C)$ with cutvertex a are $B^{*}$ and the $K_{2}, a_{1}^{\prime \prime} a$ with vertices $a_{1}^{\prime \prime}$ and $a$.

Proof. By Lemma 6.4 we can assume that $d=a_{2}, f=a_{4}$. Further, if $\alpha$ attaches to a vertex of $B^{*}$ it must be the cutvertex $a$ of $B^{*}$ in $G-V(C)$.

We show first that there is at most one other endblock which is a $K_{2}$ and that the vertices of attachment of its vertex $a_{1}^{\prime}$ of degree 1 in $G-V(C)$ satisfy (1) in the statement of the lemma. To see this, suppose such a $K_{2}$ exists and $a_{3}$ is a vertex of attachment for $a_{1}^{\prime}$. Then $a_{1}^{\prime}$ also attaches to either $a_{2}$ or $a_{4}$, say $a_{2}$. Let $C^{\prime}$ be the cycle consisting of the edges $a_{1} a_{2}, a_{2} a_{1}^{\prime}, a_{1}^{\prime} a_{3}, a_{3} a_{1}$. Since $\alpha$ does not attach to $a_{1}$, it attaches to a vertex of $G-V\left(C^{\prime}\right)$. Hence $G-V\left(C^{\prime}\right)$ contains a block strictly larger than $B^{*}$, a contradiction. We conclude the vertices of attachment of $a_{1}^{\prime}$ in $C$ are precisely $a_{2}, \alpha, a_{4}$. A similar argument shows there can be no further endblocks which are $K_{2}$ 's.

Next we show that if there is an endblock other than $B^{*}$, which is an odd cycle, it must be a triangle. Further, the vertices of attachment in $C$ of the noncutvertices of the triangle satisfy (2) in the statement of the lemma. To see this, let $a_{1}^{\prime}, a_{1}^{\prime \prime}$ be adjacent vertices of the odd cycle, which are not cutvertices of $G-V(C)$. Suppose $a_{2}$ is not a vertex of attachment for either $a_{1}^{\prime}$ or $a_{1}^{\prime \prime}$. Then we can form an even cycle $C^{\prime}$ from four of the vertices $a_{1}^{\prime}, a_{1}^{\prime \prime}, a_{3}, a_{4}, \alpha$. Since $d=a_{2}$, $G-V\left(C^{\prime}\right)$ contains a block strictly larger than $B^{*}$, a contradiction. We conclude $a_{2}$ is a vertex of attachment for $a_{1}^{\prime}$ or $a_{1}^{\prime \prime}$ and similarly also $a_{4}$. It is not hard to see now that $a_{2}, a_{4}$ are the only vertices of attachment in $C$ of $a_{1}^{\prime}, a_{1}^{\prime \prime}$, and the cycle containing $a_{1}^{\prime}, a_{1}^{\prime \prime}$ is a triangle.

We consider the cutvertices of $G-V(C)$. Let us suppose $G-V(C)$ has more than one cutvertex. Then there is a cutvertex $a_{1}^{\prime \prime} \notin B^{*}$ with the property that the connected components of $G-V(C)-\left\{a_{1}^{\prime \prime}\right\}$ consist of the component containing $B^{*}$, and components $B-\left\{a_{1}^{\prime \prime}\right\}$, where $B$ is an endblock of $G-V(C)$ with cutvertex $a_{1}^{\prime \prime}$. Suppose one of the endblocks $B$ with cutvertex $a_{1}^{\prime \prime}$ is a triangle. Then we can form a cycle $C^{\prime}$ of order 4 from the vertices of the triangle and $a_{2}$. To avoid a contradiction to (a) of Lemma 3.2, it follows that both $\alpha$ and $a_{3}$ attach to $a_{1}^{\prime \prime}$. Further, there are two endblocks, of $G-V(C)$ which are $K_{2}$ 's and their cutvertex in $G-V(C)$ is $a_{1}^{\prime \prime}$.

Suppose none of the endblocks $B$ with cutvertex $a_{1}^{\prime \prime}$ are triangles. Consider the situation where there is just one endblock $B$ with cutvertex $a_{1}^{\prime \prime}$. Then $B$ is a $K_{2}$, 
which we can assume wlog is the $K_{2}, a_{1}^{\prime \prime} a_{1}$. Further, $a_{1}^{\prime \prime}$ has a vertex of attachment $\beta$ in $C$. If $\beta=a_{3}$, we let $C^{\prime}$ be the cycle with edges $a_{1} a_{2}, a_{2} a_{3}, a_{3} a_{1}^{\prime \prime}, a_{1}^{\prime \prime} a_{1}$. Since $\alpha$ has two vertices of attachment in $G-V(C)$, it follows that $G-V\left(C^{\prime}\right)$ contains a block strictly larger than $B^{*}$. Since this contradicts (a) of Lemma 3.2, we conclude $a_{3}$ does not attach to $a_{1}^{\prime \prime}$. Hence $a_{3}$ attaches to a vertex of $G-V(C)-$ $\left\{a_{1}, a_{1}^{\prime \prime}\right\}$. Let $C^{\prime}$ be the cycle with vertices $a_{1}, a_{1}^{\prime \prime}, \beta$, and a vertex of $C$ adjacent to $\beta$, which is also a vertex of attachment for $a_{1}$. Evidently $V\left(C^{\prime}\right)$ does not contain both $a_{2}, a_{4}$, or both $\alpha, a_{3}$. Hence $G-V\left(C^{\prime}\right)$ contains a block larger than $B^{*}$, contradicting (a) of Lemma 3.2. We conclude there cannot be just one endblock $B$ with cutvertex $a_{1}^{\prime \prime}$. It follows that the $K_{2}, a_{1}^{\prime} a_{1}^{\prime \prime}$ exists. We may therefore form the cycle $C^{\prime}$ with edges $a_{2} a_{1}, a_{1} a_{1}^{\prime \prime}, a_{1}^{\prime \prime} a_{1}^{\prime}, a_{1}^{\prime} a_{2}$. Since $G-V\left(C^{\prime}\right)$ cannot contain a block larger than $B^{*}$, we see that both $\alpha$ and $a_{3}$ attach to $a_{1}^{\prime \prime}$.

We have then that the endblocks $B$ of $G-V(C)$ with cutvertex $a_{1}^{\prime \prime}$ consist of triangles and the $K_{2}$ 's $a_{1}^{\prime \prime} a_{1}, a_{1}^{\prime \prime} a_{1}^{\prime}$. Further $a_{1}^{\prime \prime}$ attaches to both $\alpha$ and $a_{3}$. We can also see that there are exactly two vertices of attachment of $V(C)$ in the connected component of $G-V(C)-\left\{a_{1}^{\prime \prime}\right\}$ containing $B^{*}$. These are the vertices of attachment in $B^{*}$ of $d=a_{2}$ and $f=a_{4}$. It follows that the blocks of $G-V(C)$ are $B^{*}$, the $K_{2}$ 's $a a_{1}^{\prime \prime}, a_{1}^{\prime \prime} a_{1}, a_{1}^{\prime \prime} a_{1}^{\prime}$ and triangles with cutvertex $a_{1}^{\prime \prime}$ in $G-V(C)$. We have proved (1) to (4) in the case $G-V(C)$ has more than one cutvertex.

We finally deal with the situation where $G-V(C)$ has just one cutvertex $a$. Since the vertices of attachment in $C$ for the non-cutvertices of blocks, which are triangles are $a_{3}, a_{4}$, we conclude that a second block which is a $K_{2}$ must exist. This $K_{2}$ is $a_{1}^{\prime} a$ and $a_{1}^{\prime}$ attaches to $\alpha$. Since $\alpha, a_{3}$ have degree at least 4 , they must also attach to $a \in B^{*}$. We have again verified (1) to (4).

Corollary 6.1. Suppose $G-V(C)$ has an endblock which is a $K_{2}$ and the vertex $a_{1}$ of degree 1 in $G-V(C)$ does not attach to a vertex $\alpha$ of $C$. Suppose further that $\alpha$ is not the vertex of a chord of $C$. Then there is cycle $C^{\prime}$ of order 4 in $G$ such that $G-V\left(C^{\prime}\right)$ is non-empty and connected, and $G-E\left(C^{\prime}\right)$ is 2-connected.

Proof. This follows from Lemma 6.5. Let $C^{\prime}$ be the cycle consisting of the edges $a_{1} a_{2}, a_{2} a_{1}^{\prime}, a_{1}^{\prime} a_{4}, a_{4} a_{1}$. It follows from Lemma 6.5 that $G-V\left(C^{\prime}\right)$ is connected and $G-E\left(C^{\prime}\right)$ is 2-connected. We have illustrated this in Figure 6 in the case $G-V(C)$ has two cutvertices. The cycle $C^{\prime}$ has dashed edges.

Lemma 6.6. Suppose $G-V(C)$ has an endblock which is a $K_{2}$ and the vertex $a_{1}$ of degree 1 in $G-V(C)$ does not attach to a vertex $\alpha$ of $C$. Suppose further that $C$ has exactly one chord and $\alpha$ is a vertex of this chord. If the vertices $d, f$ of $C$ do not coincide with $a_{2}, a_{4}$ then $G-V(C)$ consists of two blocks.

Proof. We first consider the case where $d, f$ are adjacent and one of them, say $d$, coincides with $\alpha$. We may assume then that $d=\alpha, f=a_{2}$. Consider a vertex $\beta$ of $G-V(C)$, which has the property $\beta \neq a_{1}, \beta \notin B^{*}$. Then $\beta$ can attach only to $a_{3}$ or $a_{4}$ in $C$. Otherwise we obtain a contradiction to (a) of Lemma 3.2. It follows that any endblock of $G-V(C)$, other than the $K_{2}$ with vertex $a_{1}$ and $B^{*}$, must be 


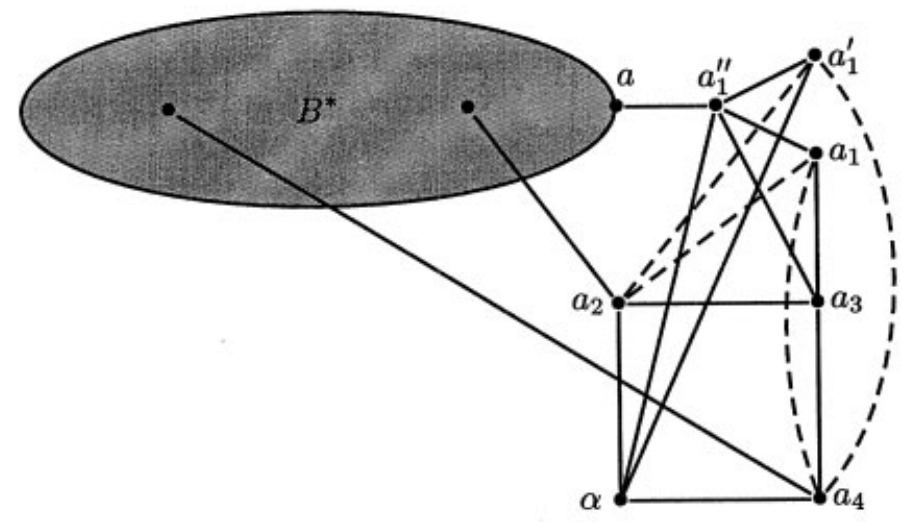

FIGURE 6.

an odd cycle. Let $a_{1}^{\prime}, a_{1}^{\prime \prime}$ be adjacent vertices of such an odd cycle, which are not cutvertices of $G-V(C)$. Then $a_{1}^{\prime}, a_{1}^{\prime \prime}$ attach to both $a_{3}$ and $a_{4}$. Hence we can form the cycle $C^{\prime}$ of order 4 consisting of the edges $a_{1}^{\prime} a_{3}, a_{3} a_{4}, a_{4} a_{1}^{\prime \prime}, a_{1}^{\prime \prime} a_{1}^{\prime}$. Evidently $G-V\left(C^{\prime}\right)$ contains a block strictly larger than $B^{*}$, contradicting (a) of Lemma 3.2.

We conclude the only endblocks of $G-V(C)$ are $B^{*}$ and the $K_{2}$ with vertex $a_{1}$. Suppose now the second vertex of this $K_{2}$ is $a_{1}^{\prime} \notin B^{*}$. Then $a_{1}^{\prime}$ must have a vertex of attachment in $C$, which must be either $a_{3}$ or $a_{4}$. Hence we can form a cycle $C^{\prime}$ with vertices $a_{1}, a_{1}^{\prime}, a_{3}, a_{4}$. Again $G-V\left(C^{\prime}\right)$ contains a block strictly larger than $B^{*}$, contradicting (a) of Lemma 3.2. We conclude the second vertex of the $K_{2}$ is $a \in B^{*}$, whence $G-V(C)$ has two blocks.

Next we consider the case where $d, f$ are adjacent but neither coincides with $\alpha$. Hence we may assume $d=a_{2}, f=a_{3}$. By the argument of the previous paragraph, we see that a vertex $\beta$ of $G-V(C), \beta \neq a_{1}, \beta \notin B^{*}$ can attach only to $a_{4}$ or $\alpha$ in $C$. Hence any endblock of $G-V(C)$, other than the $K_{2}$ with vertex $a_{1}$ and $B^{*}$, must be an odd cycle. Arguing as before, we see that the only endblocks of $G-V(C)$ are $B^{*}$ and the $K_{2}$ with vertex $a_{1}$. Suppose now the second vertex of this $K_{2}$ is $a_{1}^{\prime} \notin B^{*}$. Then $a_{1}^{\prime}$ has a vertex of attachment in $C$, which must be either $\alpha$ or $a_{4}$. If $\alpha$ is a vertex of attachment, then we may argue as in the previous paragraph to obtain a contradiction to (a) of Lemma 3.2. Hence $a_{1}^{\prime}$ attaches to $a_{4}$ and does not attach to $\alpha$. Since $a_{1}^{\prime}$ attaches only to $a_{4}$ in $C$ the second block with cutvertex $a_{1}^{\prime}$ is an odd cycle. Let $a_{1}^{\prime \prime}$ be a vertex of this cycle adjacent to $a_{1}^{\prime}$, which is not a cutvertex of $G-V(C)$. Then $a_{1}^{\prime \prime}$ attaches to both $\alpha$ and $a_{4}$. Let $C^{\prime}$ be the cycle consisting of the edges $a_{4} a_{1}, a_{1} a_{1}^{\prime}, a_{1}^{\prime} a_{1}^{\prime \prime}, a_{1}^{\prime \prime} a_{4}$. Then $G-V\left(C^{\prime}\right)$ contains a block strictly larger than $B^{*}$, contradicting (a) of Lemma 3.2. In all cases we have a contradiction, whence $G-V(C)$ consists of two blocks.

Finally we consider the case where $d, f$ are non-adjacent, whence we may assume $d=\alpha, f=a_{3}$. As previously we see that a vertex $\beta$ of $G-V(C)$, $\beta \neq a_{1}, \beta \notin B^{*}$ can attach only to $a_{2}$ or $a_{4}$ in $C$. Hence any endblock of $G-V(C)$, other than the $K_{2}$ with vertex $a_{1}$ and $B^{*}$, must be an odd cycle. 
Suppose there is an endblock which is an odd cycle. Let $a_{1}^{\prime}, a_{1}^{\prime \prime}$ be adjacent vertices of the cycle, which are not cutvertices of $G-V(C)$. Then $a_{1}^{\prime}, a_{1}^{\prime \prime}$ attach to both $a_{2}$ and $a_{4}$. Hence we may form the cycle $C^{\prime}$ consisting of the edges $a_{1}^{\prime} a_{2}, a_{2} a_{1}^{\prime \prime}, a_{1}^{\prime \prime} a_{4}, a_{4} a_{1}^{\prime}$. Since the edge $\alpha a_{3}$ exists, $G-V\left(C^{\prime}\right)$ contains a block strictly larger than $B^{*}$, contradicting (a) of Lemma 3.2. We conclude the only endblocks of $G-V(C)$ are $B^{*}$ and the $K_{2}$ with vertex $a_{1}$. Let $a_{1}^{\prime} \notin B^{*}$ be the second vertex of this $K_{2}$. Then $a_{1}^{\prime}$ has a vertex of attachment in $C$, which must be either $a_{2}$ or $a_{4}$. Suppose $a_{1}^{\prime}$ attaches to both $a_{2}$ and $a_{4}$. Let $C^{\prime}$ be the cycle with edges $a_{1} a_{2}, a_{2} a_{1}^{\prime}, a_{1}^{\prime} a_{4}, a_{4} a_{1}$. Then $G-V\left(C^{\prime}\right)$ contains a block strictly larger than $B^{*}$, contradicting (a) of Lemma 3.2. Next suppose $a_{1}^{\prime}$ attaches to $a_{2}$ but does not attach to $a_{4}$. Then $a_{4}$ attaches to a vertex of $G-V(C)$ other than $a_{1}, a_{1}^{\prime}$. Suppose $d$ attaches to a vertex $d^{*} \in B^{*}$. If the vertex of attachment of $a_{4}$ is different from $d^{*}$ then we can obtain a contradiction to (a) of Lemma 3.2. If the vertex of attachment of $a_{4}$ is $d^{*}$, then this puts us in the situation of adjacent $d, f$ dealt with in the previous paragraphs. We conclude again in this case that $G-V(C)$ contains two blocks.

Lemma 6.7. Suppose $G-V(C)$ has an endblock which is a $K_{2}$, and the vertex $a_{1}$ of degree 1 in $G-V(C)$ does not attach to a vertex $\alpha$ of $C$. Suppose further that $C$ has exactly one chord and $\alpha$ is a vertex of this chord. If the vertices $d, f$ of $C$ coincide with $a_{2}, a_{4}$ then there exists a cycle $C^{\prime}$ in $G$ of order 4 such that $G-V\left(C^{\prime}\right)$ is non-empty and connected, and $G-E\left(C^{\prime}\right)$ is 2-connected.

Proof. Arguing as in the previous lemma, we see that a vertex $\beta$ of $G-V(C), \beta \neq a_{1}, \beta \notin B^{*}$ can attach only to $\alpha$ or $a_{3}$ in $C$. We can similarly see that the only endblocks of $G-V(C)$ are $B^{*}$ and the $K_{2}$ with vertex $a_{1}$. Let $a_{1}^{\prime}$ be the second vertex of this $K_{2}$, and suppose $a_{1}^{\prime} \notin B^{*}$. Then $a_{1}^{\prime}$ is the cutvertex of two blocks. One of these is the $K_{2} a_{1} a_{1}^{\prime}$. Let us assume the other is an odd cycle. Let $a_{1}^{\prime \prime}$ be a vertex of this cycle adjacent to $a_{1}^{\prime}$ which is not a cutvertex of $G-V(C)$. Then $a_{1}^{\prime \prime}$ attaches to both $\alpha$ and $a_{3}$. Let $C^{\prime}$ be the cycle consisting of the edges $a_{3} a_{1}, a_{1} a_{1}^{\prime}, a_{1}^{\prime} a_{1}^{\prime \prime}, a_{1}^{\prime \prime} a_{3}$. Then $G-V\left(C^{\prime}\right)$ contains a block strictly larger than $B^{*}$, contradicting (a) of Lemma 3.2. We conclude $a_{1}^{\prime}$ is the cutvertex of two blocks, both of which are $K_{2}$ 's. Hence $a_{1}^{\prime}$ attaches to both $a_{3}$ and $\alpha$ in $C$.

Consider now the second $K_{2}$ which has cutvertex $a_{1}^{\prime}$ in $G-V(C)$. One of its vertices is $a_{1}^{\prime}$ and denote the other vertex $a_{1}^{\prime \prime}$. Suppose $a_{1}^{\prime \prime} \notin B^{*}$, whence $a_{1}^{\prime \prime}$ has a vertex of attachment in $C$. Suppose $a_{3}$ is this vertex of attachment, and let $C^{\prime}$ be the cycle consisting of the edges $a_{3} a_{1}, a_{1} a_{1}^{\prime}, a_{1}^{\prime} a_{1}^{\prime \prime}, a_{1}^{\prime \prime} a_{3}$, Then $G-V\left(C^{\prime}\right)$ contains a block strictly larger than $B^{*}$, contradicting (a) of Lemma 3.2. The other possibility for the vertex of attachment in $C$ of $a_{1}^{\prime \prime}$ is $\alpha$. Let $C^{\prime}$ be the cycle consisting of the edges $a_{1} a_{2}, a_{2} a_{3}, a_{3} a_{1}^{\prime}, a_{1}^{\prime} a_{1}$. Then $G-V\left(C^{\prime}\right)$ contains a block strictly larger than $B^{*}$, again a contradiction.

We conclude that $G-V(C)$ consists of at most three blocks. One of these is $B^{*}$ and the others are $K_{2}$ 's. If $G-V(C)$ has just two blocks, the conclusion of the lemma follows from Lemma 6.1. Suppose then that $G-V(C)$ has three blocks 
with the endblock, which is a $K_{2}$ having vertices $a_{1}$ of degree 1 in $G-V(C)$ and $a_{1}^{\prime}$ of degree 2 in $G-V(C)$. Now $a_{1}$ attaches to $a_{2}, a_{3}, a_{4}$ in $C$ and $a_{1}^{\prime}$ to $a_{3}, \alpha$. Furthermore, the diagonal $a_{3} \alpha$ exists. Let $C^{\prime}$ be the cycle consisting of the edges $a_{1} a_{2}, a_{2} a_{3}, a_{3} a_{4}, a_{4} a_{1}$. It is clear that $G-V\left(C^{\prime}\right)$ is connected and $G-E\left(C^{\prime}\right)$ is 2connected. We illustrate this in Figure 7.

Corollary 6.2. Suppose $G$ satisfies the conditions of Proposition 6.1 and $G-V(C)$ has an endblock which is a $K_{2}$. Then there is a cycle $C^{\prime}$ in $G$ of order 4 such that $G-V\left(C^{\prime}\right)$ is non-empty and connected, and $G-E\left(C^{\prime}\right)$ is 2-connected.

Proof. This follows from the previous lemmas.

To finish the proof of Proposition 6.1, we may now assume that all endblocks of $G-V(C)$ other than $B^{*}$ are odd cycles. We denote by $a_{1}, a_{1}^{\prime}$ two adjacent vertices of an endblock which is an odd cycle, neither of which is a cutvertex of $G-V(C)$. Hence both $a_{1}$ and $a_{1}^{\prime}$ have two vertices of attachment in $C$. There are two possibilties for these vertices of attachment:

(1) Both $a_{1}$ and $a_{1}^{\prime}$ have precisely two vertices of attachment in $C$ and these are identical for $a_{1}$ and $a_{1}^{\prime}$. Furthermore, the vertices of attachment are non-adjacent on $C$.

(2) There are two adjacent vertices of $C$ which form a cycle of order 4 together with the vertices $a_{1}, a_{1}^{\prime}$.

In case (1) we denote the vertices of attachment of $a_{1}, a_{1}^{\prime}$ in $C$ by $a_{2}, a_{3}$. In case (2) we denote by $a_{2}, a_{3}$ the vertices of $C$ such that the cycle with edges $a_{1} a_{2}, a_{2} a_{3}, a_{3} a_{1}^{\prime}, a_{1}^{\prime} a_{1}$ exists. Now $C$ has two other vertices, which we denote $\alpha, \beta$ with $\alpha$ being the vertex of $C$ adjacent to $a_{2}$. We continue to denote by $d, f$ two vertices of $C$ which have edges to distinct vertices of $B^{*}$ different from the cutvertex $a$ of $B^{*}$ in $G-V(C)$.

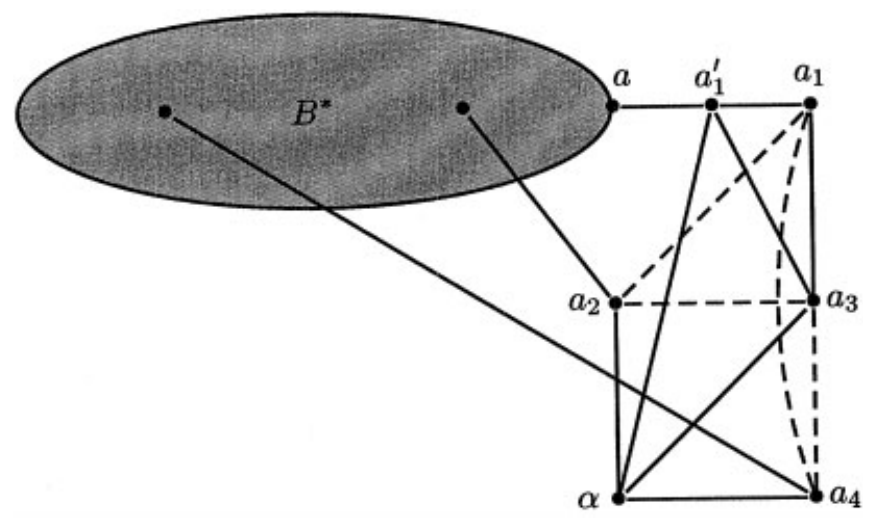

FIGURE 7. 
Lemma 6.8. Suppose all endblocks of $G-V(C)$ other than $B^{*}$ are odd cycles. Let $a_{1}, a_{1}^{\prime}$ be two adjacent vertices of an endblock which is an odd cycle and such that neither $a_{1}$ nor $a_{1}^{\prime}$ is a cutvertex of $G-V(C)$. If $a_{2}$ and $a_{3}$ are non-adjacent on $C$ then there is a cycle $C^{\prime}$ of order 4 in $C$ such that $G-V\left(C^{\prime}\right)$ is connected and $G-E\left(C^{\prime}\right)$ is 2-connected.

Proof. Since $a_{2}$ and $a_{3}$ are non-adjacent we are in case (1) above. Suppose first that $d$ and $f$ are non-adjacent and do not coincide with $a_{2}, a_{3}$. Now $d$ and $f$ do not attach to $a_{1}$ or $a_{1}^{\prime}$. Hence either $d$ attaches to a second vertex of $G-V(C)$ different from $a_{1}, a_{1}^{\prime}$ or the edge $d f$ exists. In either case we obtain a contradiction to (a) of Lemma 3.2. Suppose next that $d=a_{2}, f=a_{3}$. We show the odd cycle containing the vertices $a_{1}, a_{1}^{\prime}$ is a triangle. To see this, assume the cycle is not a triangle, whence we can suppose there is a vertex $a_{1}^{\prime \prime}$ of the cycle, adjacent to $a_{1}^{\prime}$ on the cycle, which is not a cutvertex of $G-V(C)$. Hence $a_{1}^{\prime \prime}$ has two vertices of attachment in $C$. Suppose $a_{1}^{\prime \prime}$ attaches to $\alpha$. Let $C^{\prime}$ be the cycle with edges $a_{1}^{\prime} a_{2}, a_{2} \alpha, \alpha a_{1}^{\prime \prime}, a_{1}^{\prime \prime} a_{1}^{\prime}$. Then $G-V\left(C^{\prime}\right)$ contains a block which includes $B^{*}$ and $a_{3}$, contradicting (a) of Lemma 3.2. Since we may make a similar argument if $a_{1}^{\prime \prime}$ attaches to $\beta$ we conclude that the vertices of attachment in $C$ of $a_{1}^{\prime \prime}$ are precisely $a_{2}, a_{3}$. Since $\alpha$ and $\beta$ have degree at least 4 in $G$, they must attach to a vertex of $G-V(C)$ other than $a_{1}, a_{1}^{\prime}, a_{1}^{\prime \prime}$. Suppose $d$ attaches to the vertex $d^{*}$ in $B^{*}$. By forming the cycle $C^{\prime}$ consisting of the edges $a_{3} a_{1}, a_{1} a_{1}^{\prime}, a_{1}^{\prime} a_{1}^{\prime \prime}, a_{1}^{\prime \prime} a_{3}$ we see that $d^{*}$ can be the only vertex of attachment for $\alpha$ and $\beta$ in $G-V(C)$. Otherwise we get a contradiction to (a) of Lemma 3.2. Since a similar argument implies that the vertex of attachment $f^{*}$ of $f$ in $B^{*}$ is the only vertex of attachment for $\alpha$ and $\beta$ in $G-V(C)$, we arrive at a contradiction. We conclude the odd cycle containing the vertices $a_{1}, a_{1}^{\prime}$ is a triangle. It is not difficult to see further that all endblocks other than $B^{*}$ are triangles and the vertices of attachment in $C$ of their non-cutvertices are precisely $a_{2}, a_{3}$.

We show that the cutvertices of all endblocks other than $B^{*}$ are identical. To see this assume for contradiction this is not the case. Then there exists a cutvertex $a_{1}^{\prime \prime} \notin B^{*}$ with the property that the components of $G-V(C)-\left\{a_{1}^{\prime \prime}\right\}$ consist of the component containing $B^{*}$ and $B-\left\{a_{1}^{\prime \prime}\right\}$ for endblocks $B$ of $G-V(C)$ with cutvertex $a_{1}^{\prime \prime}$. Let one of the endblocks $B$ be the triangle with vertices $a_{1}, a_{1}^{\prime}, a_{1}^{\prime \prime}$ and $C^{\prime}$ be the cycle with edges $a_{2} a_{1}, a_{1} a_{1}^{\prime \prime}, a_{1}^{\prime \prime} a_{1}^{\prime}, a_{1}^{\prime} a_{2}$. Since $G-V(C)$ contains an endblock triangle with cutvertex different from $a_{1}^{\prime \prime}$ it follows that $G-V(C)$ contains a block containing $B^{*}$ and $a_{3}$, contradicting (a) of Lemma 3.2. We conclude that all endblocks of $G-V(C)$ other than $B^{*}$ have the same cutvertex $a_{1}^{\prime \prime}$.

Suppose $a_{1}^{\prime \prime} \notin B^{*}$. Then the blocks of $G-V(C)$ consist of $B^{*}$, the $K_{2}, a a_{1}^{\prime \prime}$ and the endblock triangles with cut vertex $a_{1}^{\prime \prime}$. Otherwise there is a vertex of the component of $G-V(C)-\left\{a_{1}^{\prime \prime}\right\}$ containing $B^{*}$, which attaches to a vertex of $C$. Further, this vertex does not lie in $B^{*}$. By considering the cycle $C^{\prime}$ with vertices $a_{1}, a_{1}^{\prime}, a_{1}^{\prime \prime}$ and one of the vertices $a_{2}$ or $a_{3}$, we see $G-V\left(C^{\prime}\right)$ contains a block strictly larger than $B^{*}$, contradicting (a) of Lemma 3.2. Consider next the vertices 
of attachment in $G-V(C)$ of $\alpha$. If $\alpha$ attaches to a vertex of $B^{*}$, then we can obtain a contradiction to (a) of Lemma 3.2. Hence $\alpha$ attaches to $a_{1}^{\prime \prime}$ and the diagonal $\alpha \beta$ exists. Similarly $\beta$ attaches to $a_{1}^{\prime \prime}$. Let $C^{\prime}$ be the cycle consisting of the edges $a_{1} a_{2}, a_{2} \alpha, \alpha a_{3}, a_{3} a_{1}$. Then $G-V\left(C^{\prime}\right)$ is connected and $G-E\left(C^{\prime}\right)$ is 2-connected. We illustrate this in Figure 8.

Next we assume $a_{1}^{\prime \prime}=a \in B^{*}$. It follows from the previous paragraph that all endblocks are triangles with cutvertex $a$ in $G-V(C)$. Further, the vertices of attachment in $C$ of the non-cutvertices of these triangles are precisely $a_{2}$ and $a_{3}$. Hence $\alpha$ and $\beta$ can attach only outside $C$ to vertices of $B^{*}$. If $\alpha$ attaches to two vertices of $B^{*}$ then we have a contradiction to (a) of Lemma 3.2. In fact if $C^{\prime}$ is the cycle with edges $a_{1} a_{2}, a_{2} \beta, \beta a_{3}, a_{3} a_{1}$ then $G-V\left(C^{\prime}\right)$ contains a block strictly larger than $B^{*}$. We conclude that the edge $\alpha \beta$ exists and that $\alpha$ and $\beta$ attach to the same vertex of $B^{*}$. Note this last assertion is deduced by considering the cycle $C^{\prime}$ consisting of the edges $a_{1} a_{2}, a_{2} a_{1}^{\prime}, a_{1}^{\prime} a_{3}, a_{3} a_{1}$. The situation we are in is illustrated in Figure 9. We have assumed that $\alpha$ and $\beta$ attach to the cutvertex $a$ of $B^{*}$ in $G-V(C)$. Again it is clear that if $C^{\prime}$ is the cycle consisting of the edges $a_{1} a_{2}, a_{2} \alpha, \alpha a_{3}, a_{3} a_{1}$, then $G-V\left(C^{\prime}\right)$ is connected and $G-E\left(C^{\prime}\right)$ is 2-connected.

Finally we consider the situation where $d$ and $f$ are adjacent on $C$. We can assume wlog that $d=a_{2}, f=\alpha$. Now $\alpha$ does not attach to $a_{1}$ or $a_{1}^{\prime}$. Hence if $\alpha$ attaches to two vertices of $G-V(C)$, we can obtain a contradiction to (a) of Lemma 3.2. It follows that the edge $\alpha \beta$ exists and that $\alpha$ and $\beta$ have the same vertex of attachment in $G-V(C)$. Arguing as previously, we see that the odd cycle containing the vertices $a_{1}, a_{1}^{\prime}$ is a triangle and its third vertex is $a \in B^{*}$. It is also easy to see that all endblocks are triangles with cutvertex $a \in B^{*}$. Furthermore, the vertices of attachment in $C$ of the non-cutvertices are precisely $a_{2}$ and $a_{3}$. Let $C^{\prime}$ be the cycle with edges, $a_{1} a_{2}, a_{2} \alpha, \alpha a_{3}, a_{3} a_{1}$. We see that $G-V\left(C^{\prime}\right)$ is connected and $G-E\left(C^{\prime}\right)$ is 2-connected. This is illustrated in Figure 10.

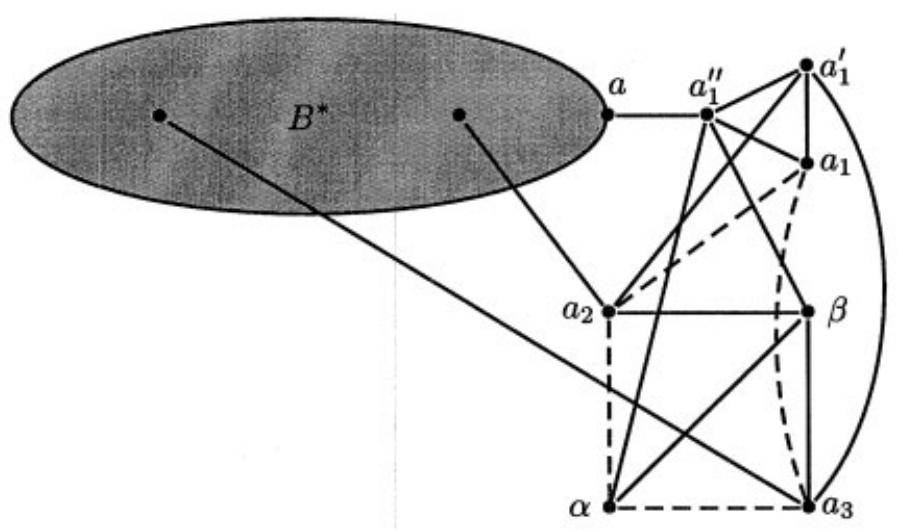

FIGURE 8. 


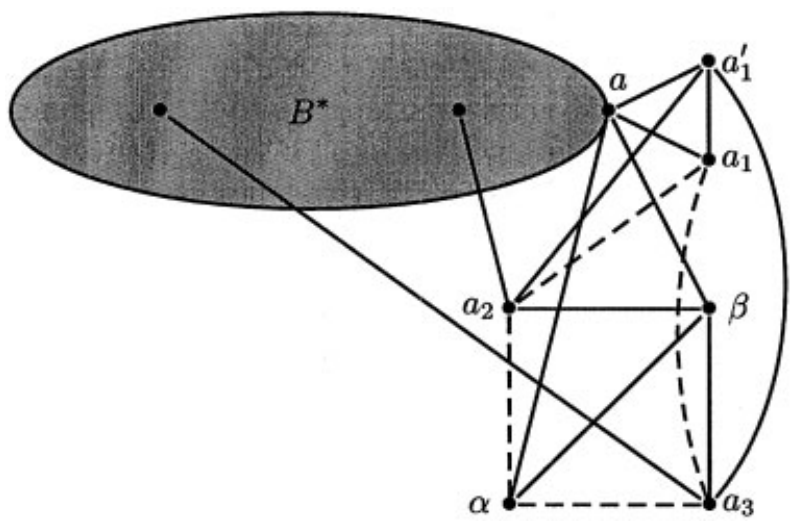

FIGURE 9.

Lemma 6.9. Suppose all endblocks of $G-V(C)$ other than $B^{*}$ are odd cycles. Let $a_{1}$ be a vertex of an endblock which is an odd cycle and assume $a_{1}$ is not a cutvertex of $G-V(C)$. Then $a_{1}$ has exactly two vertices of attachment in $C$.

Proof. Evidently $a_{1}$ has at least two vertices of attachment in $C$. Let us first suppose $a_{1}$ has four vertices of attachment in $C$, whence $a_{1}$ attaches to every vertex of $C$. Let $a_{1}^{\prime}$ be a vertex of the endblock odd cycle adjacent to $a_{1}$, which is also not a cutvertex of $G-V(C)$. If $a_{1}^{\prime}$ attaches to $d$ or $f$ in $C$ then we obtain a contradiction to (a) of Lemma 3.2. Hence $a_{1}^{\prime}$ must attach to the two vertices of $C$ different from $d, f$. In that case we also get a contradiction to (a) of Lemma 3.2. In fact if $d, f$ are adjacent on $C$, we can construct the cycle $C^{\prime}$ with vertices $a_{1}, a_{1}^{\prime}$ and the two vertices of $C$ distinct from $d, f$. Then $G-V\left(C^{\prime}\right)$ contains a block strictly larger than $B^{*}$. If $d, f$ are non-adjacent on $C$ we can construct a cycle $C^{\prime}$ with vertices $a_{1}^{\prime}, f$ and the two vertices of attachment of $a_{1}^{\prime}$ in $C$. Again $G-V\left(C^{\prime}\right)$ contains a block strictly larger than $B^{*}$. We conclude that $a_{1}$ cannot have four vertices of attachment in $C$.

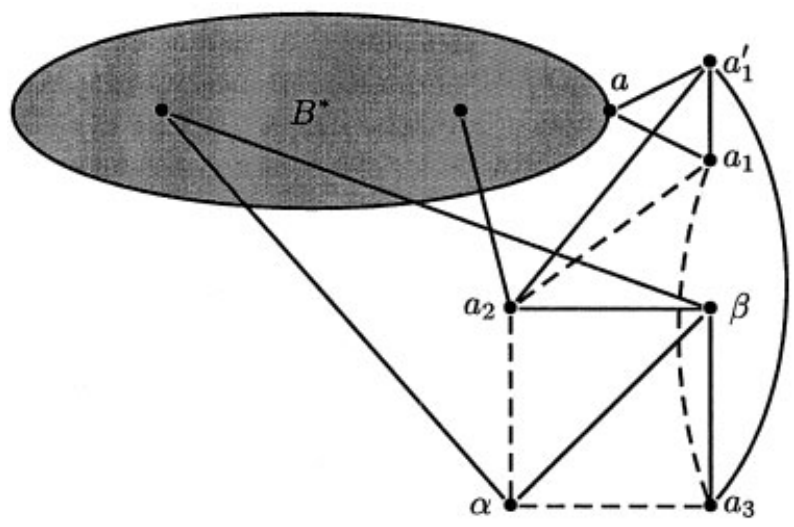

FIGURE 10. 
Suppose $a_{1}$ has three vertices of attachment in $C$. We denote these vertices in order on $C$ by $a_{2}, a_{3}, a_{4}$ and denote by $\alpha$ the remaining vertex of $C$, which does not attach to $a_{1}$. As in the previous paragraph, let $a_{1}^{\prime}$ be a vertex on the odd cycle adjacent to $a_{1}$, which is not a cutvertex of $G-V(C)$. Suppose that $a_{1}^{\prime}$ attaches to $\alpha$. Then $d, f$ cannot coincide with $\alpha$ or we would have a contradiction to (a) of Lemma 3.2. Assume $a_{3}$ is the second vertex of attachment for $a_{1}^{\prime}$ in $C$. Then $d, f$ cannot coincide with $a_{3}$ either, whence we may assume $d=a_{2}, f=a_{4}$. Let $C^{\prime}$ be the cycle with edges $a_{1}^{\prime} \alpha, \alpha a_{4}, a_{4} a_{3}, a_{3} a_{1}^{\prime}$. Then $G-V\left(C^{\prime}\right)$ contains a block strictly larger than $B^{*}$, contradicting (a) of Lemma 3.2. Hence $a_{3}$ cannot be the second vertex of attachment for $a_{1}^{\prime}$ in $C$. We can assume then that $a_{2}$ is the second vertex of attachment for $a_{1}^{\prime}$. If $d$ and $f$ are adjacent on $C$, it is easy to see we get a contradiction to (a) of Lemma 3.2. Hence we may assume $d=a_{2}, f=a_{4}$. Suppose now the diagonal $a_{3} \alpha$ exists. Let $C^{\prime}$ be the cycle consisting of the edges $a_{1}^{\prime} \alpha, \alpha a_{3}, a_{3} a_{2}, a_{2} a_{1}^{\prime}$. Then $G-V\left(C^{\prime}\right)$ contains a block strictly larger than $B^{*}$, contradicting (a) of Lemma 3.2. Hence the diagonal $a_{3} \alpha$ does not exist, in which case $\alpha$ attaches to a vertex of $G-V(C)$ different from $a_{1}, a_{1}^{\prime}$. We have already seen that if $\alpha$ attaches to a vertex of $B^{*}$, it has to be the cutvertex of $B^{*}$ in $G-V(C)$. In particular, it cannot be the same as the vertex of attachment in $B^{*}$ of $f=a_{4}$. Let $C^{\prime}$ be the cycle consisting of the edges $a_{1}^{\prime} a_{2}, a_{2} a_{3}, a_{3} a_{1}, a_{1} a_{1}^{\prime}$. Then $G-V\left(C^{\prime}\right)$ contains a block strictly larger than $B^{*}$, again a contradiction to (a) of Lemma 3.2.

We conclude now that $a_{1}^{\prime}$ does not attach to $\alpha$. Then $\alpha$ attaches to a vertex of $G-V(C)$ different from $a_{1}, a_{1}^{\prime}$. Suppose first $\alpha=d$. Then, to avoid a contradiction to (a) of Lemma 3.2, $\alpha$ can have only one vertex of attachment in $G-V(C)$. Hence the diagonal $a_{3} \alpha$ exists. Consider now the position of $f$ on $C$. If $f$ is adjacent to $\alpha$, then one can construct a cycle $C^{\prime}$ with vertices $a_{1}, a_{1}^{\prime}$ and the two vertices of $C$ different from $d, f$. In that case, $G-V\left(C^{\prime}\right)$ contains a block strictly larger than $B^{*}$, contradicting (a) of Lemma 3.2. Hence we must have $f=a_{3}$. Observe that $a_{1}^{\prime}$ cannot attach to $a_{3}$. If it did, we could form the cycle $C^{\prime}$ from the edges $a_{1} a_{2}, a_{2} \alpha, \alpha a_{4}, a_{4} a_{1}$, and $G-V\left(C^{\prime}\right)$ contains a block strictly larger than $B^{*}$. We conclude the vertices of attachment in $C$ of $a_{1}^{\prime}$ are $a_{2}, a_{4}$. In that case, let $C^{\prime}$ be the cycle with the edges $a_{1} a_{2}, a_{2} a_{1}^{\prime}, a_{1}^{\prime} a_{4}, a_{4} a_{1}$. Since the diagonal $a_{3} \alpha$ exists, $G-V\left(C^{\prime}\right)$ contains a block strictly larger than $B^{*}$, contracting (a) of Lemma 3.2.

We can assume now that if the vertex of attachment of $\alpha$ in $G-V(C)$ lies in $B^{*}$, it is the cutvertex of $B^{*}$ in $G-V(C)$. Hence one of $d, f$ coincides with one of $a_{2}, a_{4}$, say $d=a_{2}$. In that case, we can form a cycle $C^{\prime}$ with vertices $a_{1}, a_{1}^{\prime}, a_{3}, a_{4}$, since $a_{1}^{\prime}$ attaches to $a_{3}$ or $a_{4}$. Then $G-V\left(C^{\prime}\right)$ contains a block strictly larger than $B^{*}$, again a contradiction to (a) of Lemma 3.2. It follows that $a_{1}$ cannot have three vertices of attachment in $C$.

Lemma 6.10. Suppose all endblocks of $G-V(C)$ other than $B^{*}$ are odd cycles. Let $a_{1}, a_{1}^{\prime}$ be two adjacent vertices of an endblock which is an odd cycle, and such that neither $a_{1}$ nor $a_{1}^{\prime}$ is a cutvertex of $G-V(C)$. Suppose $a_{1}$ attaches to a vertex 
$a_{2}$ of $C$ and $a_{1}^{\prime}$ to a vertex $a_{3}$ of $C$, and that $a_{2}, a_{3}$ are adjacent on $C$. If $d, f$ are not adjacent on $C$, then $G-E(C)$ is 2-connected.

Proof. Let $\alpha, \beta$ be the remaining two vertices of $C$ different from $a_{2}, a_{3}$, with $\alpha$ adjacent to $a_{2}$. We can assume wlog that $d=a_{2}, f=\beta$. By Lemma 6.9 we have that $a_{1}$ has exactly two vertices of attachment in $C$, one of which is $a_{2}$. Similarly $a_{1}^{\prime}$ has two vertices of attachment, one of which is $a_{3}$. Suppose that $a_{1}^{\prime}$ attaches to $\alpha$. Let $C^{\prime}$ be the cycle consisting of the edges $a_{1}^{\prime} a_{3}, a_{3} \beta, \beta \alpha, \alpha a_{1}^{\prime}$. Since $d=a_{2}$, the graph $G-V\left(C^{\prime}\right)$ contains a block strictly larger than $B^{*}$, contradicting (a) of Lemma 3.2. Hence $a_{1}^{\prime}$ cannot attach to $\alpha$. Suppose next that $a_{1}$ attaches to $\beta$. Then $\alpha$ has a vertex of attachment in $G-V(C)$, which is different from $a_{1}, a_{1}^{\prime}$. Let $C^{\prime}$ be the cycle with edges $a_{1} a_{2}, a_{2} a_{3}, a_{3} a_{1}^{\prime}, a_{1}^{\prime} a_{1}$. To avoid a contradiction to (a) of Lemma 3.2, we must have that the vertex of attachment of $\alpha$ in $G-V(C)$ is the same as the vertex of attachment in $B^{*}$ of $f=\beta$. By similar argument, we see the vertex of attachment of $\alpha$ must also be the same as the vertex of attachment in $B^{*}$ of $d=a_{2}$. Since the vertices of attachment in $B^{*}$ of $d, f$ are different, we have a contradiction.

We conclude the vertices of attachment of $a_{1}$ in $C$ are adjacent, and similarly with $a_{1}^{\prime}$. Suppose now that $a_{3}$ is the second vertex of attachment in $C$ for $a_{1}$ and $\beta$ is the second vertex of attachment for $a_{1}^{\prime}$. By arguing as in the previous paragraph, we see that the vertex of attachment of $\alpha$ in $G-V(C)$ must coincide with the vertex of attachment in $B^{*}$ of $d$ and also of $f$, a contradiction. Suppose next that $\alpha$ is the second vertex of attachment in $C$ for $a_{1}$, and $a_{2}$ is the second vertex of attachment for $a_{1}^{\prime}$. Observe that since $\beta$ cannot have two vertices of attachment in $G-V(C)$, the diagonal $a_{2} \beta$ exists. If the diagonal $\alpha a_{3}$ exists, let $C^{\prime}$ be the cycle consisting of the edges $a_{1} \alpha, \alpha a_{3}, a_{3} a_{1}^{\prime}, a_{1}^{\prime} a_{1}$. Then $G-V\left(C^{\prime}\right)$ contains a block strictly larger than $B^{*}$, contradicting (a) of Lemma 3.2. We conclude the edge $\alpha a_{3}$ does not exist, whence $\alpha$ has a vertex of attachment in $G-V(C)$ different from $a_{1}, a_{1}^{\prime}$. By considering the cycle $C^{\prime}$ with edges $a_{1} a_{2}, a_{2} a_{3}, a_{3} a_{1}^{\prime}, a_{1}^{\prime} a_{1}$, we see that this vertex of attachment for $\alpha$ must be the same as the vertex of attachment for $f$ in $B^{*}$. Otherwise we have a contradiction to (a) of Lemma 3.2. Now let $C^{\prime}$ be the cycle with edges $a_{1}^{\prime} a_{2}, a_{2} \beta, \beta a_{3}, a_{3} a_{1}^{\prime}$. Then $G-V\left(C^{\prime}\right)$ contains a block strictly larger than $B^{*}$, again a contradiction.

We are left with two possibilities for the second vertices of attachment for $a_{1}, a_{1}^{\prime}$ in $C$ :

(1) $a_{1}$ attaches to $a_{3}, a_{1}^{\prime}$ attaches to $a_{2}$;

(2) $a_{1}$ attaches to $\alpha, a_{1}^{\prime}$ attaches to $\beta$.

Neither of (1), (2) lead to contradictions, but they do yield a situation where the cycle $C$ satisfies the conclusion of the lemma. We first consider (1).

In case (1) we see that the diagonals $\alpha a_{3}$ and $a_{2} \beta$ exist and that $\alpha, \beta$ have both exactly one vertex of attachment in $G-V(C)$, which is the vertex of attachment for $f=\beta \in B^{*}$. Otherwise we would have a contradiction to (a) of Lemma 3.2. Next we show that the odd cycle containing the vertices $a_{1}, a_{1}^{\prime}$ is a triangle with 
cutvertex $a \in B^{*}$. To see this let $a_{1}^{\prime \prime}$ be a vertex of the cycle adjacent to $a_{1}$ or $a_{1}^{\prime}$, which is not a cutvertex of $G-V(C)$. Arguing as before, we see that the vertices of attachment in $C$ of $a_{1}^{\prime \prime}$ must be precisely $a_{2}, a_{3}$. Hence we can form the cycle $C^{\prime}$ consisting of the edges $a_{3} a_{1}, a_{1} a_{1}^{\prime}, a_{1}^{\prime} a_{1}^{\prime \prime}, a_{1}^{\prime \prime} a_{3}$, and $G-V\left(C^{\prime}\right)$ contains a block strictly larger than $B^{*}$, a contradiction to (a) of Lemma 3.2. We conclude the odd cycle is a triangle. A similar argument shows its cutvertex in $G-V(C)$ is $a \in B^{*}$. It is not difficult to see further that every endblock of $G-V(C)$, other than $B^{*}$, is a triangle with cutvertex $a \in B^{*}$. Futhermore, the vertices of attachment in $C$ of the non-cutvertices of the triangle are $a_{2}, a_{3}$. It follows now that $G-E(C)$ is 2connected. We illustrate this in Figure 11.

In case (2) we first see that the diagonal $\alpha a_{3}$ cannot exist. If it did, we could form the cycle $C^{\prime}$ consisting of the edges $a_{1} \alpha, \alpha a_{3}, a_{3} a_{2}, a_{2} a_{1}$. Then $G-V\left(C^{\prime}\right)$ contains a block strictly larger than $B^{*}$, a contradiction to (a) of Lemma 3.2. Hence $\alpha$ has a vertex of attachment in $G-V(C)$ different from $a_{1}, a_{1}^{\prime}$. By previous argument, we see this vertex of attachment must coincide with the vertex of attachment in $B^{*}$ of $f=\beta$. Now, arguing as earlier in the paragraph, we see the diagonal $a_{2} \beta$ cannot exist. We also can see that $a_{3}$ has a vertex of attachment in $G-V(C)$ different from $a_{1}, a_{1}^{\prime}$. This vertex of attachment must coincide with the vertex of attachment in $B^{*}$ of $d=a_{2}$. We have now accounted for all edges from $C$ to $G-V(C)$. If another edge existed we would have a contradiction to (a) of Lemma 3.2. It follows that $G-V(C)$ consists of two blocks, $B^{*}$ and a triangle containing the vertices $a_{1}, a_{1}^{\prime}$. The third vertex of the triangle is $a \in B^{*}$. It is easy to see that $G-E(C)$ is 2-connected, as illustrated in Figure 12.

Lemma 6.11. Suppose all endblocks of $G-V(C)$ other than $B^{*}$ are odd cycles. Let $a_{1}, a_{1}^{\prime}$ be two adjacent vertices of an endblock which is an odd cycle, and such that neither $a_{1}$ nor $a_{1}^{\prime}$ is a cutvertex of $G-V(C)$. Suppose $a_{1}$ attaches to a vertex $a_{2}$ of $C$ and $a_{1}^{\prime}$ to a vertex $a_{3}$ of $C$, and that $a_{2}, a_{3}$ are adjacent on $C$. If $d, f$ are adjacent on $C$, then $G-E(C)$ is 2-connected.

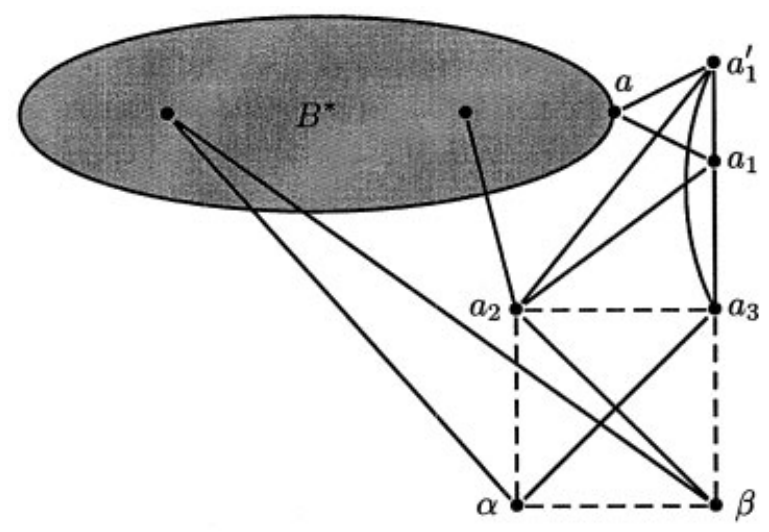

FIGURE 11. 


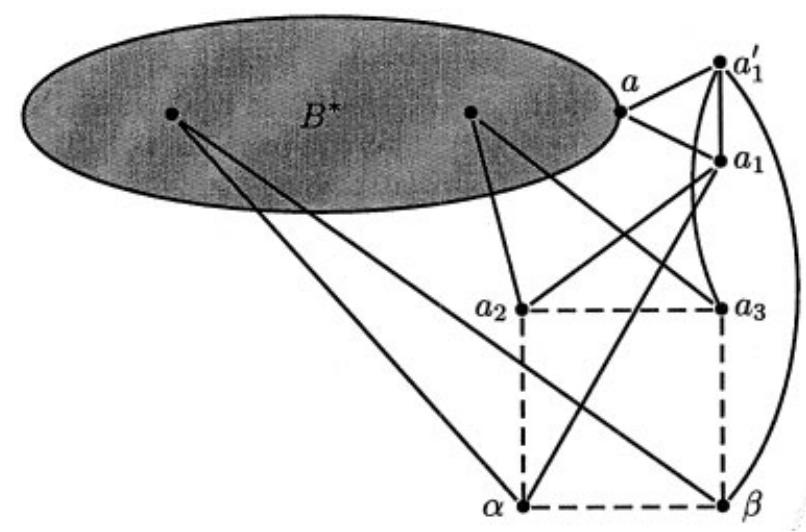

FIGURE 12.

Proof. There are two possible configurations for $d, f$ on $C,(1) d=a_{2}, f=$ $\alpha$, (2) $d=a_{2}, f=a_{3}$. We consider (1) first. Suppose $a_{1}^{\prime}$ attaches to $\alpha$, and let $C^{\prime}$ be the cycle consisting of the edges $a_{1}^{\prime} a_{3}, a_{3} \beta, \beta \alpha, \alpha a_{1}^{\prime}$. Then $G-V\left(C^{\prime}\right)$ contains a block strictly larger than $B^{*}$, contradicting (a) of Lemma 3.2. Suppose $a_{1}$ attaches to $\beta$, and let $C^{\prime}$ be the cycle consisting of the edges $a_{1} \beta, \beta a_{3}, a_{3} a_{1}^{\prime}, a_{1}^{\prime} a_{1}$. Then $G-V\left(C^{\prime}\right)$ contains a block strictly larger than $B^{*}$, again a contradiction. If $a_{1}$ attaches to $a_{3}$ and $a_{1}^{\prime}$ to $\beta$ we similarly get a contradiction. Hence the only possibilities for the second vertices of attachment for $a_{1}, a_{1}^{\prime}$ in $C$ are:

(I) $a_{1}$ attaches to $a_{3}, a_{1}^{\prime}$ to $a_{2}$,

(II) $a_{1}$ attaches to $\alpha, a_{1}^{\prime}$ to $\beta$,

(III) $a_{1}$ attaches to $\alpha, a_{1}^{\prime}$ to $a_{2}$.

We consider the three cases, first (I). Now $\beta$ must have a vertex of attachment in $G-V(C)$ different from $a_{1}, a_{1}^{\prime}$. To avoid a contradiction to (a) of Lemma 3.2 this vertex of attachment must be the same as the vertex of attachment of $f=\alpha$ in $B^{*}$. This puts us in the situation of non-adjacent $d, f$ dealt with in the previous lemma. For case (II) we show first that the edge $a_{2} \beta$ cannot exist. If it did, we could form the cycle $C^{\prime}$ consisting of the edges $a_{1}^{\prime} \beta, \beta a_{2}, a_{2} a_{3}, a_{3} a_{1}^{\prime}$, and $G-V\left(C^{\prime}\right)$ then contains a block strictly larger than $B^{*}$, a contradiction to (a) of Lemma 3.2. Since the edge $a_{2} \beta$ does not exist, $\beta$ has a vertex of attachment in $G-V(C)$ different from $a_{1}, a_{1}^{\prime}$. To avoid a contradiction to (a) of Lemma 3.2, we see that it must be the same as the vertex of attachment of $f=\alpha$ in $B^{*}$. This again puts us in the situation of non-adjacent $d, f$. For case (III) we see that $\beta$ must have a vertex of attachment in $G-V(C)$ different from $a_{1}, a_{1}^{\prime}$. As before, this vertex of attachment must be the same as the vertex of attachment of $f=\alpha$ in $B^{*}$. Hence we are in the situation of non-adjacent $d, f$ again.

Finally we consider the second possible configuration of $d, f$, (2) $d=a_{2}$, $f=a_{3}$. Suppose $a_{1}$ attaches to $\beta$ and let $C^{\prime}$ be the cycle with edges $a_{1} a_{2}$, 
$a_{2} \alpha, \alpha \beta, \beta a_{1}$. Then $G-V\left(C^{\prime}\right)$ contains a block strictly larger than $B^{*}$, contradicting (a) of Lemma 3.2. Hence $a_{1}$ cannot attach to $\beta$, and similarly $a_{1}^{\prime}$ cannot attach to $\alpha$. Suppose now $a_{1}$ attaches to $\alpha$ and $a_{1}^{\prime}$ to $\beta$. Then we may form the cycle $C^{\prime}$ with edges $a_{1} \alpha, \alpha \beta, \beta a_{1}^{\prime}, a_{1}^{\prime} a_{1}$. Since $G-V\left(C^{\prime}\right)$ contains a block strictly larger than $B^{*}$, we have a contradiction. Hence the only possibilities for the second vertices of attachment for $a_{1}, a_{1}^{\prime}$ in $C$ are:

(I) $a_{1}$ attaches to $a_{3}, a_{1}^{\prime}$ to $\beta$,

(II) $a_{1}$ attaches to $\alpha, a_{1}^{\prime}$ to $a_{2}$,

(III) $a_{1}$ attaches to $a_{3}, a_{1}^{\prime}$ to $a_{2}$.

Cases (I) and (II) are similar, so we shall just deal with (I). In that case, $\alpha$ must attach to a vertex of $G-V(C)$ different from $a_{1}, a_{1}^{\prime}$. To avoid a contradiction to (a) of Lemma 3.2, this vertex of attachment must be the same as the vertex of attachment of $d=a_{2}$ in $B^{*}$. This puts us once more in the situation of nonadjacent $d, f$ dealt with in Lemma 6.10.

We are left to deal with case (III). We first show that the odd cycle containing the vertices $a_{1}, a_{1}^{\prime}$ is a triangle. To see this, suppose the odd cycle is not a triangle, in which case there is a vertex $a_{1}^{\prime \prime}$ adjacent to $a_{1}$ or $a_{1}^{\prime}$ which is not cutvertex of $G-V(C)$. We can assume wlog that $a_{1}^{\prime \prime}$ is adjacent to $a_{1}^{\prime}$. If $a_{1}^{\prime \prime}$ attaches to $\alpha$ then we can form the cycle $C^{\prime}$ with edges $a_{1}^{\prime} a_{2}, a_{2} \alpha, \alpha a_{1}^{\prime \prime}, a_{1}^{\prime \prime} a_{1}^{\prime}$. Evidently $G-V\left(C^{\prime}\right)$ contains a block strictly larger than $B^{*}$, contradicting (a) of Lemma 3.2. Since we get a similar contradiction if $a_{1}^{\prime \prime}$ attaches to $\beta$, we conclude $a_{1}^{\prime \prime}$ attaches to both $a_{2}$ and $a_{3}$. Further, $\alpha$ attaches to a vertex of $G-V(C)$ different from $a_{1}, a_{1}^{\prime}, a_{1}^{\prime \prime}$. By forming the cycle $C^{\prime}$ with edges $a_{3} a_{1}, a_{1} a_{1}^{\prime}, a_{1}^{\prime} a_{1}^{\prime \prime}, a_{1}^{\prime \prime} a_{3}$, we see the vertex of attachment of $\alpha$ in $G-V(C)$ must be the same as the vertex of attachment of $d=a_{2}$ in $B^{*}$. Otherwise we contradict (a) of Lemma 3.2. By forming the cycle $C^{\prime}$ with edges $a_{2} a_{1}, a_{1} a_{1}^{\prime}, a_{1}^{\prime} a_{1}^{\prime \prime}, a_{1}^{\prime \prime} a_{2}$, we see the vertex of attachment of $\alpha$ in $G-V(C)$ must be the same as the vertex of attachment of $f=a_{3}$ in $B^{*}$. Since the vertices of attachment of $d, f$ are different, we have a contradiction. We conclude the odd cycle containing the vertices $a_{1}, a_{1}^{\prime}$ is a triangle. It is not difficult to see further that every endblock of $G-V(C)$, other than $B^{*}$, is a triangle. The vertices of attachment in $C$ of the non-cutvertices of the triangle are precisely $a_{2}, a_{3}$.

Let $a_{1}^{\prime \prime}$ be a vertex of attachment in $G-V(C)$ of $\alpha$, and suppose $a_{1}^{\prime \prime} \notin B^{*}$. If $a_{1}^{\prime \prime}$ is not the cutvertex of every endblock of $G-V(C)$ other than $B^{*}$, then there is an endblock $B \neq B^{*}$ such that $a_{1}^{\prime \prime}$ is in the same component of $G-V(C)-V(B)$ as $B^{*}$. From the previous paragraph, we see that we can form a cycle $C^{\prime}$ from the vertices of $B$ and $a_{3}$. Since $G-V\left(C^{\prime}\right)$ contains a block strictly larger than $B^{*}$, we have a contradiction to (a) of Lemma 3.2. We conclude that $a_{1}^{\prime \prime}$ is the cutvertex of every endblock of $G-V(C)$ other than $B^{*}$. In particular, the third vertex of the triangle with vertices $a_{1}, a_{1}^{\prime}$ is $a_{1}^{\prime \prime}$. Note that if a vertex of $G-V(C)$ which does not belong to an endblock (including $B^{*}$ ) attaches to $C$, then we can obtain a contradiction to (a) of Lemma 3.2. Hence the blocks of $G-V(C)$ are $B^{*}$, the $K_{2}$, $a a_{1}^{\prime \prime}$ with $a \in B^{*}$, and triangles with cutvertex $a_{1}^{\prime \prime}$ in $G-V(C)$. Further, $a_{1}^{\prime \prime}$ is 
the only vertex of attachment for $\alpha$ in $G-V(C)$, and hence the diagonal $\alpha a_{3}$ exists. Similarly, $a_{1}^{\prime \prime}$ is the only vertex of attachment for $\beta$ in $G-V(C)$, and hence the diagonal $\beta a_{2}$ exists. It is easy to see now that $G-E(C)$ is 2-connected. We illustrate this in Figure 13.

Suppose finally that $\alpha$ attaches to a vertex of $B^{*}$. If this vertex is different from the cutvertex $a$ of $B^{*}$ in $G-V(C)$, then we are in a situation dealt with previously in this lemma or in Lemma 6.10. Hence we may assume $\alpha$ attaches to $a \in B^{*}$. In that case it is easy to see that the cutvertex of every endblock of $G-V(C)$ is $a$. Further, the diagonal $\alpha a_{3}$ exists. Similarly $\beta$ attaches to $a$ and the diagonal $\beta a_{2}$ exists. We see as above that $G-E(C)$ is 2-connected, as illustrated in Figure 14.

Corollary 6.3. Suppose $G$ satisfies the conditions of Proposition 6.1 and all endblocks of $G-V(C)$ other than $B^{*}$ are odd cycles. Then there is a cycle $C^{\prime}$ in $G$ of order 4 such that $G-V\left(C^{\prime}\right)$ is non-empty and connected, and $G-E\left(C^{\prime}\right)$ is 2connected.

Proof. This follows from Lemmas 6.8 through 6.11 .

Proof of Proposition 6.1. This follows from Corollaries 6.2, 6.3.

\section{7. $B *$ WITH TWO CUTVERTICES IN $G-V(C), C$ OF ORDER 4}

Proposition 7.1. Suppose $G$ is a 3-connected graph of minimum degree 4 and the cycle $C$ of Lemma 3.2 has order 4 . If $B^{*}$ has two cutvertices in $G-V(C)$ then there is a cycle $C^{\prime}$ in $G$ of order 4 such that $G-V\left(C^{\prime}\right)$ is non-empty and connected, and $G-E\left(C^{\prime}\right)$ is 2-connected.

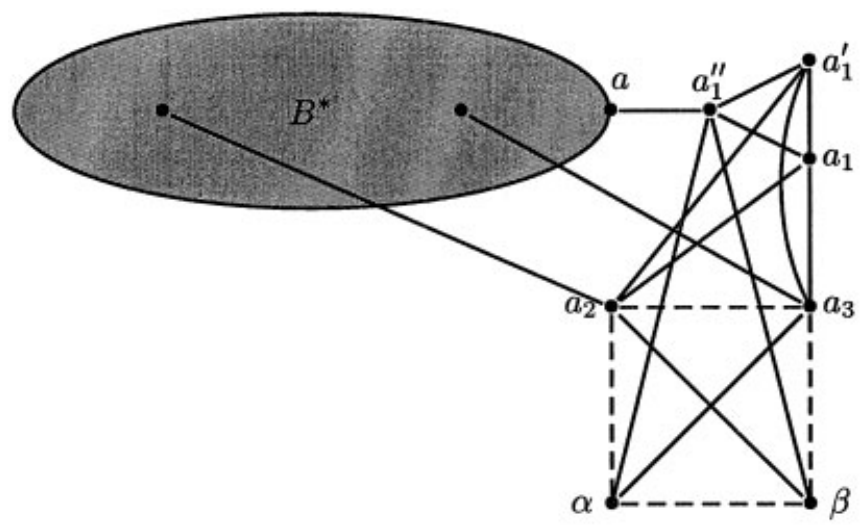

FIGURE 13. 


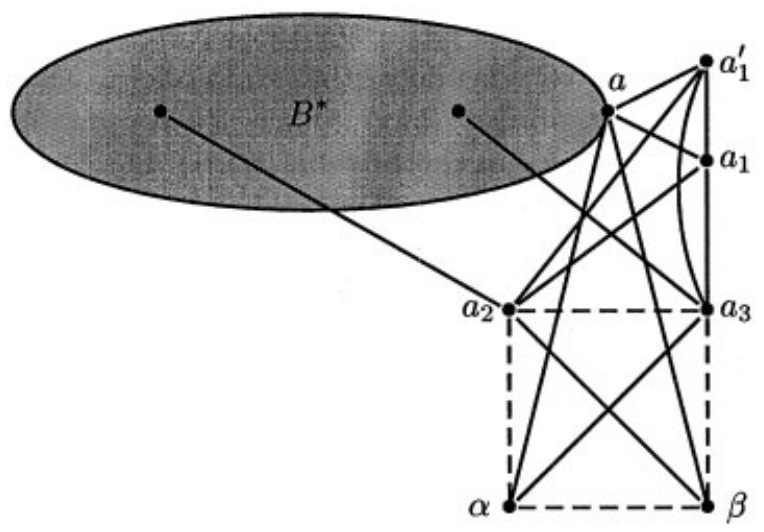

FIGURE 14.

Let $a, b$ denote the cut vertices in $G-V(C)$ of $B^{*}$. Since $G$ is 3-connected, there is a vertex $d$ of $C$ which attaches to a vertex of $B^{*}$ different from $a, b$, unless $B^{*}$ is a $K_{2}$. We shall first assume that $G-V(C)$ has an endblock, which is a $K_{2}$. Let $a_{1}$ be its vertex of degree 1 in $G-V(C)$. We can assume wlog that removal of $a$ from $G-V(C)$ disconnects $a_{1}$ from $B^{*}$. Now $a_{1}$ has three vertices of attachment in $C$, which we denote by order on $C$ as $a_{2}, a_{3}, a_{4}$. We denote by $\alpha$ the fourth vertex of $C$.

Lemma 7.1. Suppose $G-V(C)$ has two endblocks which are $K_{2}$ 's and let $b_{1}$ be the vertex of degree 1 in $G-V(C)$ of the second $K_{2}$. Suppose further that removal of a from $G-V(C)$ does not disconnect $b_{1}$ from $B^{*}$. Then $a, b$ are the only cutvertices of $G-V(C)$. The blocks of $G-V(C)$ are $B^{*}$, the two $K_{2}$ 's $a_{1} a, b_{1} b$ and triangles. The vertices of attachment in $C$ of the non-cutvertices of the triangles are precisely $a_{2}, a_{4}$.

Proof. If $b_{1}$ attaches to both $a_{3}$ and $\alpha$ then, no matter what the position of $d$ on $C$ one can can form a cycle $C^{\prime}$ of order 4 such that $G-V\left(C^{\prime}\right)$ contains a block strictly larger than $B^{*}$, contradicting (a) of Lemma 3.2. We conclude that $b_{1}$ attaches to $a_{2}$ and $a_{4}$, and that $a_{1}, b_{1}$ have exactly three vertices of attachment in $C$. Further, $d$ does not coincide with the vertex of attachment of $a_{1}, b_{1}$ different from $a_{2}, a_{4}$. In particular $d \neq a_{3}$. If $d=\alpha$ then $a_{3}$ is the third vertex of attachment in $C$ of $b_{1}$.

We show that $G-V(C)$ has no further endblocks, which are $K_{2}$ 's. Suppose for contradiction there is a third endblock which is a $K_{2}$ and its vertex of degree 1 in $G-V(C)$ is $c_{1}$. We see as in the previous paragraph that $c_{1}$ attaches to $a_{2}$ and $a_{4}$. Consider now the third vertex of attachment for $c_{1}$. If $c_{1}$ attaches to $a_{3}$ then we can form the cycle $C^{\prime}$ with edges $a_{1} a_{2}, a_{2} c_{1}, c_{1} a_{3}, a_{3} a_{1}$. If $d$ coincides with $\alpha$ or $a_{4}$ then $G-V\left(C^{\prime}\right)$ contains a block strictly larger than $B^{*}$, contradicting (a) of Lemma 3.2. If $d=a_{2}$ we also obtain a contradiction by considering the cycle $C^{\prime}$ with edges $a_{1} a_{4}, a_{4} c_{1}, c_{1} a_{3}, a_{3} a_{1}$. Suppose $c_{1}$ attaches to $\alpha$. Then by arguing as 
before we see that $d \neq a_{2}, a_{4}$ whence $d=\alpha$. In that case let $C^{\prime}$ be the cycle with edges $a_{1} a_{2}, a_{2} a_{3}, a_{3} a_{4}, a_{4} a_{1}$. Then $G-V\left(C^{\prime}\right)$ contains a block strictly larger than $B^{*}$, again a contradiction to (a) of Lemma 3.2. We conclude there are only two endblocks which are $K_{2}$ 's.

We show next that endblocks of $G-V(C)$ which are odd cycles are triangles. Further, the vertices of attachment in $C$ of the non-cutvertices of the triangle are precisely $a_{2}, a_{4}$. To see this, let $c_{1}, c_{1}^{\prime}$ be adjacent vertices of an endblock which is an odd cycle and assume they are not cutvertices of $G-V(C)$. Suppose first that $d=\alpha$, whence $a_{3}$ is the third vertex of attachment for $b_{1}$ in $C$. It is easy to see that if $c_{1}$ or $c_{1}^{\prime}$ attaches to $\alpha$ or to $a_{3}$ we will obtain a contradiction to (a) of Lemma 3.2. We conclude the vertices of attachment in $C$ of $c_{1}, c_{1}^{\prime}$ are precisely $a_{2}, a_{4}$. Suppose next that $d=a_{2}$ and $\alpha$ is the third vertex of attachment for $b_{1}$ in $C$. Assume that $c_{1}, c_{1}^{\prime}$ are connected to $B^{*}$ in $G-V(C)-\{b\}$. Then if $c_{1}$ or $c_{1}^{\prime}$ attach to $\alpha$ in $C$, we obtain a contradiction to (a) of Lemma 3.2. Hence the vertices of attachment in $C$ of $c_{1}, c_{1}^{\prime}$ can only be $a_{2}, a_{3}, a_{4}$. If $a_{3}$ is a vertex of attachment then we can form a cycle $C^{\prime}$ of order 4 with the vertices $c_{1}, c_{1}^{\prime}, a_{3}$ and one of the vertices $a_{2}$ or $a_{4}$. It is clear that $G-V\left(C^{\prime}\right)$ contains a block strictly larger than $B^{*}$, contradicting (a) of Lemma 3.2. We conclude again the vertices of attachment of $c_{1}, c_{1}^{\prime}$ in $C$ are precisely $a_{2}, a_{4}$. If $c_{1}, c_{1}^{\prime}$ are not connected to $B^{*}$ in $G-V(C)-\{b\}$, then they are connected to $B^{*}$ in $G-V(C)-\{a\}$. A similar argument implies then the vertices of attachment of $c_{1}, c_{1}^{\prime}$ in $C$ are $a_{2}, a_{4}$. Suppose finally that $d=a_{2}$ and $b_{1}$ attaches to $a_{3}$. If $c_{1}$ or $c_{1}^{\prime}$ attach to $a_{3}$ then we can argue as previously to obtain a contradiction to (a) of Lemma 3.2. Hence the vertices of attachment in $C$ of $c_{1}, c_{1}^{\prime}$ can only be $a_{2}, \alpha, a_{4}$. If $\alpha$ is a vertex of attachment, then we can form a cycle $C^{\prime}$ with vertices $c_{1}, c_{1}^{\prime}, \alpha$ and one of the vertices $a_{2}, a_{4}$. Since $G-V\left(C^{\prime}\right)$ contains a block strictly larger than $B^{*}$ we have a contradiction to (a) of Lemma 3.2. We conclude the vertices of attachment in $C$ of $c_{1}, c_{1}^{\prime}$ are precisely $a_{2}, a_{4}$. In all cases then, $c_{1}, c_{1}^{\prime}$ attach to $a_{2}, a_{4}$ in $C$. It is easy to further conclude now that the odd cycle containing $c_{1}, c_{1}^{\prime}$ as vertices is a triangle.

We show that the only cutvertices of $G-V(C)$ are $a, b$. We proceed to contradiction by assuming there are more cutvertices. Let $a^{\prime} \notin B^{*}$ be a cutvertex with the property that the connected components of $G-V(C)-\left\{a^{\prime}\right\}$ consist of the component containing $B^{*}$ and $B-\left\{a^{\prime}\right\}$ for endblocks $B$ with cutvertex $a^{\prime}$ in $G-V(C)$. We can assume wlog that $a^{\prime}$ is not the second vertex of the $K_{2}$ with vertex $b_{1}$. Suppose there is an endblock $B$ with cutvertex $a^{\prime}$ which is a triangle. Let $C^{\prime}$ be the cycle with vertices consisting of the three vertices of $B$ and the vertex $a_{2}$ or $a_{4}$ different from $d$. Then $G-V\left(C^{\prime}\right)$ contains a block strictly larger than $B^{*}$, contradicting (a) of Lemma 3.2. The alternative is that $a^{\prime}$ is the second vertex of the $K_{2}$ with vertex $a_{1}$, and $a^{\prime}$ has a vertex of attachment in $C$. Suppose that $d=\alpha$. If $a^{\prime}$ attaches to $\alpha$ then we see that if $C^{\prime}$ is the cycle with edges $a_{1} a_{2}, a_{2} a_{3}, a_{3} a_{4}, a_{4} a_{1}$ then $G-V\left(C^{\prime}\right)$ contains a block strictly larger than $B^{*}$, contradicting (a) of Lemma 3.2. Hence $a^{\prime}$ must attach to one of $a_{2}, a_{3}, a_{4}$. In that case, let $C^{\prime}$ be the cycle with vertices $a_{1}, a^{\prime}$, the vertex of attachment in $C$ of $a^{\prime}$ and an adjacent vertex not equal to $\alpha$. Then $G-V\left(C^{\prime}\right)$ contains a block strictly 
larger than $B^{*}$, again a contradiction. We conclude that $d \neq \alpha$ whence we may assume $d=a_{2}$. If $a^{\prime}$ attaches to $a_{3}, a_{4}$ or $\alpha$ we form the cycle $C^{\prime}$ with vertices $a_{1}, a^{\prime}$, the vertex of attachment in $C$ of $a^{\prime}$ and an adjacent vertex not equal to $a_{2}$. Then $G-V\left(C^{\prime}\right)$ contains a block strictly larger than $B^{*}$, contradicting (a) of Lemma 3.2. Hence $d=a_{2}$ and $a_{2}$ is the only vertex of attachment in $C$ of $a^{\prime}$.

We conclude a contradiction by showing that $a^{\prime}$ has at least two vertices of attachment in $C$. To see this first observe that $a^{\prime}$ is the cutvertex of just two blocks of $G-V(C)$, one of which is the $K_{2}, a_{1} a^{\prime}$. Further, every endblock of $G-V(C)$, which is a triangle, must have cutvertex $a$ or $b$ in $G-V(C)$. If $a^{\prime}$ has just one vertex of attachment in $C$ then the second block with cutvertex $a^{\prime}$ must be an odd cycle. Let $a^{\prime \prime}$ be a vertex of this cycle adjacent to $a^{\prime}$ which is not a cutvertex of $G-V(C)$. Then $a^{\prime \prime}$ has two vertices of attachment in $C$. Suppose $a^{\prime \prime}$ attaches to $a_{3}$. Let $C^{\prime}$ be the cycle with edges $a_{3} a_{1}, a_{1} a^{\prime}, a^{\prime} a^{\prime \prime}, a^{\prime \prime} a_{3}$. Then $G-V\left(C^{\prime}\right)$ contains a block strictly larger than $B^{*}$, contradicting (a) of Lemma 3.2. Since a similar argument can be made if $a^{\prime \prime}$ attaches to $a_{4}$, it follows that $a^{\prime \prime}$ attaches to both $a_{2}$ and $\alpha$. Now let $C^{\prime}$ be the cycle of order 6 with edges $a_{3} a_{1}, a_{1} a^{\prime}, a^{\prime} a^{\prime \prime}, a^{\prime \prime} \alpha$, $\alpha a_{4}, a_{4} a_{3}$. Then $G-V\left(C^{\prime}\right)$ contains a block strictly larger than $B^{*}$, again a contradiction. We conclude that $a^{\prime}$ has more than one vertex of attachment in $C$.

It follows now that $a, b$ are the only cutvertices of $G-V(C)$. Hence the blocks of $G-V(C)$ are $B^{*}$, the two $K_{2}$ 's $a_{1} a, b_{1} b$, and triangles with cutvertex $a$ or $b$.

Lemma 7.2. Suppose $G-V(C)$ has two endblocks which are $K_{2}$ 's and let $b_{1}$ be the vertex of degree 1 in $G-V(C)$ of the second $K_{2}$. Suppose further the removal of a from $G-V(C)$ does not disconnect $b_{1}$ from $B^{*}$. Then there is a cycle $C^{\prime}$ in $G$ of order 4 such that $G-V\left(C^{\prime}\right)$ is non-empty and connected and $G-E\left(C^{\prime}\right)$ is 2connected.

Proof. We suppose first that there is an endblock of $G-V(C)$ which is a triangle. Let $c_{1}, c_{1}^{\prime}$ be the non-cutvertices of the triangle and $a$ be the third vertex. Let $C^{\prime}$ be the cycle with edges $c_{1} a_{2}, a_{2} \alpha, \alpha a_{4}, a_{4} c_{1}$. If $b_{1}$ attaches to $a_{3}$ then $G-V\left(C^{\prime}\right)$ contains a block strictly larger than $B^{*}$, contradicting (a) of Lemma 3.2. Hence the three vertices of attachment in $C$ are $a_{2}, \alpha, a_{4}$. Let $C^{\prime}$ be the cycle with edges $c_{1} a_{2}, a_{2} c_{1}^{\prime}, c_{1}^{\prime} a_{4}, a_{4} c_{1}$. If the diagonal $a_{3} \alpha$ exists then $G-V\left(C^{\prime}\right)$ contains a block strictly larger than $B^{*}$, a contradiction. We conclude the edge $a_{3} \alpha$ does not exist. Hence $a_{3}$ has a vertex of attachment in $G-V(C)$ different from $a_{1}$. We have already seen this vertex of attachment cannot be $b_{1}$. Let $C^{\prime}$ be the cycle with edges $b_{1} a_{2}, a_{2} \alpha, \alpha a_{4}, a_{4} b_{1}$. Since $G-V\left(C^{\prime}\right)$ cannot contain a block larger than $B^{*}$, we see that the second vertex of attachment in $G-V(C)$ of $a_{3}$ must be $a$. Similarly we see that $\alpha$ attaches to $b$. The situation is illustrated in Figure 15. Let $C^{\prime}$ be the cycle with edges $a_{2} b_{1}, b_{1} a_{4}, a_{4} a_{1}, a_{1} a_{2}$. Then $G-V\left(C^{\prime}\right)$ is non-empty and connected, and $G-E\left(C^{\prime}\right)$ is 2-connected.

We may assume now that the blocks of $G-V(C)$ are $B^{*}$ and the two $K_{2}$ 's $a_{1} a, b_{1} b$. If $b_{1}$ attaches to $a_{3}$, then $\alpha$ attaches to at least two vertices of $B^{*}$, or 


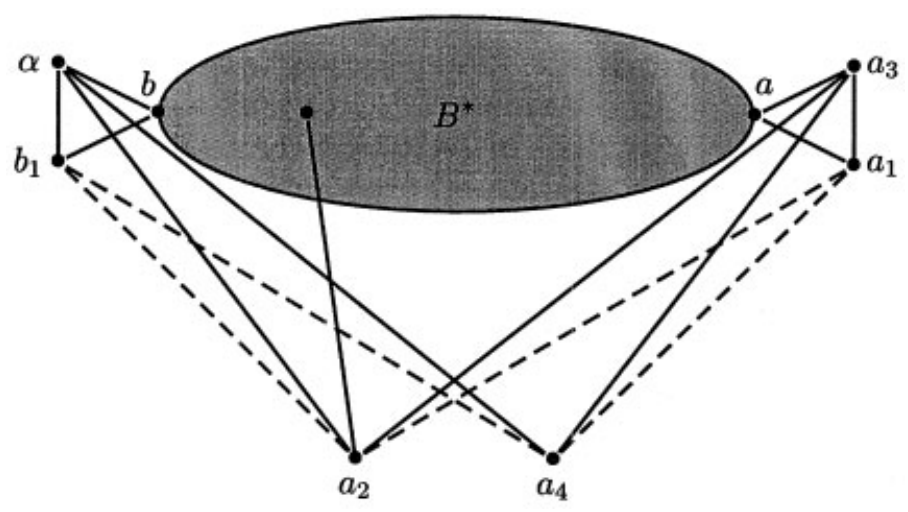

FIGURE 15.

the edge $a_{3} \alpha$ exists and $\alpha$ attaches to at least one vertex of $B^{*}$. If $\alpha$ attaches to two vertices of $B^{*}$, let $C^{\prime}$ be the cycle with edges $a_{1} a_{2}, a_{2} a_{3}, a_{3} a_{4}, a_{4} a_{1}$. Then $G-V\left(C^{\prime}\right)$ contains a block strictly larger than $B^{*}$, contradicting (a) of Lemma 3.2. If the edge $a_{3} \alpha$ exists, then $\alpha$ also attaches to a vertex of $B^{*}$. In that case it is easy to see that $G-E(C)$ is 2-connected. The situation is illustrated in Figure 16.

The final situation we need to deal with is when $b_{1}$ attaches to $\alpha$, in which case we may assume $d=a_{2}$. If the diagonal $\alpha a_{3}$ exists, then $G-E(C)$ is 2-connected so we shall assume the edge $\alpha a_{3}$ does not exist. Hence $\alpha$ has a second vertex of attachment in $G-V(C)$. This vertex of attachment must be $b$. Otherwise we can obtain a contradiction to (a) of Lemma 3.2. Similarly $a_{3}$ has a second vertex of attachment in $G-V(C)$, which must be $a$. We are in a situation similar to that illustrated in Figure 15. Hence if $C^{\prime}$ is the cycle with edges $a_{2} b_{1}, b_{1} a_{4}, a_{4} a_{1}, a_{1} a_{2}$ then $G-E\left(C^{\prime}\right)$ is 2-connected.

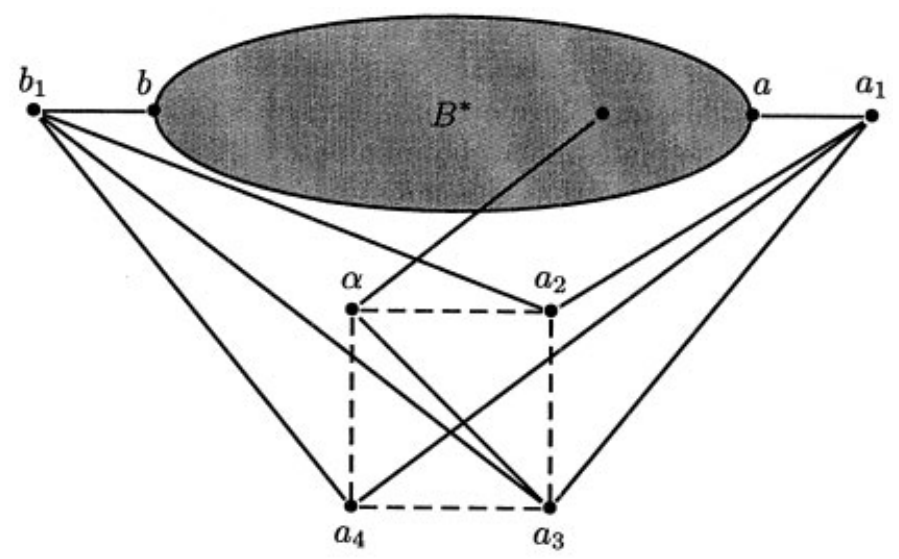

FIGURE 16. 
Next we consider the situation where all endblocks of $G-V(C)$ connected to $B^{*}$ through $b$ are odd cycles. We continue to assume that $G-V(C)$ has an endblock which is a $K_{2}$ connected to $B^{*}$ through $a$.

Lemma 7.3. Suppose $G-V(C)$ has an endblock which is a $K_{2}$, connected to $B^{*}$ through $a$, and denote by $a_{1}$ its vertex of degree 1 in $G-V(C)$. Suppose further that all endblocks of $G-V(C)$ connected to $B^{*}$ through $b$ are odd cycles. Let $b_{1}, b_{1}^{\prime}$ be adjacent vertices of such an odd cycle which are not cutvertices of $G-V(C)$. Then

(1) $a_{1}$ has three vertices of attachment in $C$, which we denote by $a_{2}, a_{3}, a_{4}$ in order on $C$,

(2) $b_{1}, b_{1}^{\prime}$ have exactly two vertices of attachment in $C$,

(3) If the vertices of attachment of $b_{1}, b_{1}^{\prime}$ are non-adjacent, on $C$ then they coincide with $a_{2}, a_{4}$.

(4) If the vertices of attachment of $b_{1}, b_{1}^{\prime}$ are adjacent on $C$, one of them must be the fourth vertex $\alpha$ of $C$.

Proof. To prove (1) assume that $a_{1}$ attaches to all four vertices of $C$. We consider the position of $d$ on $C$. If $d$ attaches to $b_{1}$ or $b_{1}^{\prime}$ then we form the cycle $C^{\prime}$ with vertices $a_{1}$ and the three vertices of $C$ different from $d$. Since $G-V\left(C^{\prime}\right)$ contains a block strictly larger than $B^{*}$, we have a contradiction to (a) of Lemma 3.2. Hence $d$ is not a vertex of attachment for $b_{1}, b_{1}^{\prime}$. In that case we can form a cycle $C^{\prime}$ of order 4 from the vertices $b_{1}, b_{1}^{\prime}$ and the three vertices of $C$ different from $d$. Again we have that $G-V\left(C^{\prime}\right)$ contains a block strictly larger than $B^{*}$, a contradiction. Hence (1) holds.

We prove (2). Assume first that there are four vertices of attachment of $b_{1}, b_{1}^{\prime}$ in $C$. If $d=\alpha$ or $d=a_{3}$ then we easily obtain a contradiction to (a) of Lemma 3.2. Hence we may assume wlog that $d=a_{2}$. One can see that it possible to form a cycle $C^{\prime}$ of order 4 from $b_{1}, b_{1}^{\prime}$ and the vertices $a_{3}, a_{4}, \alpha$ of $C$. Since $G-V\left(C^{\prime}\right)$ contains a block strictly larger than $B^{*}$, we have a contradiction to (a) of Lemma 3.2. It follows that $b_{1}, b_{1}^{\prime}$ have at most three vertices of attachment in $C$. Suppose $b_{1}, b_{1}^{\prime}$ have exactly three vertices of attachment in $C$. Hence one of these vertices attaches to both $b_{1}$ and $b_{1}^{\prime}$. Suppose the vertex is $\alpha$. If $a_{3}$ is a vertex of attachment for $b_{1}$ or $b_{1}^{\prime}$, then we may argue as in Lemma 7.1 to obtain a contradiction to (a) of Lemma 3.2. Hence we may assume that $b_{1}$ attaches to $a_{2}$ and $b_{1}^{\prime}$ to $a_{4}$. It is easy to see now that no matter where $d$ lies on $C$, we obtain a contradiction to (a) of Lemma 3.2. A similar argument can be made if the common vertex of attachment for $b_{1}$ and $b_{1}^{\prime}$ is $a_{3}$. We may therefore assume that the common vertex of attachment for $b_{1}$ and $b_{1}^{\prime}$ is $a_{2}$. Suppose now $b_{1}$ attaches to $a_{4}$, whence $b_{1}^{\prime}$ must attach to $\alpha$ or $a_{3}$. Again we see that no matter where $d$ is located on $C$, we obtain a contradiction to (a) of Lemma 3.2. Thus we may further assume that $b_{1}$ attaches to $a_{3}$ and $b_{1}^{\prime}$ to $\alpha$. In that case we must also have $d=a_{2}$. Since $\alpha$ has degree at least 4 in $G$, either the edge $\alpha a_{3}$ exists or $\alpha$ 
attaches to a vertex of $G-V(C)$ different from $b_{1}^{\prime}$. Suppose the edge $\alpha a_{3}$ exists. Let $C^{\prime}$ be the cycle with edges $a_{1} a_{3}, a_{3} \alpha, \alpha a_{4}, a_{4} a_{1}$. Then $G-V\left(C^{\prime}\right)$ contains a block strictly larger than $B^{*}$, contradicting (a) of Lemma 3.2. We conclude that $\alpha$ has a second vertex of attachment $\alpha^{\prime}$ in $G-V(C)$ and $\alpha^{\prime} \neq a_{1}, b_{1}, b_{1}^{\prime}$. Suppose $\alpha^{\prime}$ is not disconnected in $G-V(C)$ from $B^{*}$ by removal of $b$. Let $C^{\prime}$ be the cycle with edges $a_{1} a_{2}, a_{2} a_{3}, a_{3} a_{4}, a_{4} a_{1}$. Then $G-V\left(C^{\prime}\right)$ contains a block strictly larger than $B^{*}$, contradicting (a) of Lemma 3.2. The alternative is that $\alpha^{\prime}$ is not disconnected in $G-V(C)$ from $B^{*}$ by removal of $a$. In that case, let $C^{\prime}$ be the cycle with edges $a_{2} b_{1}^{\prime}, b_{1}^{\prime} b_{1}, b_{1} a_{3}, a_{3} a_{2}$. Then $G-V\left(C^{\prime}\right)$ contains a block strictly larger than $B^{*}$, contradicting (a) of Lemma 3.2. It follows that if $b_{1}, b_{1}^{\prime}$ have three vertices of attachment in $C$ we have a contradiction. Hence (2) holds.

We turn to the proof of (3) and (4). Now (3) follows by the argument of Lemma 7.1 so we consider (4). We proceed to contradiction by assuming the vertices of attachment in $C$ of $b_{1}, b_{1}^{\prime}$ are precisely $a_{2}$ and $a_{3}$. It is clear by previous argument that in this case we must have $d=a_{2}$. We show first that the only endblock of $G-V(C)$ which is disconnected from $B^{*}$ by removal of $a$ is the $K_{2}$ with vertex $a_{1}$. To see this, suppose there is a second such endblock and it is a $K_{2}$ with vertex $a_{1}^{\prime}$ of degree 1 in $G-V(C)$. Then $a_{1}^{\prime}$ has three vertices of attachment in $C$. If both the edges $a_{1}^{\prime} a_{3}$ and $a_{1}^{\prime} a_{4}$ exist, we can form the cycle $C^{\prime}$ with edges $a_{1} a_{3}, a_{3} a_{1}^{\prime}, a_{1}^{\prime} a_{4}, a_{4} a_{1}$. Since $G-V\left(C^{\prime}\right)$ contains a block strictly larger than $B^{*}$, we obtain a contradiction to (a) of Lemma 3.2. Hence the edges $\alpha a_{1}^{\prime}$ and $a_{2} a_{1}^{\prime}$ must exist. Suppose $a_{3}$ also attaches to $a_{1}^{\prime}$, whence we may form the cycle $C^{\prime}$ with edges $a_{1}^{\prime} \alpha, \alpha a_{4}, a_{4} a_{3}, a_{3} a_{1}^{\prime}$. Now $G-V\left(C^{\prime}\right)$ contains a block strictly larger than $B^{*}$, contradicting (a) of Lemma 3.2. The alternative is that $a_{4}$ attaches to $a_{1}^{\prime}$. In that case, let $C^{\prime}$ be the cycle with edges $a_{1}^{\prime} a_{2}, a_{2} \alpha, \alpha a_{4}, a_{4} a_{1}^{\prime}$. Again $G-V\left(C^{\prime}\right)$ contains a block strictly larger than $B^{*}$, yielding a contradiction. We conclude the only endblock of $G-V(C)$ which is a $K_{2}$ and is disconnected from $B^{*}$ by removal of $a$ is the $K_{2}$ with vertex $a_{1}$.

Suppose next that there is an endblock of $G-V(C)$ which is an odd cycle and is disconnected from $B^{*}$ by removal of $a$. Let $a_{1}^{\prime}, a_{1}^{\prime \prime}$ be adjacent vertices of this odd cycle, neither of which is a cutvertex of $G-V(C)$. If $a_{1}^{\prime}$ or $a_{1}^{\prime \prime}$ attaches to $a_{3}$, then let $C^{\prime}$ be the cycle with edges $a_{1} a_{2}, a_{2} \alpha, \alpha a_{4}, a_{4} a_{1}$. Since $G-V\left(C^{\prime}\right)$ contains a block strictly larger than $B^{*}$, we have a contradiction. It follows that the vertices of attachment in $C$ of $a_{1}^{\prime}, a_{1}^{\prime \prime}$ are among $a_{2}, \alpha, a_{4}$. In that case we can form a cycle $C^{\prime}$ of order 4 from the vertices $a_{1}^{\prime}, a_{1}^{\prime \prime}, a_{2}, \alpha, a_{4}$. Since $G-V\left(C^{\prime}\right)$ contains a block which includes $B^{*}$ and $a_{3}$, we have a contradiction to (a) of Lemma 3.2 again. We conclude the only endblock of $G-V(C)$ disconnected from $B^{*}$ by removal of $a$ is the $K_{2}$ with vertex $a_{1}$.

We consider next the vertices of attachment in $G-V(C)$ of $\alpha$. If the diagonal $\alpha a_{3}$ exists we can form the cycle $C^{\prime}$ with edges $a_{1} a_{3}, a_{3} \alpha, \alpha a_{4}, a_{4} a_{1}$. Since $d=a_{2}$ and $b_{1}$ also attaches to $a_{2}, G-V\left(C^{\prime}\right)$ contains a block which includes $B^{*}$ and $a_{2}$, contradicting (a) of Lemma 3.2. Hence the diagaonal $\alpha a_{3}$ does not exist, whence $\alpha$ has two vertices of attachment in $G-V(C)$. Let $\alpha^{\prime}$ be a vertex of attachment for $\alpha$ satisfying $\alpha^{\prime} \neq a$. If $\alpha^{\prime}$ is not disconnected in $G-V(C)$ from $B^{*}$ by removal 
of $a$, then we form the cycle $C^{\prime}$ with edges $b_{1} a_{2}, a_{2} a_{3}, a_{3} b_{1}^{\prime}, b_{1}^{\prime} b_{1}$. Since $G-V\left(C^{\prime}\right)$ contains a block strictly larger than $B^{*}$, we have a contradiction to (a) of Lemma 3.2. Hence we may assume $\alpha^{\prime}$ is disconnected in $G-V(C)$ from $B^{*}$ by removal of $a$. Since the only endblock of $G-V(C)$ disconnected from $B^{*}$ by removal of $a$ is the $K_{2}$ with vertex $a_{1}$, there is a path $P\left(\alpha^{\prime}, a_{1}\right)$ in $G-V(C)$ from $\alpha^{\prime}$ to $a_{1}$, which does not go through $a$. Let $C^{\prime}$ be the cycle with edges $\alpha \alpha^{\prime}$, $P\left(\alpha^{\prime}, a_{1}\right), a_{4} \alpha$, and either final edge $a_{1} a_{4}$, or final two edges $a_{1} a_{3}, a_{3} a_{4}$, whichever makes $C^{\prime}$ even. Since $d=a_{2}$, it follows that $G-V\left(C^{\prime}\right)$ contains a block which includes $B^{*}$ and $a_{2}$, contradicting (a) of Lemma 3.2. We conclude that (4) holds.

Lemma 7.4. Suppose $G-V(C)$ has an endblock which is a $K_{2}$, connected to $B^{*}$ through $a$, and denote by $a_{1}$ its vertex of degree 1 in $G-V(C)$. Suppose further that all endblocks of $G-V(C)$ connected to $B^{*}$ through $b$ are odd cycles. Let $b_{1}, b_{1}^{\prime}$ be adjacent vertices of such an odd cycle which are not cutvertices of $G-V(C)$. If the vertices of attachment in $C$ of $b_{1}, b_{1}^{\prime}$ are adjacent, then there exists a cycle $C^{\prime}$ of order 4 such that $G-V\left(C^{\prime}\right)$ is non-empty and connected, and $G-E\left(C^{\prime}\right)$ is 2-connected.

Proof. Denoting by $a_{2}, a_{3}, a_{4}$ the vertices of attachment in $C$ of $a_{1}$ and $\alpha$ the fourth vertex of $C$, we may assume by Lemma 7.3 that $b_{1}, b_{1}^{\prime}$ attach to precisely $a_{2}, \alpha$ in $C$. To avoid a contradiction to (a) of Lemma 3.2 we must also have that $d=a_{2}$. Suppose the edge $\alpha a_{3}$ exists. Then we may form the cycle $C^{\prime}$ with edges $a_{1} a_{3}, a_{3} \alpha, \alpha a_{4}, a_{4} a_{1}$. Since $G-V\left(C^{\prime}\right)$ contains a block strictly larger than $B^{*}$, we have a contradiction to (a) of Lemma 3.2. We conclude the edge $\alpha a_{3}$ does not exist, whence $a_{3}$ attaches to a vertex of $G-V(C)$ other than $a_{1}$.

We show that an endblock of $G-V(C)$, which is disconnected from $B^{*}$ by removal of $a$, is either the $K_{2}$ with vertex $a_{1}$ or a triangle with cutvertex $a$. Further, the vertices of attachment in $C$ of the non-cutvertices of the triangle are precisely $a_{2}$ and $a_{4}$. To see this, let us first suppose there is a second $K_{2}$ which is an endblock of $G-V(C)$ and is disconnected from $B^{*}$ by removal of $a$. Let $a_{1}^{\prime}$ be its vertex of degree 1 in $G-V(C)$. If $a_{1}^{\prime}$ attaches to $\alpha$ then we obtain a contradiction to (a) of Lemma 3.2 by considering the cycle $C^{\prime}$ with edges $a_{1} a_{2}, a_{2} a_{3}, a_{3} a_{4}, a_{4} a_{1}$. Hence $a_{1}^{\prime}$ attaches to $a_{2}, a_{3}, a_{4}$. We can then form the cycle $C^{\prime}$ with edges $a_{1} a_{3}, a_{3} a_{1}^{\prime}, a_{1}^{\prime} a_{4}, a_{4} a_{1}$. Since $d=a_{2}$ it follows that $G-V\left(C^{\prime}\right)$ contains a block strictly larger than $B^{*}$, contradicting (a) of Lemma 3.2. We conclude the only endblock of $G-V(C)$, which is a $K_{2}$ that is disconnected from $B^{*}$ by removal of $a$, is the $K_{2}$ with vertex $a_{1}$.

Next suppose there is an endblock of $G-V(C)$ which is an odd cycle that is disconnected from $B^{*}$ by removal of $a$. Let $a_{1}^{\prime}, a_{1}^{\prime \prime}$ be adjacent vertices of this odd cycle which are not cutvertices of $G-V(C)$. We see as in the previous paragraph that $\alpha$ is not a vertex of attachment in $C$ of $a_{1}^{\prime}, a_{1}^{\prime \prime}$. Suppose $a_{3}$ is a vertex of attachment for $a_{1}^{\prime}$. Then either $a_{2}$ or $a_{4}$ is a vertex of attachment for $a_{1}^{\prime \prime}$. Hence we can form a cycle $C^{\prime}$ with vertices $a_{1}^{\prime}, a_{1}^{\prime \prime}, a_{3}$ and one of the vertices $a_{2}, a_{4}$. It is easy 
to see that $G-V\left(C^{\prime}\right)$ contains a block strictly larger than $B^{*}$, contradicting (a) of Lemma 3.2. We conclude the vertices of attachment in $C$ of $a_{1}^{\prime}, a_{1}^{\prime \prime}$ are precisely $a_{2}, a_{4}$. It is easy to see further that the odd cycle with vertices $a_{1}^{\prime}, a_{1}^{\prime \prime}$ is a triangle. Otherwise we can obtain a contradiction to (a) of Lemma 3.2. Let $a_{1}^{\prime \prime \prime}$ be the third vertex of the triangle, whence $a_{1}^{\prime \prime \prime}$ is a cutvertex of $G-V(C)$. If $a_{1}^{\prime \prime \prime} \neq a$ we form the cycle $C^{\prime}$ with edges $a_{4} a_{1}^{\prime}, a_{1}^{\prime} a_{1}^{\prime \prime \prime}, a_{1}^{\prime \prime \prime} a_{1}^{\prime \prime}, a_{1}^{\prime \prime} a_{4}$. Since $d=a_{2}$ it follows that $G-V\left(C^{\prime}\right)$ contains a block strictly larger than $B^{*}$, contradicting (a) of Lemma 3.2. We conclude $a_{1}^{\prime \prime \prime}=a$.

It is not difficult now to see that the second vertex of the $K_{2}$ with vertex $a_{1}$, is also $a$. To see this, let $a_{1}^{\prime}$ be the second vertex and suppose $a_{1}^{\prime} \neq a$. Then, by virtue of the previous paragraph, $a_{1}^{\prime}$ is the cutvertex of exactly two blocks of $G-V(C)$. Hence $a_{1}^{\prime}$ attaches to a vertex of $C$. To avoid a contradiction to (a) of Lemma 3.2, we see that $a_{1}^{\prime}$ can attach only to $a_{2}$. Hence the second block with cutvertex $a_{1}^{\prime}$ is an odd cycle. Let $a_{1}^{\prime \prime}$ be a vertex of this odd cycle adjacent to $a_{1}^{\prime}$ which is not a cutvertex of $G-V(C)$. Then $a_{1}^{\prime \prime}$ has two vertices of attachment in $C$, whence $a_{1}^{\prime \prime}$ attaches to a vertex of $C$ other than $a_{2}$. If $a_{1}^{\prime \prime}$ attaches to $\alpha$, then we get a contradiction to (a) of Lemma 3.2 as before. If $a_{1}^{\prime \prime}$ attaches to $a_{3}$ or $a_{4}$, we can form a cycle $C^{\prime}$ with vertices $a_{1}, a_{1}^{\prime}, a_{1}^{\prime \prime}$ and fourth vertex $a_{3}$ or $a_{4}$, whichever attaches to $a_{1}^{\prime \prime}$. Since $G-V\left(C^{\prime}\right)$ contains a block strictly larger than $B^{*}$, we have a contradiction to (a) of Lemma 3.2. We conclude that the blocks of $G-V(C)$ connected to $B^{*}$ through $a$ are the $K_{2}, a_{1} a$, and triangles with cutvertex $a$. The vertices of attachment in $C$ of the non-cutvertices of the triangles are precisely $a_{2}, a_{4}$.

We consider next the blocks of $G-V(C)$ connected to $B^{*}$ through $b$. Consider first the endblock with adjacent vertices $b_{1}, b_{1}^{\prime}$ in the statement of the lemma. Now $a_{2}, \alpha$ are the vertices of attachment in $C$ of $b_{1}, b_{1}^{\prime}$ and $d=a_{2}$. By arguing as in the previous paragraph, it is easy to see that the odd cycle containing the vertices $b_{1}, b_{1}^{\prime}$ is a triangle with cutvertex $b$ in $G-V(C)$. It follows further, that any endblock of $G-V(C)$ which is an odd cycle and is connected to $B^{*}$ through $b$ is a triangle. The vertices of attachment in $C$ of the non-cutvertices of the triangle are precisely $a_{2}, \alpha$ and the cutvertex of the triangle is $b$. Otherwise we can obtain a contradiction to (a) of Lemma 3.2. We conclude the blocks of $G-V(C)$ connected to $B^{*}$ through $b$ are triangles with cutvertex $b$. The vertices of attachment in $C$ of the non-cutvertices of the triangles are $a_{2}, \alpha$.

We have already seen that the edge $\alpha a_{3}$ does not exist. In view of our analysis of the block structure of $G-V(C)$ it is easy to see then that $a_{3}$ attaches to $a \in B^{*}$. Since the vertex $a_{4}$ has degree at least 4 , either the diagonal $a_{2} a_{4}$ exists or $a_{4}$ has a vertex of attachment in $G-V(C)$ different from $a_{1}$. To avoid a contradiction to (a) of Lemma 3.2, this vertex of attachment must be $a$. Let $C^{\prime}$ be the cycle with edges $a_{2} b_{1}, b_{1} b_{1}^{\prime}, b_{1}^{\prime} \alpha, \alpha a_{2}$. Then $G-V\left(C^{\prime}\right)$ is connected and $G-E\left(C^{\prime}\right)$ is 2connected. This is illustrated in Figure 17.

Lemma 7.5. Suppose $G-V(C)$ has an endblock which is a $K_{2}$ connected to $B^{*}$ through $a$, and denote by $a_{1}$ its vertex of degree 1 in $G-V(C)$. Suppose further 


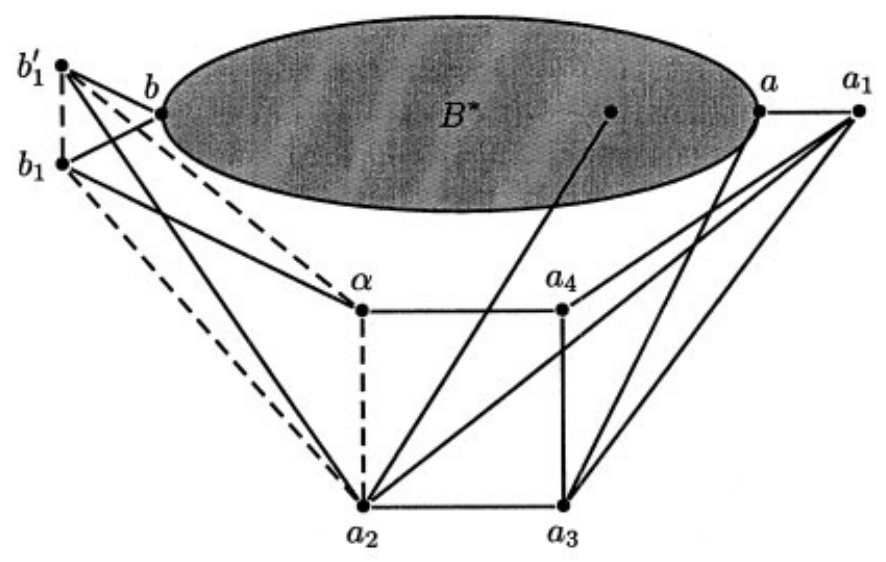

FIGURE 17.

that all endblocks of $G-V(C)$ connected to $B^{*}$ through $b$ are odd cycles. Let $b_{1}, b_{1}^{\prime}$ be adjacent vertices of such an odd cycle which are not cutvertices of $G-V(C)$. If the vertices of attachment in $C$ of $b_{1}, b_{1}^{\prime}$ are non-adjacent, then there exists a cycle $C^{\prime}$ of order 4 such that $G-V\left(C^{\prime}\right)$ is non-empty and connected, and $G-E\left(C^{\prime}\right)$ is 2-connected.

Proof. Using the notation of the previous lemmas, we see that $a_{2}, a_{4}$ are the vertices of attachment in $C$ of $b_{1}, b_{1}^{\prime}$. Suppose now $d=a_{3}$, and let $C^{\prime}$ be the cycle with edges $b_{1} a_{2}, a_{2} \alpha, \alpha a_{4}, a_{4} b_{1}$. Then $G-V\left(C^{\prime}\right)$ contains a block strictly larger than $B^{*}$, contradicting (a) of Lemma 3.2. We conclude that the only vertex of $B^{*}$ that $a_{3}$ can attach to is $a$. Suppose next $\alpha=d$. If the edge $\alpha a_{3}$ exists, we form the cycle $C^{\prime}$ with edges $b_{1} a_{2}, a_{2} b_{1}^{\prime}, b_{1}^{\prime} a_{4}, a_{4} b_{1}$. Then $G-V\left(C^{\prime}\right)$ contains a block strictly larger than $B^{*}$, contradicting (a) of Lemma 3.2. Hence we may assume that the edge $\alpha a_{3}$ does not exist, whence $\alpha$ has two vertices of attachment in $G-V(C)$. Since $b_{1}, b_{1}^{\prime}$ are not vertices of attachment for $\alpha$, we can argue as above to obtain a contradiction to (a) of Lemma 3.2. We conclude that $d \neq \alpha, a_{3}$, whence we may assume wlog $d=a_{2}$. We can show now that the edge $\alpha a_{3}$ cannot exist. If the edge did exist, we could form the cycle $C^{\prime}$ with edges $a_{1} a_{3}, a_{3} \alpha$, $\alpha a_{4}, a_{4} a_{1}$. Since $d=a_{2}$ it follows that $G-V\left(C^{\prime}\right)$ contains a block strictly larger than $B^{*}$, contradicting (a) of Lemma 3.2. We conclude $d=a_{2}$ and the edge $\alpha a_{3}$ does not exist.

We consider now the endblocks of $G-V(C)$ which are connected to $B^{*}$ through $a$. Suppose one of these is a second $K_{2}$ with vertex $a_{1}^{\prime} \neq a_{1}$ of degree 1 in $G-V(C)$. Suppose $a_{1}^{\prime}$ attaches to $a_{3}$ in $C$. Then $a_{1}^{\prime}$ attaches to $a_{2}$ or $a_{4}$. Let us suppose $a_{1}^{\prime}$ attaches to $a_{4}$ and form the cycle $C^{\prime}$ with edges $a_{1}^{\prime} a_{3}, a_{3} a_{1}, a_{1} a_{4}, a_{4} a_{1}^{\prime}$. Then $G-V\left(C^{\prime}\right)$ contains a block strictly larger than $B^{*}$, contradicting (a) of Lemma 3.2. Hence $a_{1}^{\prime}$ does not attach to $a_{4}$, whence it attaches to both $a_{2}$ and $\alpha$. Letting $C^{\prime}$ be the cycle with edges $a_{1}^{\prime} a_{3}, a_{3} a_{2}, a_{2} \alpha, \alpha a_{1}^{\prime}$, we see that $G-V\left(C^{\prime}\right)$ contains a block strictly larger than $B^{*}$, again a contradiction. We conclude 
the vertices of attachment in $C$ of $a_{1}^{\prime}$ are precisely $a_{2}, \alpha, a_{4}$. It also follows that there are at most two endblocks of $G-V(C)$ connected to $B^{*}$ through $a$ which are $K_{2}$ 's.

Consider next, an endblock of $G-V(C)$ connected to $B^{*}$ through $a$, which is an odd cycle. Let $a_{1}^{\prime}, a_{1}^{\prime \prime}$ be two adjacent vertices of the odd cycle which are not cutvertices of $G-V(C)$. We show the vertices of attachment in $C$ of $a_{1}^{\prime}, a_{1}^{\prime \prime}$ are precisely $a_{2}, a_{4}$. To see this, let us suppose first that $a_{2}$ is not a vertex of attachment for $a_{1}^{\prime}$ or $a_{1}^{\prime \prime}$. Then we can form a cycle $C^{\prime}$ of order 4 from the vertices $a_{1}^{\prime}, a_{1}^{\prime \prime}, \alpha, a_{4}, a_{3}$. Since $G-V\left(C^{\prime}\right)$ contains a block strictly larger than $B^{*}$, we have a contradiction to (a) of Lemma 3.2. We conclude $a_{2}$ is a vertex of attachment of $a_{1}^{\prime}$ or $a_{1}^{\prime \prime}$. Similarly $a_{4}$ is also a vertex of attachment for $a_{1}^{\prime}$ or $a_{1}^{\prime \prime}$. Suppose $a_{1}^{\prime}$ attaches to $\alpha$. Then $a_{1}^{\prime \prime}$ does not attach to $a_{2}$ or $a_{4}$. Otherwise we have a contradiction to (a) of Lemma 3.2. Hence $a_{1}^{\prime \prime}$ attaches to both $\alpha$ and $a_{3}$. In that case we can form the cycle $C^{\prime}$ with edges $a_{1}^{\prime \prime} a_{3}, a_{3} a_{4}, a_{4} \alpha, \alpha a_{1}^{\prime \prime}$ and $G-V\left(C^{\prime}\right)$ contains a block strictly larger than $B^{*}$. Since this contradicts (a) of Lemma 3.2, we conclude that $a_{1}^{\prime}$ does not attach to $\alpha$. By similar argument we see neither $a_{1}^{\prime}$ nor $a_{1}^{\prime \prime}$ attach to $\alpha$ or $a_{3}$, whence $a_{2}, a_{4}$ are the vertices of attachment in $C$ of $a_{1}^{\prime}, a_{1}^{\prime \prime}$.

We investigate the blocks of $G-V(C)$ which are connected to $B^{*}$ through $b$. First consider the endblock containing the vertices $b_{1}, b_{1}^{\prime}$. It is easy to see that the vertices of attachment in $C$ of any vertex of this odd cycle, which is not a cutvertex in $G-V(C)$, are precisely $a_{2}, a_{4}$. From this we conclude the odd cycle is a triangle and its cutvertex in $G-V(C)$ is $b$. Now we are assuming that any endblock of $G-V(C)$ connected to $B^{*}$ through $b$ is an odd cycle. One can see that the vertices of attachment in $C$ of the non-cutvertices of the odd cycle are precisely $a_{2}$ and $a_{4}$. Further, the odd cycle is a triangle with cutvertex $b$ in $G-V(C)$. We conclude the blocks of $G-V(C)$ connected to $B^{*}$ through $b$ are triangles with cutvertex $b$ in $G-V(C)$. The vertices of attachment in $C$ of the non-cutvertices of these triangles are precisely $a_{2}, a_{4}$.

We investigate the blocks of $G-V(C)$ which are connected to $B^{*}$ through $a$. By virtue of the previous paragraphs it is easy to see that any such endblock, which is an odd cycle, is a triangle with cutvertex $a \in B^{*}$. Next let $a_{1}^{\prime \prime}$ be a cutvertex of $G-V(C)$ with the property that $a_{1}^{\prime \prime} \notin B^{*}, a_{1}^{\prime \prime}$ is connected to $B^{*}$ through $a$, and the connected components of $G-V(C)-\left\{a_{1}^{\prime \prime}\right\}$ consist of the component containing $B^{*}$ and $B-\left\{a_{1}^{\prime \prime}\right\}$ for endblocks $B$ of $G-V(C)$. Now the only endblocks with cutvertex $a_{1}^{\prime \prime}$ are $K_{2}$ 's. Suppose $a_{1}^{\prime \prime}$ is the second vertex of two such $K_{2}$ 's, whence the edges $a_{1} a_{1}^{\prime \prime}$ and $a_{1}^{\prime} a_{1}^{\prime \prime}$ exist. Further, $a_{2}, \alpha, a_{4}$ are the vertices of attachment in $C$ of $a_{1}^{\prime}$. Let $C^{\prime}$ be the cycle with edges $a_{4} a_{1}, a_{1} a_{1}^{\prime \prime}, a_{1}^{\prime \prime} a_{1}^{\prime}, a_{1}^{\prime} a_{4}$. Then $G-V\left(C^{\prime}\right)$ contains a block strictly larger than $B^{*}$, contradicting (a) of Lemma 3.2. We conclude $a_{1}^{\prime \prime}$ is the cutvertex of exactly two blocks, one of which is an endblock that is a $K_{2}$. We shall assume this endblock is the $K_{2}$ with vertex $a_{1}$ of degree 1 in $G-V(C)$. Consider now the vertices of attachment in $C$ of $a_{1}^{\prime \prime}$. If $a_{1}^{\prime \prime}$ attaches to any of $a_{3}, a_{4}, \alpha$, we obtain a contradiction to (a) of Lemma 3.2. Hence $a_{2}$ is the only possible vertex of attachment in $C$ for $a_{1}^{\prime \prime}$. Since $a_{1}^{\prime \prime}$ is the 
cutvertex of just two endblocks, it follows that the second endblock is an odd cycle and that $a_{1}^{\prime \prime}$ attaches to $a_{2}$. We can therefore form the cycle $C^{\prime}$ with edges $a_{1} a_{3}, a_{3} a_{2}, a_{2} a_{1}^{\prime \prime}, a_{1}^{\prime \prime} a_{1}$. Now $\alpha$ has two vertices of attachment in $G-V(C)$, whence we can assume that one of these is $\alpha^{\prime} \neq b$. By the previous paragraph we see that $\alpha^{\prime}$ is not disconnected in $G-V(C)$ from $B^{*}$ by removal of $b$. It follows that $G-V\left(C^{\prime}\right)$ contains a block strictly larger than $B^{*}$, contradicting (a) of Lemma 3.2. We conclude $a_{1}^{\prime \prime}$ cannot exist. Hence the blocks of $G-V(C)$, which are connected to $B^{*}$ through $a$ are triangles with cutvertex $a$, the $K_{2}, a_{1} a$, and possibly the $K_{2}, a_{1}^{\prime} a$. The vertices of attachment in $C$ of the non-cutvertices of the triangles are precisely $a_{2}, a_{4}$. If $\alpha$ attaches to both $a$ and $b$ then we have a contradiction to (a) of Lemma 3.2. Hence the $K_{2}, a_{1}^{\prime} a$ must exist and $\alpha, a_{3}$ attach to $a$. Let $C^{\prime}$ be the cycle with edges $a_{2} a_{1}, a_{1} a_{4}, a_{4} a_{1}^{\prime}, a_{1}^{\prime} a_{2}$. Then $G-V\left(C^{\prime}\right)$ is connected and $G-E\left(C^{\prime}\right)$ is 2-connected. The situation is illustrated in Figure 18.

To complete the proof of Proposition 7.1, we need to deal with the situation where all endblocks of $G-V(C)$ are odd cycles.

Lemma 7.6. Suppose all endblocks of $G-V(C)$ are odd cycles. Let $a_{1}, a_{1}^{\prime}$ be adjacent vertices of such an odd cycle which are not cutvertices of $G-V(C)$. Then $a_{1}, a_{1}^{\prime}$ have precisely two vertices of attachment in $C$.

Proof. Suppose $a_{1}, a_{1}^{\prime}$ are connected to $B^{*}$ through $a$. Consider an endblock of $G-V(C)$ connected to $B^{*}$ through $b$. Let $b_{1}, b_{1}^{\prime}$ be adjacent vertices of $G-V(C)$. There is a vertex $d$ of $C$, which attaches to a vertex of $B^{*}$ different from $a, b$. Suppose one of $a_{1}, a_{1}^{\prime}$ attaches to $d$, but $d$ is not a vertex of attachment for $b_{1}$ or $b_{1}^{\prime}$. We can then form a cycle $C^{\prime}$ of order 4 from the vertices $b_{1}, b_{1}^{\prime}$ and

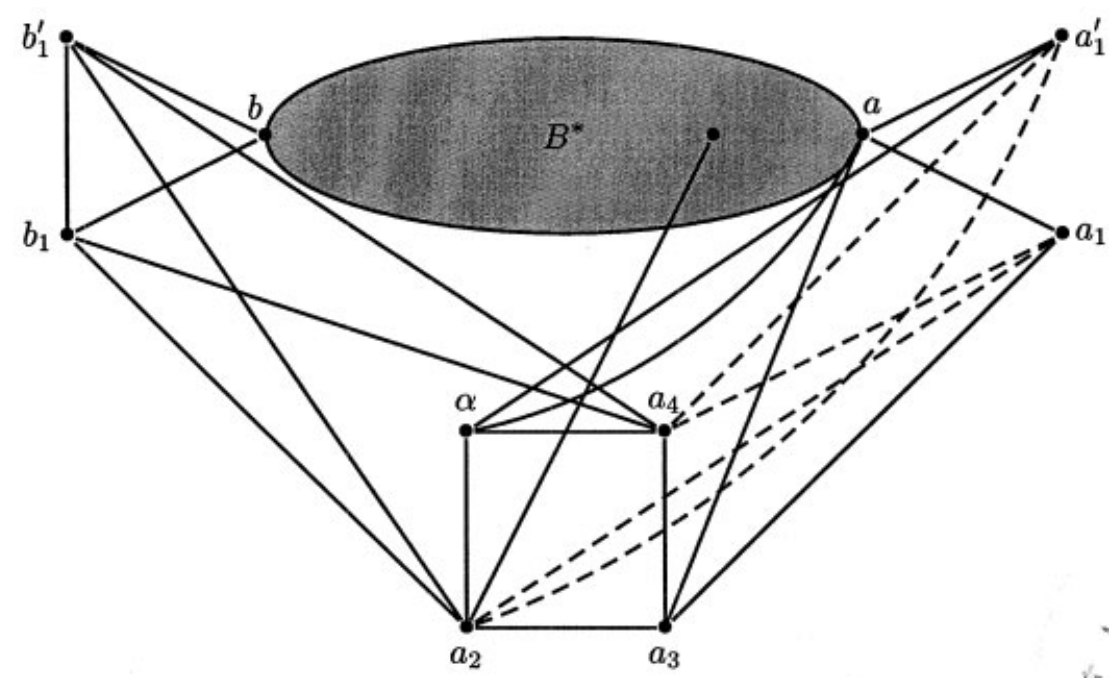

FIGURE 18. 
the three vertices of $C$ different from $d$. Since $G-V\left(C^{\prime}\right)$ contains a block strictly larger than $B^{*}$, we have a contradiction to (a) of Lemma 3.2. We conclude that there are two possibilities:

(1) One of $a_{1}, a_{1}^{\prime}$ and one of $b_{1}, b_{1}^{\prime}$ attaches to $d$,

(2) $d$ attaches to none of the vertices $a_{1}, a_{1}^{\prime}, b_{1}, b_{1}^{\prime}$.

We consider first (1). If all vertices of $C$ attach to either $a_{1}$ or $a_{1}^{\prime}$, then we can form a cycle $C^{\prime}$ of order 4 from $a_{1}, a_{1}^{\prime}$ and the three vertices of $C$ different from $d$. Since $d$ attaches to $b_{1}$ or $b_{1}^{\prime}$, it follows that $G-V\left(C^{\prime}\right)$ contains a block strictly larger than $B^{*}$, contradicting (a) of Lemma 3.2. Hence $a_{1}, a_{1}^{\prime}$ have at most three vertices of attachment in $C$, and similarly with $b_{1}, b_{1}^{\prime}$. Suppose $a_{1}, a_{1}^{\prime}$ have three vertices of attachment in $\mathrm{C}$. One of these is $d$ and we also have that $d$ is a vertex of attachment for $b_{1}$ or $b_{1}^{\prime}$. If $d$ is not a vertex of attachment for both $a_{1}$ and $a_{1}^{\prime}$, then we can form a cycle $C^{\prime}$ of order 4 from $a_{1}, a_{1}^{\prime}$ and the three vertices of $C$ different from $d$. Since $G-V\left(C^{\prime}\right)$ contains a block strictly larger than $B^{*}$, we have a contradiction to (a) of Lemma 3.2. Hence $d$ attaches to both $a_{1}$ and $a_{1}^{\prime}$. We consider the two other vertices of attachment in $C$ of $a_{1}, a_{1}^{\prime}$. It is easy to obtain a contradiction to (a) of Lemma 3.2 except in the situation where $a_{1}$ attaches to a vertex of $C$ adjacent to $d$, and $a_{1}^{\prime}$ attaches to the other vertex of $C$ adjacent to $d$. Assume now that $b_{1}, b_{1}^{\prime}$ have also three vertices of attachment in $C$. Then, by arguing as above we see that both $b_{1}$ and $b_{1}^{\prime}$ attach to $d, b_{1}$ attaches to a vertex of $C$ adjacent to $d$, and $b_{1}^{\prime}$ attaches to the other vertex of $C$ adjacent to $d$. The vertex of $C$ not adjacent to $d$ does not then attach to any of $a_{1}, a_{1}^{\prime}, b_{1}, b_{1}^{\prime}$, whence it has a vertex of attachment in $G-V(C)$ different from these. It is clear we can form a cycle $C^{\prime}$ of order 4 with vertices $d$, a vertex of $C$ adjacent to $d$, and one of the pairs $a_{1}, a_{1}^{\prime}$ or $b_{1}, b_{1}^{\prime}$ such that $G-V\left(C^{\prime}\right)$ contains a block strictly larger than $B^{*}$. We have a contradiction to (a) of Lemma 3.2. Hence $b_{1}, b_{1}^{\prime}$ must have only two vertices of attachment in $C$, one of which is $d$.

We denote the vertices of $C$ in order by $a_{2}, d, a_{3}, \alpha$. We are in the situation where $d$ is a vertex of attachment for the four vertices $a_{1}, a_{1}^{\prime}, b_{1}, b_{1}^{\prime}$, and $a_{1}$ attaches to $a_{2}$, while $a_{1}^{\prime}$ attaches to $a_{3}$. The vertices $b_{1}, b_{1}^{\prime}$ have just one other vertex of attachment in $C$. Observe that the diagonal $a_{2} a_{3}$ cannot exist. If it did, we could form the cycle $C^{\prime}$ with edges $a_{1} a_{2}, a_{2} a_{3}, a_{3} a_{1}^{\prime}, a_{1}^{\prime} a_{1}$. Since $G-V\left(C^{\prime}\right)$ contains a block strictly larger than $B^{*}$, we have a contradiction to (a) of Lemma 3.2. Suppose $\alpha$ is the second vertex of attachment in $C$ for $b_{1}, b_{1}^{\prime}$. We consider the vertex of attachment $a_{3}^{*}$ in $G-V(C)$ of $a_{3}$, which is different from $a_{1}^{\prime}, a_{1}$. If $a_{3}^{*}=a$ or is disconnected from $B^{*}$ by removal of $a$, then we form the cycle $C^{\prime}$ with edges $a_{1} a_{2}, a_{2} d, d a_{1}^{\prime}, a_{1}^{\prime} a_{1}$. In that case, $G-V\left(C^{\prime}\right)$ contains a block strictly larger than $B^{*}$, contradicting (a) of Lemma 3.2. If $a_{3}^{*} \neq a$ and is still connected to $B^{*}$ on removal of $a$, we form the cycle $C^{\prime}$ with edges $b_{1} d, d a_{2}, a_{2} \alpha, \alpha b_{1}$. Again $G-V\left(C^{\prime}\right)$ contains a block strictly larger than $B^{*}$, contradicting (a) of Lemma 3.2. We conclude $\alpha$ cannot be the second vertex of attachment in $C$ for $b_{1}, b_{1}^{\prime}$. We may therefore assume that $a_{3}$ is the second vertex 
of attachment in $C$ for $b_{1}, b_{1}^{\prime}$. In that case, $\alpha$ has a vertex of attachment $\alpha^{*}$ in $G-V(C)$ different from $a_{1}, a_{1}^{\prime}, b_{1}, b_{1}^{\prime}$. If $\alpha^{*}=a$ or is disconnected from $B^{*}$ in $G-V(C)$ by removal of $a$, we form the cycle $C^{\prime}$ with edges $a_{1} a_{2}, a_{2} d, d a_{1}^{\prime}, a_{1}^{\prime} a_{1}$. Since $G-V\left(C^{\prime}\right)$ contains a block strictly larger than $B^{*}$, we have a contradiction to (a) of Lemma 3.2. The alternative is that $\alpha^{*} \neq a$ and $\alpha^{*}$ continues to be connected to $B^{*}$ on removal of $a$ from $G-V(C)$. In that case, let $C^{\prime}$ be the cycle with edges $b_{1} d, d a_{3}, a_{3} b_{1}^{\prime}, b_{1}^{\prime} b_{1}$. Again $G-V\left(C^{\prime}\right)$ contains a block strictly larger than $B^{*}$, contradicting (a) of Lemma 3.2. We conclude that in case (1), $a_{1}, a_{1}^{\prime}$ can have only two vertices of attachment in $C$.

We turn now to case (2). Then $b_{1}$ or $b_{1}^{\prime}$ has a vertex of attachment $\beta$ in $C$ which is adjacent on $C$ to $d$. If $a_{1}, a_{1}^{\prime}$ have three vertices of attachment in $C$, we can form a cycle $C^{\prime}$ of order 4 from $a_{1}, a_{1}^{\prime}$ and the two vertices of $C$ different from $d, \beta$. Again $G-V\left(C^{\prime}\right)$ contains a block strictly larger than $B^{*}$, contradicting (a) of Lemma 3.2. We conclude then that in case (2), $a_{1}, a_{1}^{\prime}$ have just two vertices of attachment in $C$.

Lemma 7.7. Suppose all endblocks of $G-V(C)$ are odd cycles. Let $a_{1}, a_{1}^{\prime}$ be adjacent vertices of such an odd cycle which are not cutvertices of $G-V(C)$. If the vertices of attachment in $C$ of $a_{1}, a_{1}^{\prime}$ are adjacent, then $G-E(C)$ is 2connected.

Proof. We assume $a_{1}, a_{1}^{\prime}$ are connected to $B^{*}$ in $G-V(C)$ through $a$. Let $a_{2}, a_{3}$ be the vertices of attachment in $C$ of $a_{1}, a_{1}^{\prime}$, and denote by $\alpha, \beta$ the other two vertices of $C$, with $\alpha$ adjacent to $a_{2}$. We consider an endblock of $G-V(C)$ connected to $B^{*}$ through $b$. Let $b_{1}, b_{1}^{\prime}$ be adjacent vertices of this odd cycle, which are not cutvertices of $G-V(C)$. By Lemma 7.6, $b_{1}, b_{1}^{\prime}$ have two vertices of attachment in $C$. Suppose these vertices of attachment coincide with $\alpha, \beta$. Then, no matter where $d$ is located on $C$, there is a cycle $C^{\prime}$ of order 4 such that $G-V\left(C^{\prime}\right)$ contains a block strictly larger than $B^{*}$, contradicting (a) of Lemma 3.2. We have therefore that $a_{2}$ or $a_{3}$ is a vertex of attachment of $b_{1}, b_{1}^{\prime}$.

We consider the structure of endblocks of $G-V(C)$ connected to $B^{*}$ through $a$. First suppose the odd cycle containing the vertices $a_{1}, a_{1}^{\prime}$ is not a triangle, whence we may assume there is a vertex $a_{1}^{\prime \prime}$ adjacent to $a_{1}^{\prime}$, which is not a cutvertex of $G-V(C)$. Since $a_{1}^{\prime}$ attaches to $a_{2}, a_{3}$, it follows from Lemma 7.6 that $a_{2}, a_{3}$ are also the vertices of attachment in $C$ of $a_{1}^{\prime \prime}$. Hence we can form cycles $C^{\prime}$ of order 4 with vertices $a_{1}, a_{1}^{\prime}, a_{1}^{\prime \prime}$ and fourth vertex $a_{2}$ or $a_{3}$. For one of these cycles, $G-V\left(C^{\prime}\right)$ contains a block strictly larger than $B^{*}$, contradicting (a) of Lemma 3.2. We conclude the odd cycle with vertices $a_{1}, a_{1}^{\prime}$ is a triangle. It is not difficult to argue further that the cutvertex in $G-V(C)$ of the triangle is $a$. We also have that all endblocks of $G-V(C)$ connected to $B^{*}$ through $a$ are triangles with cutvertex $a$ in $G-V(C)$. Further, the vertices of attachment in $C$ of the non-cutvertices are precisely $a_{2}, a_{3}$. To see this, suppose there is a second endblock of $G-V(C)$ connected to $B^{*}$ through $a$. Let $a_{1}^{\prime \prime}, a_{1}^{\prime \prime \prime}$ be the vertices of this triangle which are not cutvertices of $G-V(C)$. If the vertices of attachment 
in $C$ of $a_{1}^{\prime \prime}, a_{1}^{\prime \prime \prime}$ are $\alpha, \beta$, then it is easy to obtain a contradiction to (a) of Lemma 3.2 by considering the position of $d$ on $C$. Let us assume that $a_{3}, \beta$ are the vertices of attachment for $a_{1}^{\prime \prime}, a_{1}^{\prime \prime \prime}$. To avoid a contradiction to (a) of Lemma 3.2, we must have $d=a_{3}$ in this case. If $a_{2}$ or $\beta$ are vertices of attachment for $b_{1}, b_{1}^{\prime}$, then we can obtain a contradiction to (a) of Lemma 3.2. It follows that $a_{3}$ and $\alpha$ are the vertices of attachment for $b_{1}, b_{1}^{\prime}$. In that case we also obtain a contradiction to (a) of Lemma 3.2. We conclude the vertices of attachment in $C$ for $a_{1}^{\prime \prime}, a_{1}^{\prime \prime \prime}$ cannot be $a_{3}, \beta$. A similar argument rules out $a_{2}, \beta$ as vertices of attachment for $a_{1}^{\prime \prime}, a_{1}^{\prime \prime \prime}$, whence $a_{2}, a_{3}$ must be the vertices of attachment.

We consider the structure of endblocks of $G-V(C)$ connected to $B^{*}$ through $b$. We see as in the previous paragraph that all such endblocks are triangles with cutvertex $b$ in $G-V(C)$. We show that the vertices of attachment in $C$ of the noncutvertices of these triangles are all identical. If the vertices of attachment in $C$ for $b_{1}, b_{1}^{\prime}$ are adjacent, we can argue exactly as in the previous paragraph to see this. Hence we can assume the vertices of attachment in $C$ for $b_{1}, b_{1}^{\prime}$ are precisely $a_{2}$ and $\beta$. To avoid a contradiction to (a) of Lemma 3.2, we must then have $d=a_{2}$. Suppose now there is a second endblock of $G-V(C)$ connected to $B^{*}$ through $b$. Let $b_{1}^{\prime \prime}, b_{1}^{\prime \prime \prime}$ be the vertices of this triangle different from $b$. We need to show that $a_{2}, \beta$ are the vertices of attachment in $C$ for $b_{1}^{\prime \prime}, b_{1}^{\prime \prime \prime}$. If $a_{3}$ is a vertex of attachment, we obtain a contradiction to (a) of Lemma 3.2. If $a_{2}$ and $\alpha$ are the vertices of attachment for $b_{1}^{\prime \prime}, b_{1}^{\prime \prime \prime}$, we can form the even cycle $C^{\prime}$ with edges $b_{1}^{\prime \prime} a_{2}, a_{2} \alpha, \alpha b_{1}^{\prime \prime \prime}, b_{1}^{\prime \prime \prime} b_{1}^{\prime \prime}$. It is clear that $G-V\left(C^{\prime}\right)$ contains a block strictly larger than $B^{*}$, contradicting (a) of Lemma 3.2. Since we can make a similar argument if $\alpha, \beta$ are the vertices of attachment for $b_{1}^{\prime \prime}, b_{1}^{\prime \prime \prime}$, we conclude the vertices of attachment for $b_{1}^{\prime \prime}, b_{1}^{\prime \prime \prime}$ must be $a_{2}, \beta$. Hence the vertices of attachment in $C$ of the non-cutvertices of the endblock triangles are all identical.

We have shown that all endblocks of $G-V(C)$ are triangles with cutvertex $a$ or $b$. The vertices of attachment in $C$ for non-cutvertices of endblock triangles connected to $B^{*}$ through $a$ are precisely $a_{2}, a_{3}$. The vertices of attachment in $C$ for non-cutvertices of endblock triangles connected to $B^{*}$ through $b$ are all identical. If $\alpha, \beta$ are these vertices of attachment, we have a contradiction to (a) of Lemma 3.2. Hence there are three possibilities:

(1) vertices of attachment are $a_{2}, a_{3}$,

(2) vertices of attachment are $a_{2}, \beta$,

(3) vertices of attachment are $a_{2}, \alpha$.

In case (1), to avoid a contradiction to (a) of Lemma 3.2, we see that the diagonals of $C, \alpha a_{3}$ and $\beta a_{2}$, must exist. Further, $\alpha, \beta$ attach to the same vertex of $B^{*}$. It is easy to see now that $G-E(C)$ is 2 -connected. This is illustrated in Figure 19.

In case (2) we obtain a contradiction to (a) of Lemma 3.2. To see this, first observe that to avoid a contradiction, $\alpha$ can have only one vertex of attachment in $G-V(C)$. Hence the diagonal $\alpha a_{3}$ exists. Suppose now $\alpha$ does not attach to $a$, 


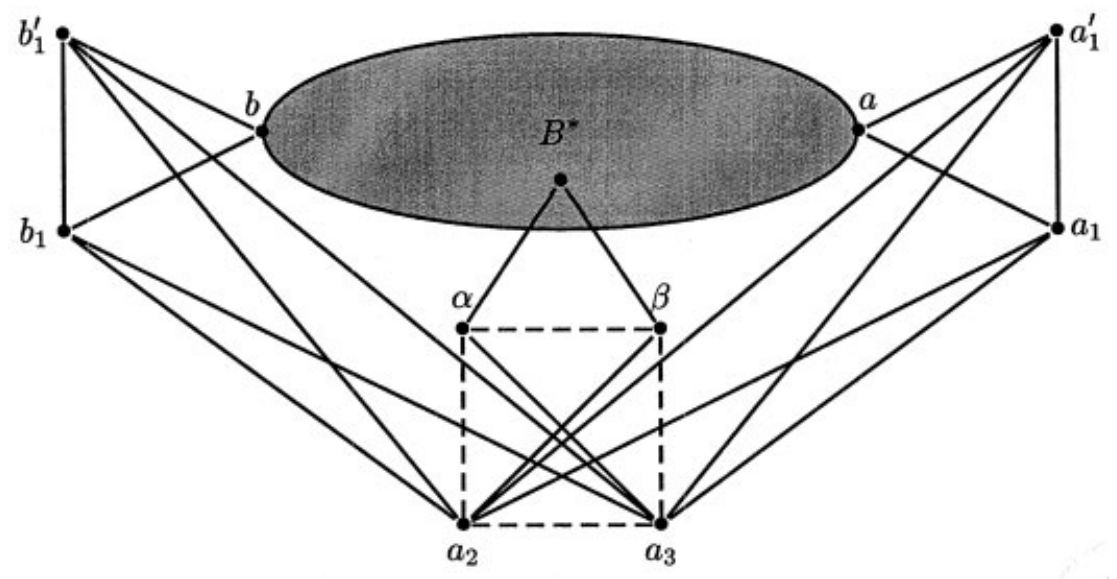

FIGURE 19.

whence it attaches to some other vertex of $B^{*}$. Let $C^{\prime}$ be the cycle with edges $b_{1} a_{2}, a_{2} b_{1}^{\prime}, b_{1}^{\prime} \beta, \beta b_{1}$. Then $G-V\left(C^{\prime}\right)$ contains a block which includes $B^{*}, \alpha$, and $a_{3}$, contradicting (a) of Lemma 3.2. Alternatively, suppose $\alpha$ attaches to $a$. If $C^{\prime}$ is the cycle with edges $a_{1} a_{2}, a_{2} a_{3}, a_{3} a_{1}^{\prime}, a_{1}^{\prime} a_{1}$, then $G-V\left(C^{\prime}\right)$ contains a block strictly larger than $B^{*}$, again a contradiction. We conclude the situation (2) is not possible.

In case (3) we also obtain a contradiction to (a) of Lemma 3.2. First observe that to avoid a contradiction, we must have $d=a_{2}$. Further, the edge $\beta a_{2}$ must exist and $\beta$ has precisely one vertex of attachment in $G-V(C)$. We can assume wlog that this vertex of attachment is $a$. Now it is clear that if $C^{\prime}$ is the cycle with edges $a_{1} a_{2}, a_{2} a_{3}, a_{3} a_{1}^{\prime}, a_{1}^{\prime} a_{1}$ then $G-V\left(C^{\prime}\right)$ contains a block larger than $B^{*}$.

Lemma 7.8. Suppose all endblocks of $G-V(C)$ are odd cycles. Let $a_{1}, a_{1}^{\prime}$ be adjacent vertices of such an odd cycle which are not cutvertices of $G-V(C)$. If the vertices of attachment in $C$ of $a_{1}, a_{1}^{\prime}$ are non-adjacent, then there is a cycle $C^{\prime}$ of order 4 such that $G-V\left(C^{\prime}\right)$ is connected and $G-E\left(C^{\prime}\right)$ is 2-connected.

Proof. We assume $a_{1}, a_{1}^{\prime}$ are connected to $B^{*}$ in $G-V(C)$ through $a$. Let $a_{2}, a_{3}$ be the vertices of attachment in $C$ of $a_{1}, a_{1}^{\prime}$, and denote by $\alpha, \beta$ the remaining two vertices of $C$. We consider an endblock of $G-V(C)$ connected to $B^{*}$ through $b$. Let $b_{1}, b_{1}^{\prime}$ be adjacent vertices of this odd cycle which are not cutvertices of $G-V(C)$. By Lemma 7.6, $b_{1}, b_{1}^{\prime}$ have two vertices of attachment in $C$. If these vertices of attachment are adjacent, then the result follows from Lemma 7.7. If $\alpha, \beta$ are the vertices of attachment for $b_{1}, b_{1}^{\prime}$, then one can obtain a contradiction to (a) of Lemma 3.2 no matter where $d$ is located on $C$. Hence we may assume $a_{2}, a_{3}$ are the vertices of attachment in $C$ for $b_{1}, b_{1}^{\prime}$. It is not difficult to see further that all endblocks of $G-V(C)$ are triangles with cutvertex $a$ or $b$. The vertices of attachment in $C$ for their non-cutvertices are precisely $a_{2}, a_{3}$. 
We consider the 2 edges with vertex $\alpha$, which do not belong to the cycle $C$. If both edges attach to vertices of $G-V(C)$-which must lie in $B^{*}$ - we have a contradiction to (a) of Lemma 3.2. Hence the diagonal $\alpha \beta$ exists and $\alpha$ has just one vertex of attachment in $G-V(C)$. Similarly $\beta$ has one vertex of attachment in $G-V\left(C^{\prime}\right)$ and it coincides with the vertex of attachment for $\alpha$. Let $C^{\prime}$ be the cycle with edges $a_{1} a_{2}, a_{2} b_{1}, b_{1} a_{3}, a_{3} a_{1}$. Then $G-V\left(C^{\prime}\right)$ is connected and $G-E\left(C^{\prime}\right)$ is 2-connected. This is illustrated in Figure 20.

Proof of Proposition 7.1. This follows from the previous lemmas.

\section{PROOF OF THEOREM 1.2-COMPLETION}

We complete the proof of Theorem 1.2 with the following.

Proposition 8.1. Suppose $G$ is a 3-connected graph of minimum degree 4 and the cycle $C$ of Lemma 3.2 has order 4 . If $B^{*}$ has at least three cutvertices in $G-V(C)$, then there is a cycle $C^{\prime}$ in $G$ of order 4 such that $G-V\left(C^{\prime}\right)$ is nonempty and connected, and $G-E\left(C^{\prime}\right)$ is 2-connected.

To begin the proof of Proposition 8.1, we establish some properties of the endblocks of $G-V(C)$.

Lemma 8.1. There are at most two cutvertices of $B^{*}$ in $G-V(C)$ with the property that there exists an endblock of $G-V(C)$ which is a $K_{2}$, connected to $B^{*}$ in $G-V(C)$ through the cutvertex.

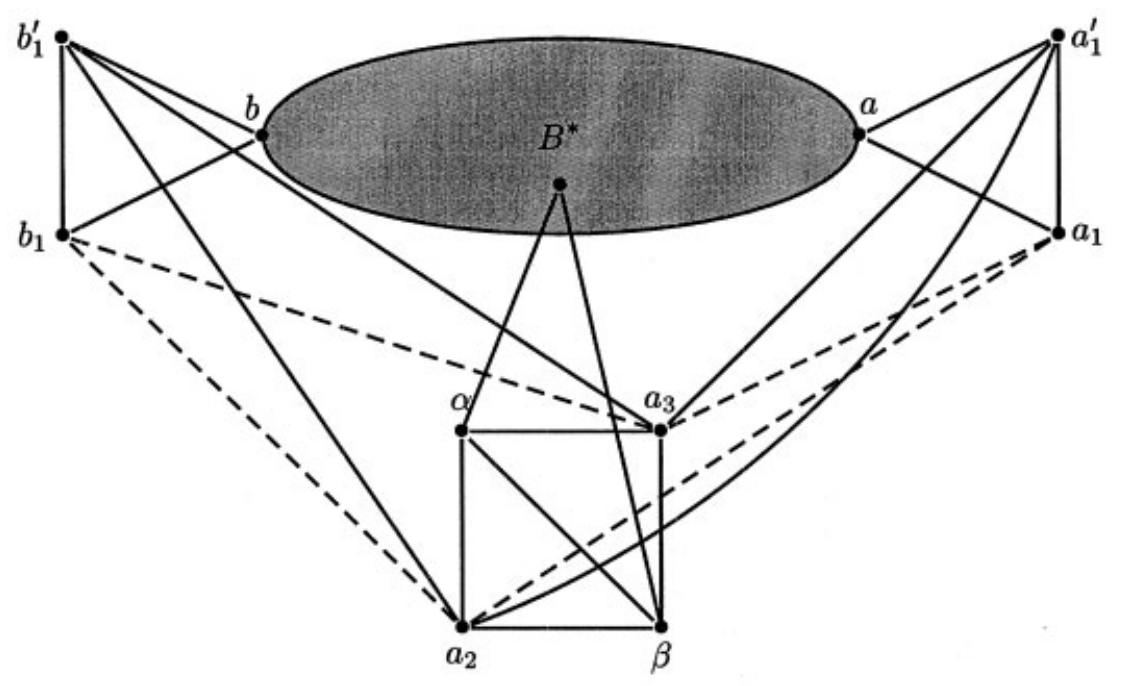

FIGURE 20. 
Proof. Suppose $B^{*}$ has cutvertices $a, b, c$ in $G-V(C)$ and that there are three endblocks which are $K_{2}$ 's with vertices $a_{1}, b_{1}, c_{1}$ of degree 1 in $G-V(C)$. Suppose further that $a_{1}$ is connected to $B^{*}$ in $G-V(C)$ through the cutvertex $a, b_{1}$ to $B^{*}$ through $b$, and $c_{1}$ to $B^{*}$ through $c$. Let $a_{2}, a_{3}, a_{4}$ be vertices of attachment for $a_{1}$ in $C$, in order on $C$, and $\alpha$ be the fourth vertex of $C$. If both $b_{1}$ and $c_{1}$ attach to $\alpha$, then the cycle $C^{\prime}$ with edges $a_{1} a_{2}, a_{2} a_{3}, a_{3} a_{4}, a_{4} a_{1}$ has the property that $G-V\left(C^{\prime}\right)$ contains a block strictly larger than $B^{*}$, contradicting (a) of Lemma 3.2. We have a similar contradiction if both $b_{1}$ and $c_{1}$ attach to $a_{3}$. Hence both $b_{1}$ and $c_{1}$ attach to $a_{2}$ and $a_{4}$, and one of $b_{1}, c_{1}$ attaches to $a_{3}$, say $c_{1}$. In that case, we again obtain a contradiction to (a) of Lemma 3.2 by considering the cycle $C^{\prime}$ with edges $b_{1} a_{2}, a_{2} \alpha, \alpha a_{4}, a_{4} b_{1}$. We conclude there are not more than two cutvertices with the property in the statement of the lemma.

Lemma 8.2. Suppose $G-V(C)$ has an endblock which is a $K_{2}$ and $a_{1}$ is its vertex of degree 1 in $G-V(C)$. Then $a_{1}$ has exactly three vertices of attachment in $C$.

Proof. Let us assume $a_{1}$ is connected to $B^{*}$ in $G-V(C)$ through the cutvertex $a \in B^{*}$ and that $a_{1}$ attaches to all four vertices of $C$. Let $b, c \in B^{*}$ be two other cutvertices of $G-V(C)$. Assume there is a $K_{2}$ which is an endblock of $G-V(C)$ and its vertex $b_{1}$ of degree 1 in $G-V(C)$ is connected to $B^{*}$ through $b$. By Lemma 8.1, all endblocks of $G-V(C)$ connected to $B^{*}$ through $c$ are odd cycles. Let $c_{1}, c_{1}^{\prime}$ be adjacent vertices of such an odd cycle, which are not cutvertices of $G-V(C)$. Now $c_{1}, c_{1}^{\prime}$ have at least two vertices of attachment in $C$, and $b_{1}$ has at least three. It follows that there is a vertex $\alpha$ of $C$ which attaches to $b_{1}$ and one of the vertices $c_{1}, c_{1}^{\prime}$. Let $C^{\prime}$ be the cycle with vertices $a_{1}$ and the three vertices of $C$ different from $\alpha$. Then $G-V\left(C^{\prime}\right)$ contains a block strictly larger than $B^{*}$, contradicting (a) of Lemma 3.2.

We conclude all endblocks of $G-V(C)$ connected to $B^{*}$ through $b$ are odd cycles. Let $b_{1}, b_{1}^{\prime}$ be adjacent vertices of such an odd cycle which are not cutvertices of $G-V(C)$. If the pairs $b_{1}, b_{1}^{\prime}$ and $c_{1}, c_{1}^{\prime}$ have a common vertex of attachment $\alpha$ in $C$, we can argue as before to obtain a contradiction to (a) of Lemma 3.2. Hence their vertices of attachment are disjoint. In that case we can form a cycle $C^{\prime}$ of order 4 with $b_{1}, b_{1}^{\prime}$, the two vertices of attachment in $C$ of $b_{1}, b_{1}^{\prime}$, and at most one other vertex of $C$. Again $G-V\left(C^{\prime}\right)$ contains a block strictly larger than $B^{*}$, contradicting (a) of Lemma 3.2.

Since in all cases we have a contradiction, we conclude that $a_{1}$ cannot have 4 vertices of attachment in $C$.

Lemma 8.3. Suppose $G-V(C)$ has an endblock which is an odd cycle, and $a_{1}, a_{1}^{\prime}$ are adjacent vertices of this cycle which are not cutvertices of $G-V(C)$. Then $a_{1}, a_{1}^{\prime}$ have exactly the same two vertices of attachment in $C$.

Proof. Suppose $a_{1}$ is connected to $B^{*}$ in $G-V(C)$ through the cutvertex $a \in B^{*}$. Let $b, c \in B^{*}$ be two other cutvertices of $G-V(C)$. Assume there is a $K_{2}$ 
with vertex $b_{1}$ of degree 1 in $G-V(C)$ which is connected to $B^{*}$ in $G-V(C)$ through $b$. Similarly assume there is a $K_{2}$ with vertex $c_{1}$ of degree 1 in $G-V(C)$ connected to $B^{*}$ in $G-V(C)$ through $c$. By Lemma 8.2, $c_{1}$ has three vertices of attachment in $C$ which we may write, in order on $C$, as $c_{2}, c_{3}, c_{4}$. Let $\alpha$ be the fourth vertex of $C$. Suppose $\alpha$ is not a vertex of attachment for $b_{1}$, whence $c_{2}, c_{3}, c_{4}$ are the vertices of attachment in $C$ for $b_{1}$. If $\alpha$ is not a vertex of attachment for $a_{1}, a_{1}^{\prime}$, then we can form a cycle $C^{\prime}$ of order 4 out of the vertices $a_{1}, a_{1}^{\prime}$, two of the vertices $c_{2}, c_{3}, c_{4}$ and $\alpha$. Since $G-V\left(C^{\prime}\right)$ contains a block strictly larger than $B^{*}$, we have a contradiction to (a) of Lemma 3.2. Hence $\alpha$ is a vertex of attachment for $a_{1}, a_{1}^{\prime}$. In that case we can form a cycle $C^{\prime}$ of order 4 out of the vertices $a_{1}, a_{1}^{\prime}, \alpha$ and at most 2 other vertices of $C$. Again $G-V\left(C^{\prime}\right)$ contains a block strictly larger than $B^{*}$, contradicting (a) of Lemma 3.2.

We conclude $\alpha$ is a vertex of attachment for $b_{1}$. Let us assume now that $c_{2}, c_{3}$ are the other vertices of attachment in $C$ for $b_{1}$. It is easy to see that if either $\alpha, c_{2}$ or $c_{4}$ is a vertex of attachment for $a_{1}, a_{1}^{\prime}$, then we can form a cycle $C^{\prime}$ such that $G-V\left(C^{\prime}\right)$ contains a block strictly larger than $B^{*}$. It follows that $c_{3}$ is the only possible vertex of attachment in $C$ for $a_{1}, a_{1}^{\prime}$. Since this again gives a contradiction, we conclude that the vertices of attachment in $C$ for $b_{1}$ are $\alpha, c_{2}, c_{4}$. We consider now the vertices of attachment in $C$ for $a_{1}, a_{1}^{\prime}$. If $\alpha$ is a vertex of attachment for $a_{1}, a_{1}^{\prime}$ then, by considering the cycle $C^{\prime}$ with edges $c_{1} c_{2}, c_{2} c_{3}, c_{3} c_{4}, c_{4} c_{1}$, we obtain a contradiction to (a) of Lemma 3.2. Making a similar argument for $c_{3}$, we conclude that $a_{1}, a_{1}^{\prime}$ have precisely two vertices of attachment in $C$, namely $c_{2}, c_{4}$.

Next we consider the situation where there is an odd cycle which is an endblock, connected to $B^{*}$ through $b$. Let $b_{1}, b_{1}^{\prime}$ be adjacent vertices of this odd cycle, which are not cutvertices of $G-V(C)$. We continue to assume the $K_{2}$ with vertex $c_{1}$ of degree 1 in $G-V(C)$, connected through $c$ to $B^{*}$, exists. Suppose $a_{1}$ or $a_{1}^{\prime}$ attach to all four vertices of $C$. There is a vertex $\beta$ of $C$ which attaches to $c_{1}$ and one of $b_{1}, b_{1}^{\prime}$. Since we can form a cycle $C^{\prime}$ of order 4 from $a_{1}, a_{1}^{\prime}$ and the three vertices of $C$ different from $\beta$, we obtain a contradiction to (a) of Lemma 3.2. We conclude $a_{1}, a_{1}^{\prime}$ have at most three vertices of attachment in $C$. Suppose there are exactly three vertices of attachment. If $\alpha$ is not one of these vertices of attachment, we can form a cycle $C^{\prime}$ of order 4 from $a_{1}, a_{1}^{\prime}$ and either of the pairs $c_{2}, c_{3}$ or $c_{3}, c_{4}$. Since one of $\alpha, c_{2}, c_{4}$ attaches to $b_{1}$ or $b_{1}^{\prime}$, we again obtain a contradiction to (a) of Lemma 3.2. Hence $\alpha$ is a vertex of attachment for $a_{1}, a_{1}^{\prime}$. Note that $\alpha$ cannot be a vertex of attachment for $b_{1}, b_{1}^{\prime}$ since we can form the cycle $C^{\prime}$ of order 4 with edges, $c_{1} c_{2}, c_{2} c_{3}, c_{3} c_{4}, c_{4} c_{1}$. Also $c_{3}$ cannot be a vertex of attachment for $b_{1}, b_{1}^{\prime}$ since we can form the cycle $C^{\prime}$ of order 4 with the vertices $a_{1}, a_{1}^{\prime}, \alpha$ and either the vertex $c_{2}$ or $c_{4}$. Hence $c_{2}$ and $c_{4}$ are the vertices of attachment in $C$ for $b_{1}, b_{1}^{\prime}$. In that case, for the cycle $C^{\prime}$ just constructed, $G-V\left(C^{\prime}\right)$ still contains a block strictly larger than $B^{*}$, contradicting (a) of Lemma 3.2. We conclude $a_{1}, a_{1}^{\prime}$ cannot have three vertices of attachment in $C$.

Finally we consider the situation where there is also an odd cycle which is an endblock, connected to $B^{*}$ through $c$. Let $c_{1}, c_{1}^{\prime}$ be adjacent vertices of this odd 
cycle, which are not cutvertices of $G-V(C)$. Suppose first that all vertices of $C$ attach to $a_{1}$ or $a_{1}^{\prime}$. If there is a vertex $\alpha$ of $C$ which attaches to one of $b_{1}, b_{1}^{\prime}$ and one of $c_{1}, c_{1}^{\prime}$, then we can form a cycle $C^{\prime}$ of order 4 from the vertices $a_{1}, a_{1}^{\prime}$ and the three vertices of $C$ different from $\alpha$. Since $G-V\left(C^{\prime}\right)$ contains a block strictly larger than $B^{*}$, we have a contradiction to (a) of Lemma 3.2. The alternative to this is that $b_{1}, b_{1}^{\prime}$ have exactly two vertices of attachment in $C$, and similarly with $c_{1}, c_{1}^{\prime}$. Further, these vertices of attachment are disjoint. It is easy to obtain a contradiction to (a) of Lemma 3.2 in this case also. We conclude that all vertices of $C$ cannot attach to $a_{1}$ or $a_{1}^{\prime}$.

Suppose now $a_{1}, a_{1}^{\prime}$ have exactly three vertices of attachment in $C$, which we denote by $a_{2}, a_{3}, a_{4}$, in order on $C$. Let $\alpha$ be the fourth vertex of $C$. Note that we can form cycles $C^{\prime}$ of order 4 from the vertices $a_{1}, a_{1}^{\prime}$ and either the pair $a_{2}, a_{3}$ or $a_{3}, a_{4}$. Since $G-V\left(C^{\prime}\right)$ cannot contain a block larger than $B^{*}$, it follows that only $a_{3}$ can be a vertex of attachment for both pairs $b_{1}, b_{1}^{\prime}$ and $c_{1}, c_{1}^{\prime}$. Suppose $\alpha$ is not a vertex of attachment for any of $b_{1}, b_{1}^{\prime}, c_{1}, c_{1}^{\prime}$. Then we may assume that $a_{2}, a_{3}$ are the vertices of attachment for $b_{1}, b_{1}^{\prime}$, and $a_{3}, a_{4}$ the vertices of attachment for $c_{1}, c_{1}^{\prime}$. In that case, let $C^{\prime}$ be the cycle with edges $b_{1} a_{2}, a_{2} a_{3}, a_{3} b_{1}^{\prime}, b_{1}^{\prime} b_{1}$. Since $G-V\left(C^{\prime}\right)$ contains a block strictly larger than $B^{*}$, we have a contradiction to (a) of Lemma 3.2. It follows that $\alpha$ is a vertex of attachment for one of $b_{1}, b_{1}^{\prime}, c_{1}, c_{1}^{\prime}$, say $b_{1}$. If $b_{1}, b_{1}^{\prime}$ have three vertices of attachment in $C$, they must be either $\alpha, a_{2}, a_{3}$ or $\alpha, a_{4}, a_{3}$. Otherwise one of $\alpha, a_{2}, a_{4}$ would be a vertex of attachment for both pairs $b_{1}, b_{1}^{\prime}$ and $c_{1}, c_{1}^{\prime}$. Assume that $\alpha, a_{2}, a_{3}$ are the vertices of attachment for $b_{1}, b_{1}^{\prime}$. Then the vertices of attachment in $C$ for $c_{1}, c_{1}^{\prime}$ must be precisely $a_{3}, a_{4}$. In that case, let $C^{\prime}$ be the cycle with edges $c_{1} a_{3}, a_{3} a_{4}, a_{4} c_{1}^{\prime}, c_{1}^{\prime} c_{1}$. Since $G-V\left(C^{\prime}\right)$ contains a block larger than $B^{*}$ we have a contradiction to (a) of Lemma 3.2. We conclude $b_{1}, b_{1}^{\prime}$ can have only two vertices of attachment in $C$, one of which is $\alpha$. Suppose $a_{3}$ is the second vertex of attachment for $b_{1}, b_{1}^{\prime}$. Then either $a_{2}$ or $a_{4}$ is a vertex of attachment for $c_{1}, c_{1}^{\prime}$, say $a_{2}$. We form the cycle $C^{\prime}$ with edges $b_{1} \alpha, \alpha a_{4}, a_{4} a_{3}, a_{3} b_{1}$. Since $G-V\left(C^{\prime}\right)$ contains a block strictly larger than $B^{*}$, we again obtain a contradiction to (a) of Lemma 3.2. We may therefore assume that $a_{2}$ is the second vertex of attachment in $C$ for $b_{1}, b_{1}^{\prime}$, whence $a_{3}, a_{4}$ must be the vertices of attachment in $C$ for $c_{1}, c_{1}^{\prime}$. By forming the cycle $C^{\prime}$ with edges $b_{1} \alpha, \alpha a_{2}, a_{2} b_{1}^{\prime}, b_{1}^{\prime} b_{1}$, we obtain a contradiction to (a) of Lemma 3.2. It follows that $a_{1}, a_{1}^{\prime}$ cannot have three vertices of attachment in $C$.

Lemma 8.4. (1) All endblocks of $G-V(C)$ which are odd cycles are triangles. (2) All cutvertices of $G-V(C)$ lie in $B^{*}$.

Proof. To see (1), we consider an endblock which is an odd cycle, and let $a_{1}, a_{1}^{\prime}$ be adjacent vertices of this cycle which are not cutvertices of $G-V(C)$. By Lemma 8.3, $a_{1}$ and $a_{1}^{\prime}$ have the same two vertices of attachment in $C$. If the cycle is not a triangle then there is a vertex $a_{1}^{\prime \prime}$ of it adjacent to $a_{1}^{\prime}$ which is also not a cutvertex of $G-V(C)$. Again by Lemma 8.3, $a_{1}^{\prime \prime}$ has the same two vertices of attachment in $C$ as $a_{1}, a_{1}^{\prime}$. If $\alpha$ is one of these vertices of attachment, we can form 
the cycle $C^{\prime}$ with edges $\alpha a_{1}, a_{1} a_{1}^{\prime}, a_{1}^{\prime} a_{1}^{\prime \prime}, a_{1}^{\prime \prime} \alpha$. Since $B^{*}$ has at least three cutvertices in $G-V(C)$, it follows that $G-V\left(C^{\prime}\right)$ contains a block strictly larger than $B^{*}$, contradicting (a) of Lemma 3.2. We conclude the endblock odd cycle containing the vertices $a_{1}, a_{1}^{\prime}$ is a triangle. It is not difficult to further conclude that the cutvertex of this triangle in $G-V(C)$ lies in $B^{*}$.

To finish the proof of (2), we need to show that any endblock of $G-V(C)$, which is a $K_{2}$, has cutvertex in $B^{*}$. Let $a_{1}^{\prime \prime} \notin B^{*}$ be a cutvertex of $G-V(C)$ with the property that the connected components of $G-V(C)-\left\{a_{1}^{\prime \prime}\right\}$ consist of the component containing $B^{*}$ and components $B-\left\{a_{1}^{\prime \prime}\right\}$ for endblocks $B$ with cutvertex $a_{1}^{\prime \prime}$ in $G-V(C)$. By the previous paragraph, the blocks $B$ must be $K_{2}$ 's. Let $a_{1}$ be the vertex of degree 1 in $G-V(C)$ of one such $K_{2}$. Suppose there is a second $K_{2}$ with vertex $a_{1}^{\prime}$ of degree 1 in $G-V(C)$. Then $a_{1}, a_{1}^{\prime}$ have a common vertex of attachment $\alpha$ in $C$. We may therefore form the cycle $C^{\prime}$ with edges $\alpha a_{1}, a_{1} a_{1}^{\prime \prime}, a_{1}^{\prime \prime} a_{1}^{\prime}, a_{1}^{\prime} \alpha$. Since $B^{*}$ has at least three cutvertices in $G-V(C)$, it follows that $G-V\left(C^{\prime}\right)$ contains a block strictly larger than $B^{*}$, contradicting (a) of Lemma 3.2. We conclude there is only one endblock of $G-V(C)$ with cutvertex $a_{1}^{\prime \prime}$, namely the $K_{2}, a_{1} a_{1}^{\prime \prime}$.

The vertex $a_{1}^{\prime \prime}$ is the cutvertex of exactly two blocks, one of which is the $K_{2}$, $a_{1} a_{1}^{\prime \prime}$. Hence $a_{1}^{\prime \prime}$ attaches to a vertex of $C$. Let $a_{2}, a_{3}, a_{4}$ be the vertices of attachment for $a_{1}$ in $C$, in order on $C$, and $\alpha$ be the fourth vertex of $C$. Suppose $a_{1}$ is connected to $B^{*}$ in $G-V(C)$ through the cutvertex $a \in B^{*}$, and $b, c \in B^{*}$ are two other cutvertices of $G-V(C)$. Let $b_{1}$ be the vertex of an endblock of $G-V(C)$, which is not a cutvertex of $G-V(C)$, and is connected to $B^{*}$ in $G-V(C)$ through $b$. Similarly, let $c_{1}$ be a corresponding vertex of an endblock connected to $B^{*}$ through $c$. Then $b_{1}$ and $c_{1}$ have a minimum of two vertices of attachment in $C$. Suppose now $a_{1}^{\prime \prime}$ attaches to $\alpha$, whence we can form cycles $C^{\prime}$ of order 4 from $a_{1}, a_{1}^{\prime \prime}$ and either of the pairs $\alpha, a_{2}$ or $\alpha, a_{4}$. Since $G-V\left(C^{\prime}\right)$ cannot contain a block strictly larger than $B^{*}$, we can assume that $\alpha, a_{2}$ are the vertices of attachment for $b_{1}$, and $\alpha, a_{4}$ the vertices of attachment for $c_{1}$. In that case, let $C^{\prime}$ be the cycle with edges $a_{1} a_{2}, a_{2} a_{3}, a_{3} a_{4}, a_{4} a_{1}$. Then $G-V\left(C^{\prime}\right)$ contains a block larger than $B^{*}$, contradicting (a) of Lemma 3.2. We conclude $a_{1}^{\prime \prime}$ does not attach to $\alpha$. A similar argument shows $a_{1}^{\prime \prime}$ does not attach to $a_{3}$.

We may assume now that $a_{1}^{\prime \prime}$ attaches to $a_{2}$. Suppose $b_{1}$ is the vertex of degree 1 in $G-V(C)$ of a $K_{2}$. Then, by the argument of Lemma 8.3, $b_{1}$ attaches to $a_{2}, \alpha, a_{4}$ and $c_{1}$ to $a_{2}, a_{4}$. Let $C^{\prime}$ be the cycle with edges $a_{2} a_{1}^{\prime \prime}, a_{1}^{\prime \prime} a_{1}, a_{1} a_{3}, a_{3} a_{2}$. Then $G-V\left(C^{\prime}\right)$ contains a block strictly larger than $B^{*}$, contradicting (a) of Lemma 3.2. It follows that $b_{1}, c_{1}$ are the vertices of endblocks which are triangles. Each of $b_{1}, c_{1}$ have two vertices of attachment in $C$. If the vertices of attachment of $b_{1}$ and $c_{1}$ are disjoint, it is easy to obtain a contradiction to (a) of Lemma 3.2. Hence $b_{1}$ and $c_{1}$ have a common vertex of attachment in $C$. If $\alpha$ is the common vertex, we form the cycle $C^{\prime}$ with edges $a_{1} a_{2}, a_{2} a_{3}, a_{3} a_{4}, a_{4} a_{1}$. Since $G-V\left(C^{\prime}\right)$ contains a block strictly larger than $B^{*}$, we have a contradiction to (a) of Lemma 3.2 again. A similar argument rules out $a_{3}$ as a common vertex of attachment for $b_{1}, c_{1}$. If $a_{4}$ is the common vertex of attachment, we form the cycle 
$C^{\prime}$ with edges $a_{2} a_{1}^{\prime \prime}, a_{1}^{\prime \prime} a_{1}, a_{1} a_{3}, a_{3} a_{2}$. Again $G-V\left(C^{\prime}\right)$ contains a block strictly larger than $B^{*}$, contradicting (a) of Lemma 3.2. Hence $a_{2}$ is the common vertex of attachment for $b_{1}, c_{1}$. Further, either $\alpha$ or $a_{3}$ must be a vertex of attachment for one of $b_{1}$ or $c_{1}$. Let us assume $b_{1}$ attaches to $\alpha$, whence $c_{1}$ attaches to $a_{3}$ or $a_{4}$. Hence if $C^{\prime}$ is the cycle with vertices $a_{2}, \alpha, b_{1}$ and the vertex of $G-V(C)$ adjacent to $b_{1}$ which is not a cutvertex of $G-V(C)$, then $G-V\left(C^{\prime}\right)$ contains a block larger than $B^{*}$, again a contradiction. Since in the other cases we also have a contradiction, we conclude $a_{1}^{\prime \prime}$ cannot exist.

Lemma 8.5. Suppose all endblocks of $G-V(C)$ are odd cycles. Then there exists a cycle $C^{\prime}$ of order 4 such that $G-V\left(C^{\prime}\right)$ is non-empty and connected, and $G-E\left(C^{\prime}\right)$ is 2-connected.

Proof. Let $a, b, c \in B^{*}$ be three cutvertices of $G-V(C)$. Then there exists an endblock of $G-V(C)$ which is a triangle and $a$ is one of its vertices. Let $a_{1}, a_{1}^{\prime}$ be the other two vertices of the triangle. Similarly there exist triangles with vertices $b, b_{1}, b_{1}^{\prime}$ and $c, c_{1}, c_{1}^{\prime}$. Let $a_{2}, a_{3}$ be the vertices of attachment in $C$ for $a_{1}, a_{1}^{\prime}$ and $\alpha, \beta$ be the remaining two vertices of $C$, with $\alpha$ adjacent to $a_{2}$. Suppose $\alpha, \beta$ are the vertices of attachment in $C$ for $b_{1}, b_{1}^{\prime}$. If $c_{1}$ attaches to $\alpha$, we form a cycle $C^{\prime}$ of order 4 out of the five vertices $a_{1}, a_{1}^{\prime}, a_{2}, a_{3}, \beta$. Since $G-V\left(C^{\prime}\right)$ contains a block strictly larger than $B^{*}$ we have a contradiction to (a) of Lemma 3.2. Arguing similarly if $c_{1}$ attaches to other vertices of $C$ we conclude that $a_{2}$ or $a_{3}$ must be a vertex of attachment in $C$ for $b_{1}, b_{1}^{\prime}$. Assume $a_{2}$ is a vertex of attachment for $b_{1}, b_{1}^{\prime}$ but $a_{3}$ is not. Suppose first that $a_{2}$ and $a_{3}$ are not adjacent, in which case we may assume wlog that $\alpha$ is the second vertex of attachment in $C$ for $b_{1}, b_{1}^{\prime}$. Now $c_{1}$ attaches to a vertex of $C$ different from $a_{2}$. If it attaches to $\alpha$, we form the cycle $C^{\prime}$ with edges $a_{1} a_{2}, a_{2} \beta, \beta a_{3}, a_{3} a_{1}$. It is clear $G-V\left(C^{\prime}\right)$ contains a block strictly larger than $B^{*}$, contradicting (a) of Lemma 3.2. If $c_{1}$ attaches to $a_{3}$ or $\beta$, we form the cycle $C^{\prime}$ with edges $b_{1} a_{2}, a_{2} \alpha, \alpha b_{1}^{\prime}, b_{1}^{\prime} b_{1}$. Again $G-V\left(C^{\prime}\right)$ contains a block larger than $B^{*}$, contradicting (a) of Lemma 3.2. We conclude that $a_{2}, a_{3}$ are adjacent. Now we need to separately consider $\alpha$ and $\beta$ as being the second vertex of attachment in $C$ for $b_{1}, b_{1}^{\prime}$. In both cases we obtain a contradiction to (a) of Lemma 3.2. It follows that $a_{3}$ is the second vertex of attachment in $C$ for $b_{1}, b_{1}^{\prime}$.

From the previous paragraph, we see that the non-cutvertices of all endblocks of $G-V(C)$ have the same two vertices of attachment $a_{2}, a_{3}$ in $C$. Suppose now $a_{2}, a_{3}$ are adjacent on $C$. If the edge $\alpha a_{3}$ does not exist, then $\alpha$ has two edges to $B^{*}$. Hence if $C^{\prime}$ is the cycle with edges $a_{1} a_{2}, a_{2} a_{3}, a_{3} a_{1}^{\prime}, a_{1}^{\prime} a_{1}$, then $G-V\left(C^{\prime}\right)$ contains a block larger than $B^{*}$, contradicting (a) of Lemma 3.2. We conclude the edge $\alpha a_{3}$ exists, and similarly the edge $\beta a_{2}$. Further, $\alpha, \beta$ must attach to the same vertex of $B^{*}$. It is easy to see now that $G-E(C)$ is 2 -connected. This has already been illustrated in Figure 19.

In the case when $a_{2}, a_{3}$ are not adjacent, then it is easy to see that the diagonal $\alpha \beta$ exists, and $\alpha, \beta$ attach to the same vertex of $B^{*}$. Let $C^{\prime}$ be the cycle with 
edges $a_{1} a_{2}, a_{2} b_{1}, b_{1} a_{3}, a_{3} a_{1}$. Then $G-V\left(C^{\prime}\right)$ is connected and $G-E\left(C^{\prime}\right)$ is $2-$ connected. This has already been illustrated in Figure 20.

Lemma 8.6. Suppose $G-V(C)$ has an endblock which is a $K_{2}$ and $a_{1}$ is its vertex of degree 1 in $G-V(C)$. Let $a_{2}, a_{3}, a_{4}$ be its vertices of attachment in $C$, in order on $C$, and $\alpha$ be the fourth vertex of $C$. Then

(1) $G-V(C)$ has exactly two endblocks which are $K_{2}$ 's. The vertices of attachment in $C$ of the non-cutvertex of the second $K_{2}$ are $a_{2}, \alpha, a_{4}$.

(2) The vertices of attachment in $C$ of a non-cutvertex of an endblock which is an odd cycle are $a_{2}, a_{4}$.

Proof. Let us suppose first that $G-V(C)$ has a second endblock which is a $K_{2}$, and $b_{1}$ is its vertex of degree 1 in $G-V(C)$. Assume further that $a_{1}$ is connected to $B^{*}$ in $G-V(C)$ through $a \in B^{*}$, and $b_{1}$ is connected to $B^{*}$ through $b \in B^{*}$ with $b \neq a$. Let $c \in B^{*}$ be a third cutvertex of $G-V(C)$. By Lemma 8.1, an endblock of $G-V(C)$ with cutvertex $c$ is an odd cycle, whence it is a triangle. Let $c_{1}$ be a non-cutvertex of this triangle. By the argument of Lemma 8.3, we see that $a_{2}, \alpha, a_{4}$ are the vertices of attachment in $C$ for $b_{1}$, and $a_{2}, a_{4}$ are the vertices of attachment for $c_{1}$. Suppose now there is another endblock which is a $K_{2}$, connected to $B^{*}$ in $G-V(C)$ through $a$. Again by the argument of Lemma 8.3, the vertices of attachment in $C$ of its non-cutvertex $a_{1}^{\prime}$ are $a_{2}, a_{3}, a_{4}$. Hence we may form the cycle $C^{\prime}$ of order 4 with edges $a_{2} a_{1}, a_{1} a_{3}, a_{3} a_{1}^{\prime}, a_{1}^{\prime} a_{2}$. Since $G-V(C)$ contains a block larger than $B^{*}$, we have a contradiction to (a) of Lemma 3.2. We conclude there are only two endblocks of $G-V(C)$ which are $K_{2}$ 's. Suppose next there is an endblock which is a triangle, connected to $B^{*}$ in $G-V(C)$ through $a$. Let $a_{1}^{\prime}, a_{1}^{\prime \prime}$ be the non-cutvertices of the triangle. If $\alpha, a_{3}$ are the vertices of attachment in $C$ for $a_{1}^{\prime}, a_{1}^{\prime \prime}$ we can form the cycle $C^{\prime}$ with edges $a_{1}^{\prime} \alpha, \alpha a_{2}, a_{2} a_{3}, a_{3} a_{1}^{\prime}$. Since $c_{1}$ and $a_{1}$ attach to $a_{4}$, we see that $G-V\left(C^{\prime}\right)$ contains a block strictly larger than $B^{*}$, contradicting (a) of Lemma 3.2. We may therefore assume $a_{2}$ is a vertex of attachment for $a_{1}^{\prime}, a_{1}^{\prime \prime}$. If $\alpha$ or $a_{3}$ is the second vertex of attachment for $a_{1}^{\prime}, a_{1}^{\prime \prime}$, we easily obtain a contradiction to (a) of Lemma 3.2. Hence $a_{2}, a_{4}$ are the vertices of attachment in $C$ for $a_{1}^{\prime}, a_{1}^{\prime \prime}$. We have proved (1) and (2) in the case when the $K_{2}$ with vertices $b_{1}$ and $b$ exists.

Suppose next that $G-V(C)$ has a second endblock which is a $K_{2}$ and $a_{1}^{\prime}$ is its vertex of degree 1 in $G-V(C)$. We assume now that both $a_{1}$ and $a_{1}^{\prime}$ are connected to $B^{*}$ in $G-V(C)$ through $a$. Let $b, c \in B^{*}$ be two other cutvertices of $G-V(C)$. From the previous paragraph, all endblocks of $G-V(C)$ with cutvertex $b$ or $c$ are triangles. Let $b_{1}, b_{1}^{\prime}$ be non-cutvertices of an endblock triangle with cutvertex $b$. Similarly, let $c_{1}, c_{1}^{\prime}$ be the corresponding vertices of a triangle with cutvertex $c$. If $\alpha$ or $a_{3}$ is a vertex of attachment for both $b_{1}$ and $c_{1}$, then we can obtain a contradiction to (a) of Lemma 3.2. Hence we may assume that $b_{1}$ attaches to $a_{2}$. If $b_{1}$ also attaches to $\alpha$, we can form the cycle $C^{\prime}$ with edges $a_{2} b_{1}, b_{1} b_{1}^{\prime}, b_{1}^{\prime} \alpha, \alpha a_{2}$. Since $c_{1}$ does not attach to $\alpha$, it must attach to $a_{3}$ or $a_{4}$. Hence $G-V\left(C^{\prime}\right)$ contains a block strictly larger than $B^{*}$, contradicting (a) of 
Lemma 3.2. It follows that $\alpha$ is not a vertex of attachment for $b_{1}$, and similarly neither is $a_{3}$. We conclude $a_{2}, a_{4}$ are the vertices of attachment in $C$ for $b_{1}$. Consider now the vertices of attachment in $C$ for $c_{1}$. If $c_{1}$ attaches to $a_{3}$, we obtain a contradiction to (a) of Lemma 3.2. Hence we may assume wlog that $c_{1}$ attaches to $a_{2}$. Arguing as before, we see that if $c_{1}$ attaches to $\alpha$ or $a_{3}$, then this gives a contradiction to (a) of Lemma 3.2. We conclude that $a_{2}, a_{4}$ are also the vertices of attachment in $C$ for $c_{1}, c_{1}^{\prime}$.

We consider the vertices of attachment in $C$ for $a_{1}^{\prime}$. Suppose $\alpha$ is not a vertex of attachment for $a_{1}^{\prime}$, whence $a_{2}, a_{3}, a_{4}$ are its vertices of attachment. We can therefore form the cycle $C^{\prime}$ with edges $a_{2} a_{1}, a_{1} a_{3}, a_{3} a_{1}^{\prime}, a_{1}^{\prime} a_{2}$, and $G-V\left(C^{\prime}\right)$ contains a block strictly larger than $B^{*}$, contradicting (a) of Lemma 3.2. Hence $a_{1}^{\prime}$ attaches to $\alpha$. If $a_{1}^{\prime}$ also attaches to $a_{3}$, we can form the cycle $C^{\prime}$ with edges $a_{1}^{\prime} \alpha, \alpha a_{2}, a_{2} a_{3}, a_{3} a_{1}^{\prime}$. In that case, $G-V\left(C^{\prime}\right)$ contains a block larger than $B^{*}$, again a contradiction to (a) of Lemma 3.2. We conclude that $a_{2}, \alpha, a_{4}$ are the vertices of attachment in $C$ for $a_{1}^{\prime}$. We can also see from the argument of Lemma 8.5 that if there is an endblock triangle with cutvertex $a$, then $a_{2}, a_{4}$ are the vertices of attachment in $C$ for its vertices different from $a$. We have proved (1) and (2) in the case when $G-V(C)$ has at least two endblocks which are $K_{2}$ 's.

We are left to deal with the case where there is just one endblock of $G-V(C)$ which is a $K_{2}$, namely the $K_{2}$ with vertices $a_{1}, a$. By the argument of the previous paragraphs, we see that $a_{2}, a_{4}$ are the vertices of attachment in $C$ for a noncutvertex of an endblock of $G-V(C)$ which is a triangle. If the edge $\alpha a_{3}$ does not exist, then $\alpha$ has two edges to $B^{*}$. Hence, if $C^{\prime}$ is the cycle with edges $a_{1} a_{2}, a_{2} a_{3}, a_{3} a_{4}, a_{4} a_{1}$, then $G-V\left(C^{\prime}\right)$ contains a block strictly larger than $B^{*}$, contradicting (a) of Lemma 3.2. If the edge $\alpha a_{3}$ does exist, then let $C^{\prime}$ be the cycle with edges $a_{1} a_{3}, a_{3} \alpha, \alpha a_{2}, a_{2} a_{1}$. Now $G-V\left(C^{\prime}\right)$ contains a block strictly larger than $B^{*}$, contradicting (a) of Lemma 3.2. We conclude $G-V(C)$ cannot have just one endblock which is a $K_{2}$.

Proof of Proposition 8.1. This follows from the previous lemmas. Note that the situation of Lemma 8.6 is similar to that illustrated in Figure 15.

\section{ACKNOWLEDGMENT}

The author thanks Andreas Blass for helpful conversations.

\section{REFERENCES}

[1] B. Bollobas, Modern Graph Theory, Springer, New York, 1998, MR 99h:05001.

[2] J. Bondy, Basic graph theory: Paths and circuits, Handbook of Combinatorics, Vol. 1, R. Graham, M. Grotschel, and L. Lovasz, Editors, Elsevier, Amsterdam, 1995, pp. 3-110. MR 97a:05129. 
[3] J. Bondy and A. Vince, Cycles in a graph whose lengths differ by one or two, J Graph Theory 27 (1998), 11-15. MR 98m:05107.

[4] A. Frank, Connectivity and network flows, Handbook of Combinatorics, Vol. 1, R. Graham, M. Grotschel, L. Lovasz, Editors, Elsevier, Amsterdam, 1995, pp. 111-177. MR 97i:05084.

[5] B. Jackson, Removable cycles in 2-connected graphs of minimum degree at least 4, J Lond Math Soc 21 (1980), 385-392. MR 81h:05089.

[6] M. Lemos and J. Oxley, On removable circuits in graphs and matroids, J Graph Theory 30 (1999), 51-66. MR 99m:05094.

[7] W. Mader, Kreuzungfreie a,b-Wege in endlichen Graphen, Abh Math Sem Univ Hamburg 42 (1974), 187-204. MR 54\#2525.

[8] C. Thomassen and B. Toft, Non-separating induced cycles in graphs, J Combin Theory Ser B 31 (1981), 199-224. MR 82m:05062.

[9] W. Tutte, Graph Theory, With a foreword by J. A. Nash-Williams, Reprint of the 1984 original, Encyclopedia of Mathematics and its Applications, 21, Cambridge University Press, Cambridge, 2001. MR 1813436.

[10] H. Voss, Cycles and bridges in graphs, Mathematics and its Applications, 49, Kluwer, Berlin, 1991. MR 92m:05118. 